\title{
Mass Spectrometry for Multimodal Imaging of Lipids in Brain Tissue
}

Citation for published version (APA):

Škrášková, K. (2016). Mass Spectrometry for Multimodal Imaging of Lipids in Brain Tissue. [Doctoral Thesis, Maastricht University]. Maastricht University. https://doi.org/10.26481/dis.20160303ks

Document status and date:

Published: 01/01/2016

DOI:

10.26481/dis.20160303ks

Document Version:

Publisher's PDF, also known as Version of record

\section{Please check the document version of this publication:}

- A submitted manuscript is the version of the article upon submission and before peer-review. There can be important differences between the submitted version and the official published version of record.

People interested in the research are advised to contact the author for the final version of the publication, or visit the DOI to the publisher's website.

- The final author version and the galley proof are versions of the publication after peer review.

- The final published version features the final layout of the paper including the volume, issue and page numbers.

Link to publication

\footnotetext{
General rights rights.

- You may freely distribute the URL identifying the publication in the public portal. please follow below link for the End User Agreement:

www.umlib.nl/taverne-license

Take down policy

If you believe that this document breaches copyright please contact us at:

repository@maastrichtuniversity.nl

providing details and we will investigate your claim.
}

Copyright and moral rights for the publications made accessible in the public portal are retained by the authors and/or other copyright owners and it is a condition of accessing publications that users recognise and abide by the legal requirements associated with these

- Users may download and print one copy of any publication from the public portal for the purpose of private study or research.

- You may not further distribute the material or use it for any profit-making activity or commercial gain

If the publication is distributed under the terms of Article $25 \mathrm{fa}$ of the Dutch Copyright Act, indicated by the "Taverne" license above, 


\section{Mass Spectrometry for \\ Multimodal Imaging of Lipids in \\ Brain Tissue}

Karolina Škrášková 
ISBN/EAN: 978-90-824913-0-2

$\mathrm{PhD}$ thesis Maastricht University

Mass Spectrometry for Multimodal Imaging of Lipids in Brain Tissue

Copyright $@ 2016$ by Karolina Škrášková, Maastricht, The Netherlands

The research reported in this dissertation was carried out at:

FOM-Institute AMOLF, Science Park 104, 1098 XG, Amsterdam, The Netherlands; and

Maastricht MultiModal Molecular Imaging Institute (M4I), Universiteitssingel 50, 6229 ER, Maastricht, The Netherlands.

A digital version of this thesis can be downloaded from www.amolf.nl. Paper copies are available through the library of FOM-Institute AMOLF and the library of Maastricht University. 


\title{
Mass Spectrometry for Multimodal Imaging of Lipids in Brain Tissue
}

Massaspectrometrie voor multimodale beeldvorming van lipiden in hersenenweefsel

\section{DISSERTATION}

to obtain the degree of Doctor at Maastricht University, on the authority of the Rector Magnificus, Prof. dr. L. L. G. Soete in accordance with the decision of the Board of Deans, to be defended in public on Thursday $3^{\text {rd }}$ March 2016 at 10.00 am by

\author{
Karolina Škrášková \\ born on $6^{\text {th }}$ January 1987 \\ in Plzen, Czech Republic
}




\section{Supervisor}

Prof. dr. Ron M. A. Heeren

\section{Co-supervisor}

Dr. Shane R. Ellis

\section{Assessment Committee}

Prof. dr. Steven W. M. Olde Damink (Chairman)

Dr. Maarten A. F. Altelaar (Utrecht University)

Prof. dr. Garry L. Corthals (University of Amsterdam)

Prof. dr. Tilman M. Hackeng

Prof. dr. Peter J. Peters

This work was supported by "Stichting voor Fundamenteel Onderzoek der Materie (FOM)", which is financially supported by the "Nederlandse organisatie voor Wetenschappelijk Onderzoek (NWO)". Further funding was provided by the Dutch national program COMMIT and NWO in the framework of the Technology Area COAST. Additionally, this research has been made possible with the support of the Dutch Province of Limburg. Finally, part of the research was supported by FP7 European Union Marie Curie IAPP Program, BRAINPATH. 
Ipse se nihil scire id unum sciat.

- Socrates 



\section{Contents}

1. Prologue: Of Brains and Molecules ...................................................................... 10

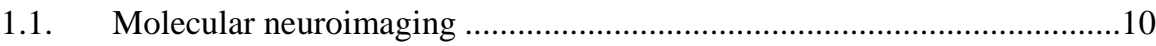

1.2. Mass spectrometry imaging .................................................................11

1.3. Brain lipidome and the role of MSI....................................................13

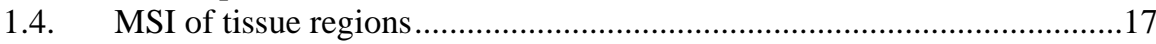

1.5. Scope of the thesis ..............................................................................18

2. Combination of matrix-assisted laser desorption ionization mass spectrometry imaging and separation techniques: A review ................................... 22

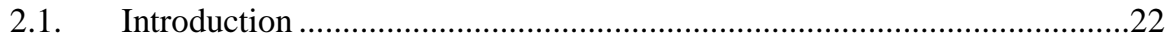

2.2. Separations for effective sample clean-up ..............................................25

2.2.1. Sample supports for MALDI-MS .........................................................25

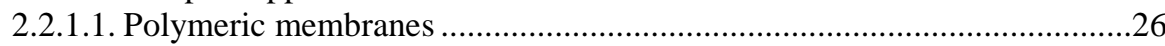

2.2.1.2. MALDI sample supports with a modified surface .....................................27

2.2.2. Washing protocols for MALDI-MSI of tissue samples...............................28

2.3. Separations within defined spatial dimensions ..........................................29

2.3.1. Planar layout: one- and two-dimensional separations ................................29

2.3.1.1. Gel electrophoresis ................................................................................

2.3.1.2. Thin layer chromatography …………………………..............................

2.3.2. Blotting: adding the $\mathrm{z}$-dimension ……………………………………......

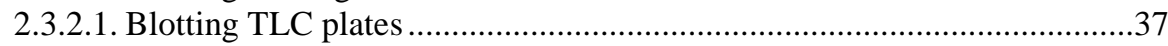

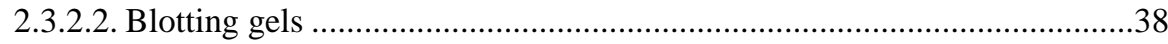

2.3.2.3. Blotting tissue sections ..............................................................................

2.4. One step blotting \& digestion..................................................................40

2.4.1. Protein identification strategies for MS .....................................................4

2.4.2. Molecular scanner ................................................................................. 41

2.5. Column and capillary separations .........................................................42

2.5.1. Direct MALDI interface .......................................................................42

2.5.2. Validation and corroboration of MSI results .............................................44

2.6. Conclusions and future perspectives ........................................................45

3. Desorption electrospray ionization and MALDI mass spectrometry imaging coupled to ion mobility separation: Enhanced lipid information ............................ 48

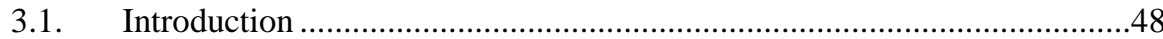

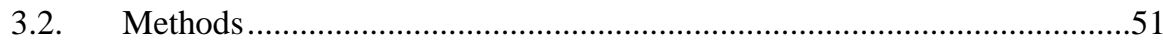

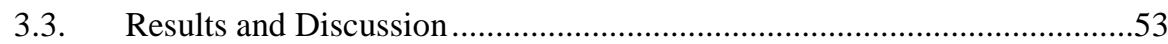

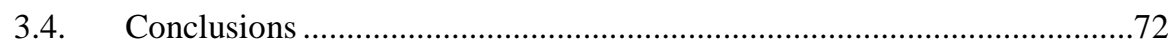


4. Fiducial markers for combined secondary ion mass spectrometry and matrix-assisted laser desorption ionization imaging...................................................74

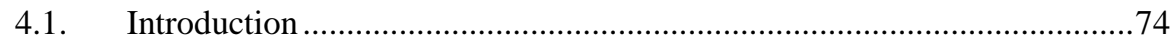

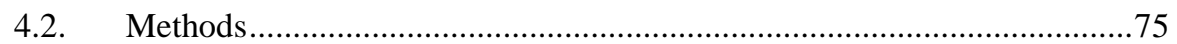

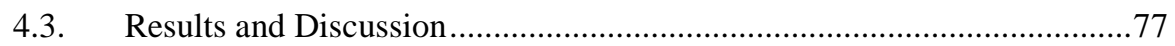

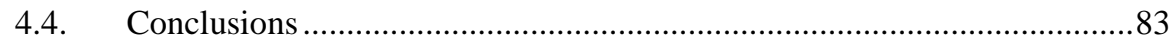

5. Co-registration of secondary ion mass spectrometry images and the Allen Brain Atlas for precise anatomic localization of accumulated lipids ......................86

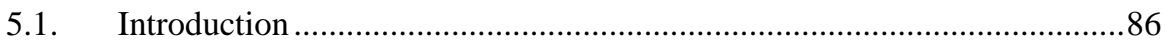

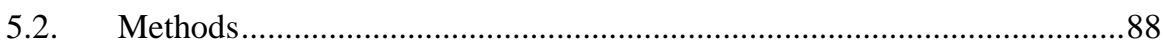

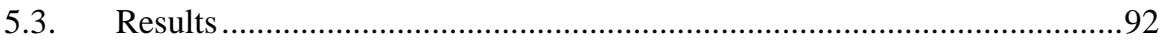

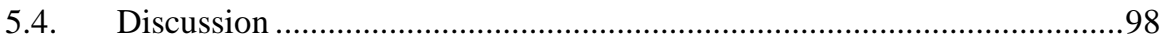

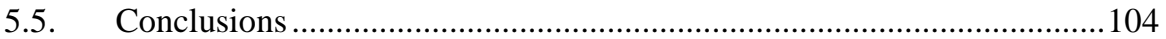

6. Epilogue: The Dynamic Knowledge .................................................................106

6.1. General discussion .........................................................................106

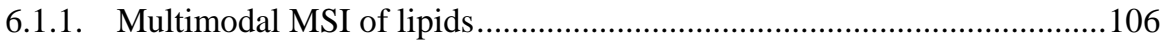

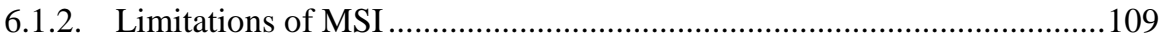

6.2. Summary ....................................................................................

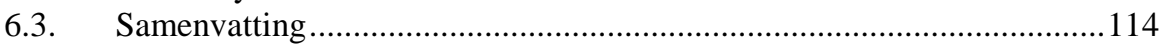

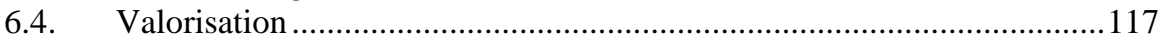

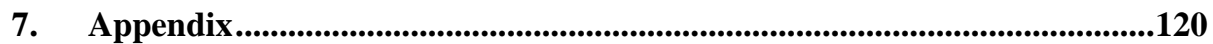

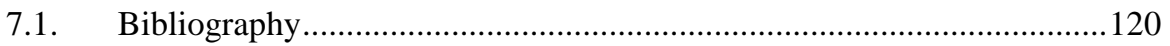

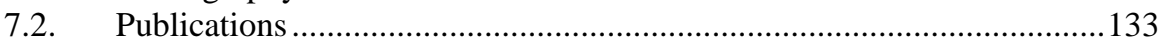

Acknowledgements ...............................................................................................................135

Curriculum Vitae.....................................................................................................................139 


\section{Prologue: Of Brains and Molecules}




\section{Prologue: Of Brains and Molecules}

No organ has ever attracted more philosophic attention than the brain. In the course of times, many philosophers (or scientists) expressed countless beliefs and views on what the brain is, how it looks like and how it works. In ancient Greece, Aristotle (384 - 322 BC) assumed that the brain's role is to cool down the heart - the organ of thought and sensation, as he believed. In Aristotle's view the organ and the basis of thought were not the same though. The latter, he pondered, was immaterial and could not be found anywhere within a body. Born centuries later, Rene Descartes (1596 - 1650) was also a dualist. Considering the brain the organ responsible for thinking, he likened its function to a hydraulic pump coordinating the movement of the fluids within. However, he assumed that this mechanism could not account for some of the higher mental faculties such as intellect and emotion. Similarly to Aristotle, Descartes believed it was the mind, or the soul, if you like, and not the brain, containing our thoughts, desires and emotions.

Nowadays, physicalism has taken over the metaphysics and we generally understand the brain as an extremely efficient computer, which operates and controls our entire bodies. Gone, or at least left aside, are the ponderings about the immaterial soul. Instead, brain research revolves around the brain's infinitesimal, nevertheless, genuinely material parts: the cells and the ever smaller molecules. Brain researchers define their aims clearly: to describe, to understand and to mimic the brain's very functioning.

But it is not only brain function, that we try to understand so desperately. Brain dysfunction is researched with similar enthusiasm. The desire to understand what happens in the brain during a malady, how the cells' behaviour changes, and which molecules are involved in it, is the driving force behind the research of brain pathophysiology. Equipped with modern analytical techniques, we can now explore the brain in detail greater than neither Aristotle nor Descartes would have ever dreamt of. We must bear in mind, however, that our knowledge of the brain remains vastly overshadowed by what we don't know. Just like Socrates pondered: The more we learn, the less we know.

\subsection{Molecular neuroimaging}

We can proclaim neuroimaging as the flagship of brain research without any exaggeration. Neuroimaging can visually describe both, the brain function and its structure. A historic experiment performed by an Italian physiologist Angelo Mosso (1846 - 1910) and known as the "human circulation balance" can be considered an ancestor of modern functional neuroimaging [1]. During his experiments, Mosso lay volunteers down on a long wooden plank similar to a seesaw, which he had carefully 
balanced. As Mosso described in his publications, when he distracted his volunteers by, for instance, ringing a bell, the front of the plank outweighed its rear end. The brain, as Mosso explained, had to process the sound, requiring more blood and thus increasing its weight [2]. Although being based on more sophisticated principles, functional magnetic resonance imaging (fMRI) and positron emission tomography (PET) are routinely used in clinical practice to monitor blood flow and metabolic activity of the brain in vivo [3].

The brain can be also explored ex vivo. Santiago Ramón y Cajal (1852 - 1934) and Camillo Golgi (1843 - 1926) are known for their ex vivo investigation of the brain structure [4]. The two Nobel laureates described brain morphology, highlighted by a (back then revolutionary) staining protocol applied to brain tissue sections. Nowadays, a number of different histochemical protocols are employed to image distinct structural and chemical details of the brain in ex vivo experiments.

Functional and structural neuroimaging are sometimes integrated under the term molecular neuroimaging. It refers to a simultaneous localization and identification of molecules present in the brain and involved in its processes. The brain is one of the most complex matters in the Universe. Many types of molecules, ranging from low mass metabolites to high mass proteins and protein complexes, are present in the brain. The molecules have diverse roles and are crucial for the functioning of the brain and thus the entire body. Various biochemical processes are dependent on mutual molecular interactions and dynamic spatial re-distribution of these molecules [5]. Moreover, properties and organization of the molecules within the brain further project into its cells, the cellular systems of the entire body, and further still into our behaviour and cognition. Hence, their knowledge becomes of special importance on the way to the understanding of the brain.

The challenge in state-of-the-art molecular neuroimaging is to achieve high molecular specificity at high spatial resolution. In other words, we want to know exactly what is exactly where. A novel molecular imaging technique called mass spectrometry imaging (MSI) addresses these questions with particular success and forms the base of this thesis. Its principles are briefly introduced in the following section.

\subsection{Mass spectrometry imaging}

Since its first introduction by J. J. Thompson (1856 - 1940) more than a hundred years ago [6], mass spectrometry (MS) has been revolutionizing the field of analytical chemistry. Moreover, this unique analytical tool has united physics, chemistry and biology under one roof. Several Nobel prizes were awarded for the MS-based scientific discoveries, proving MS an outstanding analytical technique. 
A mass spectrometer can be described as the world's smallest scales. It has the capability to determine the molecular masses of atoms and molecules - ranging from single atoms to large biomolecules - present in complex samples. Structure of the molecules can be further determined through their fragmentation patterns produced during tandem MS experiments. Fundamental research of MS enabled improvements in many of its aspects, including sensitivity, mass resolution and mass range. Introduction of the so called soft ionization techniques - matrix-assisted laser desorption ionization [7] and electrospray ionization [8] - was a turning point especially for the latter. These techniques enabled MS analysis of large biomolecules such as proteins and also meant improvement in MS analysis of lipids. Imaging of biological tissue samples based on MS, is one of its latest major methodological developments [9]. Owing to its fast progress during the past twenty years, MSI has moved from the margins to the centre of MS attention.

During a MSI experiment, a sample surface (e.g. a thin brain section) is probed at discrete positions, which are (on average) tens of micrometers apart. A mass spectrum is collected at each of the sampling locations. Molecules present at the probed positions are desorbed from the sample surface, ionized and the ions are further separated in a mass analyzer based on their mass-to-charge ratio $(\mathrm{m} / \mathrm{z})$. The final MS image is defined by the sampling coordinates and by the intensities of a particular $\mathrm{m} / \mathrm{z}$ value visualized in a colour range. We can reconstruct such an image for every $\mathrm{m} / \mathrm{z}$ present in the collected spectra. As a result, we create a molecular map of the sample surface. Two further features make MSI unique among molecular imaging techniques: 1. MSI does not require pre-experimental labelling and can therefore be used without a priori knowledge of a sample's molecular composition. 2 . MSI can image on a micrometer scale not only one, but hundreds of molecules from a single tissue section and in one experiment. Moreover, these molecules can be identified based on the general principles of (tandem) MS.

A basic MSI workflow is depicted in Figure 1.1. Sample preparation for MSI typically consists of tissue collection and sectioning, which are optionally followed by a treatment of the tissue surface: washing steps, application of matrix or on-tissue digestion protocols can be required based on the types of molecules targeted in a specific MSI study. In principle, sample preparation can vary from a close-to-none to a lengthy procedure. Collection of data is automated. Its duration is dependent on the sample size and spatial resolution employed. MSI data acquisition can take from a couple of hours to a full day. The size of MSI datasets typically increases proportionally to the duration of an acquisition and to other parameters such as employed mass resolution or mass range. To alleviate the data load advantage is taken of various strategies for data processing and further of automated data mining approaches $[10,11]$. In principle, pre-processing of MSI and conventional MS data 
does not differ [10]. The aim is to clean spectra from baseline and noise and to select peaks that encode relevant information (a step referred to as "peak picking"). Multivariate data analysis is often employed for further data exploration. To illustrate, component methods (e.g. principal component analysis, probabilistic latent semantic analysis etc.) are employed for reducing data dimensionality [12-14], clustering algorithms (e.g. hierarchical clustering, k-means clustering, etc.) are used for spatial segmentation [15-17] and methods such as linear discriminant analysis are used for tissue molecular classification [18].

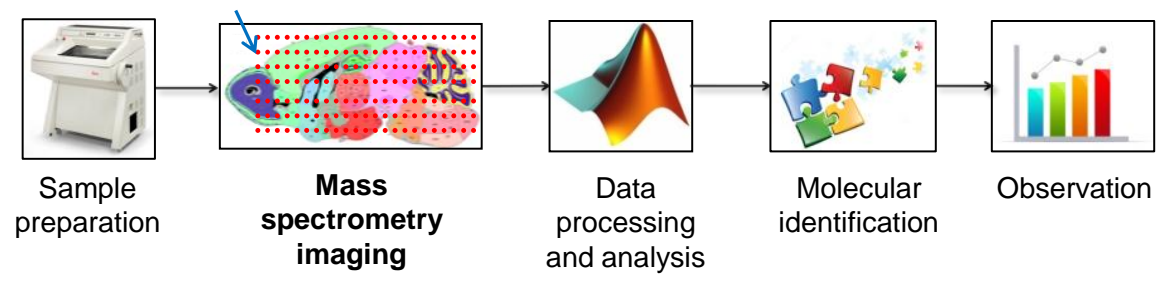

Figure 1.1 A general diagram of the MSI analytical approach. Sample preparation and collection of data are followed by data processing and multivariate data analysis. The latter typically leads to a selection of molecules with (biological) relevance. Importantly, their identification is assessed through tandem mass spectrometry experiments. Finally, conclusions are made based on the observations.

\subsection{Brain lipidome and the role of MSI}

The brain is our "fattest" organ, since more than half of its content is created by lipids [3]. Lipids include a wide range of molecules with an extremely broad chemical composition [19]. Based on their structure, lipids are divided into eight main categories: fatty acyls, glycerolipids, glycerophospholipids, sphingolipids, saccharolipids, polyketides, sterol lipids and prenol lipids. Each of the category further includes many classes and subclasses of lipids [20, 21].

The enormous variation in lipid structure reflects the vast diversity of their functions: they play structural, energetic and signalling roles within the brain and the whole body. For example, triacylglycerols are the major form of energy storage in mammals and are virtually not present in the brain. Glycerophospholipids and cholesterol are the main structural components of biological membranes [20]. Lipids, such as polyunsaturated fatty acids or prostaglandins also contribute to cell communication while taking part in various signalling pathways, thus influencing for example neural plasticity, brain repair and neuroprotection [22].

On one hand, changes in lipid metabolism can significantly influence brain function. For example, lysosomal storage disorders such as Gaucher disease [23] or Niemann-Pick disease [24] are characterized by a missing or dysfunctional enzyme 
which is responsible for degradation of particular lipid classes. Upon such a defect, lipids accumulate in different organs of the patients, including the brain. The symptoms typically include impaired functions of the central nervous system or swelling of the affected organs. On the other hand, many brain pathologies, including neurodegenerative $[25,26]$ and psychiatric disorders [27, 28] and injuries of the central nervous system [29], have been shown to be accompanied by qualitative, quantitative or spatial alterations of the lipids within the brain.

Understanding the brain lipidome is of the utmost importance should we want to know how the brain works or what is happening when something goes wrong. However, chemical analysis of lipids becomes very challenging due to their high molecular complexity and a wide array of lipid species present in the brain and in biological samples in general $[30,31]$. A typical lipidomic study involves extraction of the lipids from a tissue and a subsequent MS and tandem MS analysis of the extracts (chromatographic separation step can be employed) [32]. However, extraction and tissue homogenization lead to a loss of the spatial information, i.e. the information about the position of the lipids within a tissue and about their spatial relationship. This disadvantage can be partly overcome by region selective extraction. For example, instead of total brain lipid extracts, forebrain or cerebellum can be targeted specifically [26]. However, such a sample preparation becomes very lengthy and impractical. Almeida et al. recently published a method for effective in situ microextraction of lipids from tissue surface. A novel surface sampling technique called pressurized liquid extraction surface analysis combined with nano-electrospray ionization and high mass resolution MS enabled quantitative analysis of lipids at $400 \mu \mathrm{m}$ spatial resolution directly from a brain tissue section [33].

Conventional methods for simultaneous detection and localization (i.e. imaging) of lipids within the brain and other tissue types include histochemical imaging techniques combined with optical microscopy. These include histological staining and immunohistochemistry [34]. While they provide a (sub-)cellular spatial resolution, these methods suffer from a lack of specificity, the unavailability of antibodies or impossibility of using multiple tags in one experiment. To demonstrate, histochemical staining of lipids (such as Oil Red O [35] or Nile Red staining [36]) stains all neutral lipids or all phospholipids, respectively. Also other imaging techniques, such as magnetic resonance spectroscopy imaging (MRSI), suffer from low molecular specificity and also relatively low spatial resolution. For example, MRSI provides a signal for all choline containing compounds (total choline), to which choline, phosphocholine, glycerophosphocholines and sphingomyelines contribute simultaneously [37].

MSI, on the contrary, offers a unique opportunity to explore the brain lipidome at the micrometer scale and to determine the chemical structure of particular lipid 
species detected from specific tissue locations. The molecular structure of the lipid species is typically assessed during tandem MS experiments (typically through collision induced dissociation). Thus, MSI can image for example a phosphocholine with a known total number of fatty acyl carbons and double bonds. However, it has to be noted that it remains blind to the position of the fatty acyls, position of double bonds within a carbon chain and to its potential branching. Practical examples of lipid identification during MSI are presented in Chapter 3 of this thesis. Importantly, MSI can image multiple lipid species in one experiment. MSI can thus significantly contribute to the molecular understanding of the brain and to monitor lipidome changes related to its altered functions.

MSI is generally described as a label-free molecular imaging technique. However, the ionization efficiency of particular molecules (lipids) depends highly on the employed ionization technique, sample preparation or an experimental setup. (And, as explained in Chapter 2, also on the chemical composition of the analysed area.) The three most routinely employed ionization techniques for MSI are matrix-assisted laser desorption ionization (MALDI), desorption electrospray ionization (DESI), and secondary ion mass spectrometry (SIMS). The fundamental difference between these ionization techniques dwells in the character of the beam probing a sample surface. In MALDI, the beam is composed of a stream of photons, in DESI of a stream of charged solvent droplets, and in SIMS the beam is created by primary ions. The character of the beam has an impact on the type of analyzable molecules (i.e. mass range) and on the achievable spatial resolution. All of the aforementioned techniques were employed in the experiments presented in this thesis. Their more detailed description is provided in the respective chapters of this thesis as follows: MALDI Chapter 2 and Chapter 4, DESI Chapter 3, SIMS Chapter 4 and Chapter 5. A concise overview of the characteristics of each of the MSI technique discussed in this thesis is included in Table 1.1.

SIMS, DESI and MALDI are commonly used for lipid imaging. MALDI is most frequently employed for MSI of phospholipids and some of the ceramide based lipids such as sulphatides and sphingomyelines [38-41]. These lipid classes ionize well also in DESI [42, 43]. When compared to MALDI, DESI is especially beneficial for detection of fatty acyls and low mass lipids due to lack of matrix background [44] and importantly, as shown in Chapter 3 of this thesis, for detection of fragile lipid species such as gangliosides. SIMS images fatty acyls, cholesterol and glycerolipids particularly well [45]. The latter, however, with a significant degree of fragmentation. Fragmentation of lipids in SIMS is decreased by the use of cluster primary ion beams. For example Debois et al. detected a number of intact glycerolipids from liver tissue using bismuth $\left(\mathrm{Bi}_{3}{ }^{+}\right)$cluster ions [46]. Similarly Sjovall et al. reported on detection of intact glycerophospholipids and sulphatides from a murine brain tissue section using 
golden $\left(\mathrm{Au}_{3}^{+}\right)$cluster ions [47]. Table 1.1 further summarizes lipid substrates and other analytes frequently imaged with SIMS, DESI and MALDI.

$\begin{array}{llll}\text { Ionization } & \begin{array}{l}\text { Probing } \\ \text { beam }\end{array} & \begin{array}{l}\text { Spatial } \\ \text { resolution }\end{array} & \text { Lipid substrates }\end{array}{ }^{\text {a) }} \quad$ Other analytes

Primary ions

SIMS $\begin{aligned} & \text { (atoms or } \\ & \text { clustered } \\ & \text { atoms) }\end{aligned} \quad$ Below $1 \mu \mathrm{m} \quad \begin{aligned} & \text { Fatty acyls, cholesterol, } \\ & \text { glycerolipids }{ }^{\text {b) }} \text { [48] }\end{aligned} \quad$ Elements [49]

Fatty acyls,

glycerophospholipids, sphingolipids: sulphatides, Metabolites [50] sphingomyelines $[42,43]$, fragile lipid species: gangliosides ${ }^{\text {c) }}$

MALDI $\begin{array}{ll}\text { Laser } \\ \text { (photons) } \quad 10^{1} \mu \mathrm{m}\end{array}$

Glycerophospholipids, sphingolipids: sulphatides, sphingomyelines $[51,52]^{\text {d) }}$
Metabolites, peptides, proteins $[18,53,54]$

Table 1.1 A concise overview of mass spectrometry ionization techniques presented in this thesis and their characteristics. Listed are lipid substrates and other analytes which are typically imaged with the respective MSI techniques. Note that the ionization of particular molecules further depends on a sample preparation and an experimental setup. This is further discussed in Chapter 3 of this thesis.

a) Note that the table is not intended as a comprehensive summary of all lipid classes ever detected using SIMS, DESI and MALDI imaging. Instead, it summarizes lipids frequently imaged with the respective MSI ionization techniques.

b) Detection of intact glycerolipids is enhanced by the use of cluster ion beams such as $\mathrm{Bi}_{3}{ }^{+}$[46]. Similarly, SIMS imaging of intact glycerophospholipids was reported when cluster ion beam was employed in the experiments [47].

c) See Chapter 3 of this thesis.

d) Also MALDI imaging of glycerolipids from kidney tissue sections was reported using a specialized sample preparation based on silver nanoparticles [55]. Using specific sample preparation, also gangliosides were imaged by MALDI [56] - this is further discussed in Chapter 3 of this thesis. 
MSI of murine brain tissue has been reported multiple times. A single brain section typically features many anatomical areas with different molecular composition. That makes brain tissue practical for development of data analysis approaches such as spatial segmentation based on spatially aware clustering [15]. Improvements of sample preparation and MSI instrumental development are also frequently reported using murine brain tissue. For example: novel matrices for MALDI imaging [40], washing protocols for enhanced imaging of lipids [57] or improvements in instrumentation [13, 58]. From biomedical perspective, MSI was used to explore lipidome changes in brain after chronic alcohol administration [39] or following experimentally induced cerebral ischemia [38]. Applications can be found in Alzheimer's disease [45, 59, 60] or neuropsychiatric disorders [61] research. Moreover, molecular exploration of the brain and other tissue types using MSI is not limited to lipids but targets other molecular species. Examples of such applications can be found in literature [5, 62-64].

\subsection{MSI of tissue regions}

Biological structures, and above all the brain, are extremely complex samples. Classification and molecular distinction of specific anatomic and functional brain regions becomes crucial in understanding of the brain function or the mechanisms of brain pathologies. MSI is capable of telling apart distinct tissue regions even if those are not differentiated by any of the established histological and histochemical methods. The classification of the regions is based solely on their MS profile [65].

In a MSI approach, assessment of a particular tissue region can be approached by different strategies: 1. Performance of MS profiling / imaging at low spatial resolution to designate the regions with variable MS profiles. 2. Employment of histochemical protocols and other imaging modalities to assign the regions of interest based on the structural features. 3. Making use of a multivariate data analysis that are consecutively applied on the acquired MSI datasets. In the following paragraphs, I will briefly discuss these three approaches.

\section{- Increasing spatial resolution}

According to the mode of mass spectra acquisition and the spatial resolution employed MS profiling and MS imaging are distinguished. In the latter, a sample surface is probed in a predefined raster pattern and a mass spectrum is collected at each position. Profiling on the contrary involves mass spectra acquisition from discrete spots on the tissue surface. Only a few spots within a tissue surface are typically probed during MS profiling experiments. Profiling allows for a quick comparison of different tissue types or different tissue regions without the need for a time-consuming MSI of the whole tissue section. Profiling experiments are usually 
fast, easy to perform, less complex with decreased requirements on data analysis, and allow for a fast survey of the molecular content of distinct tissue regions. After regions with variable molecular signature are recognized, they can be MS imaged for a more detailed spatial-molecular information [66, 67]. Alternatively, pilot experiments can be performed in imaging mode at low spatial resolution.

- Histology driven MSI

Histochemical methods are often used hand in hand with MS profiling or imaging of tissue regions. Molecularly unspecific dyes, such as Nissl stain (Cresyl violet) or immunohistochemistry tags, mark different cell types or tissue regions with similar histology [34]. The following MSI analysis can be consequently aimed solely at the highlighted areas. In the histology driven MSI approach it is possible to either use an adjacent tissue section, or perform both / all experiments on the same sample.

- Data extraction

This approach is histology-independent and is based on analysis of the whole imaging dataset typically by the means of unsupervised variance analysis. There is no need for an a priori histological annotation. One of the possible approaches of data extraction, widely respected in the MSI community, is based on regional tissue segmentation performed, for instance, by clustering the mass spectra. Such a segmentation map described for example by Alexandrov et al. [15, 16] highlights spatial regions with a similar chemical composition. We have employed a similar approach in Chapter 5 of this thesis, where principal component analysis highlighted a particular region within the brain and assisted in uncovering its molecular composition.

\subsection{Scope of the thesis}

The brain is a lipid dense organ. Lipids play structural as well as signalling roles within the brain and are also involved in some of the brain pathophysiological states. MSI allows for a detailed molecular investigation of the brain lipidome. In this thesis I describe novel methodologies for MSI of lipids in the brain tissue. The methods have the potential to be combined in a comprehensive workflow that can be further employed in the molecular investigation of the brain tissue.

In Chapter 2: Mass Spectrometry Imaging and Complementary Separations, general principles of MSI are described in more detail, with a special emphasis on matrix-assisted laser desorption ionization (MALDI) imaging. The chapter reviews various examples of MALDI-MSI combined with different separation methods. These include electrophoresis, chromatography and blotting. We stress that only the 
combination of the separation techniques and MSI leads to comprehensive local sample composition information.

Chapter 3: Ambient Mass Spectrometry Imaging and Ion Mobility Separation, describes combination of MSI and ion mobility separation (IMS). In addition to MALDI, we introduce another MS ionization technique: desorption electrospray ionization (DESI). In this study we show, that DESI enables spatial analysis of fragile lipid classes. Using a simple protocol, we detected gangliosides and their acetylated versions directly from the murine brain tissue and imaged some of them. The unique combination of DESI with IMS simplified data interpretation by separating gangliosides from the rest of the detected lipid classes based on their charge state. Our novel approach advances the in situ analysis of gangliosides. In addition, we compare DESI imaging with MALDI imaging experiments performed on the same ion mobility enabled instrument. We show that by combining different MSI modalities, we can gather complementary lipid information.

In Chapter 4: Fiducial Markers for Multimodal Mass Spectrometry Imaging, we broaden the repertoire of the commonly used MSI modalities with another MS ionization technique, namely secondary ion mass spectrometry (SIMS). In this chapter we describe a proof-of-principle of a novel co-registration method for multimodal MSI including SIMS and MALDI. We sputtered gold fiducial markers next to a tissue section and imaged them together with the tissue using SIMS and MALDI subsequently. The markers were clearly visible in images acquired with both modalities, and ultimately enabled their co-registration.

Chapter 5: The Allen Brain Atlas, describes a practical use of a pipeline for an automated co-registration of SIMS acquired brain images with the Allen Brain Atlas (ABA). Using SIMS imaging we found fatty acids rich deposits located in specific regions of murine brains with impaired lipid metabolism. The employment of the workflow described in this chapter enabled precise anatomic localization of the lipidrich hotspot. We present a novel approach for the spatial characterization of regionspecific molecular imprints provided by MSI.

A fusion of the novel protocols described in this thesis led to a proposal of a comprehensive workflow which can be applied in further molecular investigation of the brain, and partly other tissue types. The workflow, its suggested methodological improvements and involvement in future research are described and discussed in Chapter 6: Epilogue: The Dynamic Knowledge. 



\section{Mass Spectrometry Imaging and Complementary Separations}

Based on: Karolina Škrášková and Ron M. A. Heeren: A review of complementary separation methods and Matrix assisted laser desorption ionization-mass spectrometry imaging: lowering sample complexity. J. Chromatogr. A 1319, 1-13 (2013). 


\title{
2. Combination of matrix-assisted laser desorption ionization mass spectrometry imaging and separation techniques: A review
}

\begin{abstract}
Matrix-assisted laser desorption ionization (MALDI) mass spectrometry imaging (MSI) brings unique combined information on molecular identity and molecular distribution of a sample surface. During the past decade, it has matured and is now routinely employed for biomedical tissue sections analysis. However, owing to the high molecular complexity of tissue, MALDIMSI suffers from ionization suppression effects. This directly results in a reduced ability to detect low-abundant molecular species. At the same time, the spatial resolution of separation techniques is often insufficient for an unambiguous determination of the local composition of a mixture. As analytical separation techniques can significantly reduce ionization suppression, and MALDI-MSI has an ability to improve their spatial resolution, the two analytical approaches can be successfully combined in a pursuit of comprehensive local sample composition information. In the following review we summarize strategies of mutually beneficial combinations of MALDI-MSI and different separation techniques and discuss limitations and future developments.
\end{abstract}

\subsection{Introduction}

Matrix-assisted laser desorption ionization (MALDI) introduced in 1985 by Karas et al. [7] pushed the borders of laser desorption ionization (LDI) techniques forward by enabling ionization of large (bio)polymers. Ultimately the ability to analyze intact macromolecules led to the emergence of new analytical disciplines such as proteomics and metabolomics. The groundbreaking improvement was based on the use of matrix. Typically a small organic compound, the matrix has a triple impact on the desorption-ionization process. (i) It separates individual molecules of the analyte from each other, thus minimizing analyte aggregation. (ii) Matrix molecules absorb the initial energy of a laser pulse. As a consequence, the molecules of analyte do not undergo direct photon induced fragmentation and organic compounds up to several tens to hundreds of kilo Daltons can be analyzed. (iii) It promotes ion-molecule reactions in the gas phase (MALDI plume), that results in formation of pseudomolecular ions from analyte molecules [68-70].

The most common sample preparation for a MALDI experiment is the dry droplet method: a solution of analyte(s) is mixed with a matrix solution and small droplets of the mixture are spotted onto a metal target. Upon the droplet drying, cocrystals of matrix and analyte are formed. The actual desorption-ionization process is 
initiated by exposure to one or more laser pulses. Nowadays UV lasers such as nitrogen $(337 \mathrm{~nm})$ or Nd:YAG $(355 \mathrm{~nm})$ are mostly employed, and also IR-MALDI with lasers emitting at $2.94 \mu \mathrm{m}$ are used in analytical practice [71]. The laser shots cause desorption of the matrix-analyte cluster ions as well as matrix and analyte neutrals from the laser interaction region. The analyte ions emerge after ionmolecule reactions with the matrix ions (ideally after gas-phase protonation) or cluster desolvation in the MALDI plume [72]. For its pulsed character, MALDI is preferably coupled to time-of-flight (TOF) mass analyzers.

On one hand, the employment of matrix enabled analysis of high molecular mass species, on the other, matrix presence hampers analysis of the compounds at the low end of the mass range. Because matrix is typically a compound with the molecular mass below 500 Da, (e.g. sinapinic acid, 2,5-dihydroxybenzoic acid, $\alpha$-cyano-4hydroxycinnamic acid (CHCA) etc.) the analysis of low molecular mass species (< $500 \mathrm{Da}$ ) is complicated by the overlapping matrix peaks, that cannot be resolved by TOF-MS. Moreover, matrix molecules tend to form clusters with molecules well up to $1,000 \mathrm{Da}$, thus shifting the overlap problem even further within the mass range. On top of that, the usual MALDI-TOF spectra contain a significant background signal, which is commonly referred to as "chemical noise". This background signal includes both, matrix ions and unresolved ions that span the whole mass range, and further complicate the resulting spectra [69]. Another pitfall of MALDI is the shotto-shot signal variability which is caused by the heterogeneity of sample spots [73]. Nevertheless, MALDI possesses a couple of auspicious advantages: next to the possibility to analyze large biomolecules, MALDI offers a unique opportunity to scrutinize the spatial organization of various sample probes in a planar layout. As a result, MALDI application fields reach far beyond analyses of deposited droplet mixtures.

In 1997, Caprioli et al. [9] demonstrated the use of MALDI mass spectrometry (MS) for the exploration of the molecular composition of tissue sections. The term MALDI mass spectrometry imaging (MSI) has officially been used since that time, although its basic principle was already known and applied for MS analysis of e.g. gels and TLC plates. MALDI-MSI was a logical junction between the MALDI technique that emerged in the late 1980's and secondary ion mass spectrometry (SIMS) imaging that was already well established at the time [74]. During a MALDI-MSI experiment a sample surface is probed with a laser beam in a predefined raster form and a mass spectrum is recorded at each position. As a result, position correlated mass spectra are collected. The final image is defined by the $x, y$ coordinates within a Cartesian grid, and the intensities of a particular $m / z$ visualized in a different colour range [75-77]. An image can be constructed for every single $\mathrm{m} / \mathrm{z}$ present in the spectra. Common samples analyzed with MALDI-MSI are thin 
(10-20 $\mu \mathrm{m})$ tissue sections [78], but in principle any sample thin and flat enough can be explored by MALDI-MSI.

In general, MSI offers several advantages over the other imaging technologies such as positron emission tomography, computer assisted X-ray tomography, nuclear magnetic resonance imaging, quantitative whole body autoradiography (QWBA), or immunohistochemistry (IHC). First of all, MSI does not require any knowledge on the targeted analytes and has no need for any labels. It is a true discovery tool. Furthermore, it allows for highly precise and specific identification of many hundreds of compounds next to each other while preserving their spatial distribution $[77,78]$. Indeed, the spatial information is one of the most significant contributions of MSI. Nevertheless, MALDI-MSI is affected by the general weak points of MALDI described above, thus suffering from several limitations.

The simplest MALDI-MSI sample preparation protocol is based on cutting a sample (e.g. a mouse brain) into thin sections and covering them with a layer of matrix [78]. The overlap problem of matrix and its cluster peaks is manifested for the molecules within the matching mass range. Moreover, as the laser beam rasters over the sample, all molecules present on the sample surface can theoretically be desorbed and ionized. Some molecules tend to ionize more easily, especially when they are present in a higher amount, hence they contribute to ionization suppression effects. These can, together with a limited dynamic range of MALDI-TOF mass spectrometers, lead to incomplete data since the low-abundant molecular species remain "hidden". Lowering sample complexity before MALDI-MSI can significantly help to minimize the ionization suppression and reveal molecules which remain undiscovered.

Separations represent possibly the most effective way of a reduction of sample complexity. The main merit is the reduction of ionization suppression contributing to improved ionization efficiency of low-abundant analytes [79]. Apart from understanding separation as a simple clean-up process, during which undesired compounds are washed off the sample surface, the principle of separation seems to be inappropriate for MSI purposes. It would require sample homogenization, thus resulting in a loss of spatial information: the essential parameter studied by MSI [80]. However, separation methods can provide valuable complementary information through screening the composition of a portion of the to-be-imaged sample and give direction on the further experimental design [81]. From another perspective, the separation resolution of some of the chromatographic and electrophoretic methods such as high performance liquid chromatography (HPLC), thin layer chromatography (TLC) or gel electrophoresis (GE) can further be improved by the employment of MALDI-MSI. The technique can provide deeper insight into the composition of mixtures by imaging the captured eluent or separated analyte bands. Clearly, a combination of MSI and separation methodologies enables an analyst to 
obtain comprehensive information on sample composition. In this review we summarize the possibilities for the reduction of sample complexity that improve MALDI-MSI analysis with a particular attention paid to separation methods. In addition, we review various applications for MSI of separated analytes. The described methodologies contribute to our improved understanding of what can and cannot be visualized by MALDI-MSI.

\subsection{Separations for effective sample clean-up}

One of the advantages of MALDI-TOF is the possibility to analyze very complex mixtures that are not required to be of high purity, since MALDI is relatively tolerant to the presence of salts and buffers [82-85]. However, these contaminants can contribute to altered crystallization and/or ionization suppression, and reduce signalto-noise [86, 87]. For instance, proteins are often treated with surfactants and denaturant agents that are better desorbed from the sample surface during MALDI analysis than the proteins themselves [87].

Various methods of sample purification preceding MALDI target deposition based on off-line separation, such as dialysis, selective extraction (solid phase extraction, liquid-liquid extraction etc.), or chromatographic approaches, have been described so far $[82,88]$. Some of them are further discussed in this review. These conventional methods can, however, be too time-consuming for a quick sample composition screening. Furthermore, they can lead to undesired sample losses.

Membrane based sample supports are used as a fast, effective and straightforward sample purification method, which simultaneously allow for easy sample introduction into a MALDI-TOF mass spectrometer. This approach enables application of a series of washing solutions over the sample spots deposited on top of the membrane. As a result, impurities and contaminants are separated from the molecular species of interest. The principle is also employed for tissue section samples to lower their molecular complexity prior to MALDI-MSI.

\subsubsection{Sample supports for MALDI-MS}

A sample support, typically a polymeric membrane, allows for anchoring samples on the support's surface. The analytes (typically proteins or peptides) bind onto the membrane surface, while the impurities can be washed-off by using a series of rinsing solutions applied on top of the sample spots. Also, further digestion or derivatization of the sample in-situ, i.e. directly on the membrane surface, is enabled [87-89]. In the final stage, a matrix solution is applied on top of the processed spots and the analyte-matrix co-crystals are left to form. In any stage of the procedure the 
membrane is typically placed on a standard MALDI target or a metal disc using a thin layer of adhesive or a double sided adhesive tape [88].

\subsubsection{Polymeric membranes}

An ideal sample support membrane has to (i) allow for binding the analyte upon the membrane surface, (ii) provide sufficient retentive ability during the sample treatment, (iii) enable analytes to resolubilize upon matrix application, (iv) should not add additional complexity to the spectra generated from the membrane, and finally (v) enable the formation of matrix-analyte co-crystals [89]. Various membrane types have been described in the literature so far: nylon [89] and modified nylon (Zetabind ${ }^{\mathrm{TM}}$ ) [89], polyethylene (PE) [86, 87], polypropylene (PP) [87] C8 and C18 discs [87], nitrocellulose [89, 90], regenerated cellulose [91], polyvinylidene fluoride (PVDF) [82, 86, 89], and polyurethane [82, 88]. The membranes offer different capabilities to bind various samples in diverse amounts and also differ in their resistance to chemicals [89].

The separation of the impurities and contaminants is only one of the advantages which the sample support membranes offer. McCombie et al. [82, 88] also demonstrated an accelerated digestion of proteins while using a polyurethane membrane for sample deposition. They explain this phenomenon by protein denaturation upon the membrane adsorption. Worrall and co-workers [87] showed the proteins desorbed from PE and PP membranes require lower laser intensity $(10 \%$ decrease), which results in lower energy ions and better mass resolution. The group also reported on reduced shot-to-shot variability employing these types of membranes. Use of the sample supports for proteinaceous samples is usually well applicable to the analysis of peptides and proteins up to $20 \mathrm{kDa}[82,86,91]$. When higher molecular mass proteins are analyzed the spectra show either comparable results with those obtained from stainless steel probes [82] or present poorer results [91]. An improvement in this field was reported by Blackledge and Alexander [86], who described suitability of PE membranes for the analysis of high molecular mass proteins (over 150,000 Da). The authors explained the improvement with regard to the morphological features of PE membranes. They emphasize their smaller pores, thus large and moreover relatively flat surface. As a consequence proteins are more uniformly deposited onto the membrane surface without deep penetration into its pores. In the end, spectra of higher quality with better peak shape are generated and sensitivity for high molecular mass proteins is significantly improved.

Next to the list of benefits provided by sample supports, a couple of drawbacks have to be pointed out. For instance, several authors reported on difficulties with too little sample adsorbing upon the membrane surface when applying higher volumes of the samples $(1 \mu \mathrm{L})[88,91]$. Washing of the membranes with methanol was 
recommended, as the solvent might possibly lower the intermolecular forces of the polymeric material, thus allowing the analyte a better contact with the broadened membrane pores $[82,88]$. Charging of the membrane surfaces appears in MALDITOF when non-conductive surfaces, such as polymeric membranes, are employed. The charge that builds up during the ionization process gradually alters the surface potential and hence the final kinetic energy the ions obtain. The ions generated from the insulating membrane surface exhibit changing flight times, which result in loss of signal, resolution and/or accuracy [89, 92, 93].

\subsubsection{MALDI sample supports with a modified surface}

Modification of the membrane surface prior to sample deposition can be advantageous in several ways; higher sensitivity, increased spot homogeneity and higher reproducibility. A thin conductive surface modification can be employed for the elimination of sample charging. Such modifications result in improved mass resolution and accuracy as described in the literature [74, 91, 92, 94-96]. For instance, sample spot size was tackled in two independent experiments based on modification of the probes' surface: Millotis and colleagues [94, 95] applied a layer of a matrix-nitrocellulose mixture on top of standard MALDI targets. Schuerenberg et al. [96] described a probe covered with a hydrophobic Teflon layer and deposited hydrophilic gold anchors. In both cases, sample spot diameter was reduced resulting in more concentrated sample spots, hence in increased sensitivity, whereas improved droplets' homogeneity minimized the shot-to-shot variability. Scherl et al. [92] applied a thin layer of gold on top of a membrane in order to ameliorate the charging phenomenon. This resulted in charge dissipation during MALDI-TOF analysis, thus yielding spectra with higher mass resolution.

Zhang and Caprioli [91] used polymeric membranes precoated with matrix: Regenerated cellulose strips were covered with CHCA and further used as a standard sample support. An advantage of matrix precoated membranes is the elimination of sample dilution which leads to decreased sensitivity. The use of matrix precoated MALDI targets for MALDI-MSI has also been described. To point out, MALDIMSI suffers from matrix heterogeneity to a particular extent since a homogeneous matrix layer is essential for an accurate MALDI-MSI experiment. A plethora of methods for matrix application, based on spraying, nebulizing or sublimation have been introduced in the pursuit of the "perfect" matrix layer. Yang and Caprioli recently demonstrated the use of precoated sample targets for MSI of lipids [74]. Matrix was applied on the surface of ITO (indium tin oxide) slides using sublimation. Tissue sections were subsequently placed on top of the modified slides and imaged with MALDI-MS. High robustness and reproducibility was reported by the authors. 


\subsubsection{Washing protocols for MALDI-MSI of tissue samples}

The sample spots on top of a support membrane have several characteristics in common with a tissue section placed on a glass slide. Among them the need for soft washing methods is emphasized. Washing of a tissue section prior to MALDI-MSI is incorporated to separate and selectively remove salts and other undesired molecules from the compounds of interest. The slide with an attached tissue section is either immersed into a beaker with the washing solution, or the solution is applied on top of the tissue using a pipette [78].

Different washing protocols can be applied and are often optimized for the class of analytes studied, so to achieve the most selective separation of the undesired molecules while keeping the molecules of interest in situ. A typical washing protocol for MALDI-MSI includes a series of ethanolic solutions (70-100\%), although organic solvents such as chloroform are employed as well. It is always necessary to keep in mind the solubility of the targeted analytes in the solvents used before the washing steps are performed. Using an improper solvent can either cause a delocalization of the analytes, or these can be washed-off the tissue surface. For instance, when lipids are the subject of tissue a study, lipofilic solvents such as ethanol or chloroform have to be avoided. When analyzing small molecules such as pharmaceuticals, washing is not recommended at all [97]. However, a paper on controlled $\mathrm{pH}$ washing of tissue sections for an increased limit of detection for small compounds has been published recently [98]. Shariatgorji et al. [98] used aqueous buffered solution with $\mathrm{pH}$ adjusted to minimize the solubility of the compounds analyzed. As shown in Figure 2.1, the soluble endogenous compounds causing ionization suppression were removed, while the analytes remained within the tissue sample. The authors report minor to no spatial delocalization of the target compounds. Angel et al. [57] also reported on the benefit of a controlled pH washing protocol for more sensitive detection of lipids from brain tissue. It has to be emphasized that the washing procedure has an ability to change the sample properties vastly and this fact should always be kept in mind [97]. For instance, organic solvents can cause significant damage to delicate and fragile tissue [99]. 

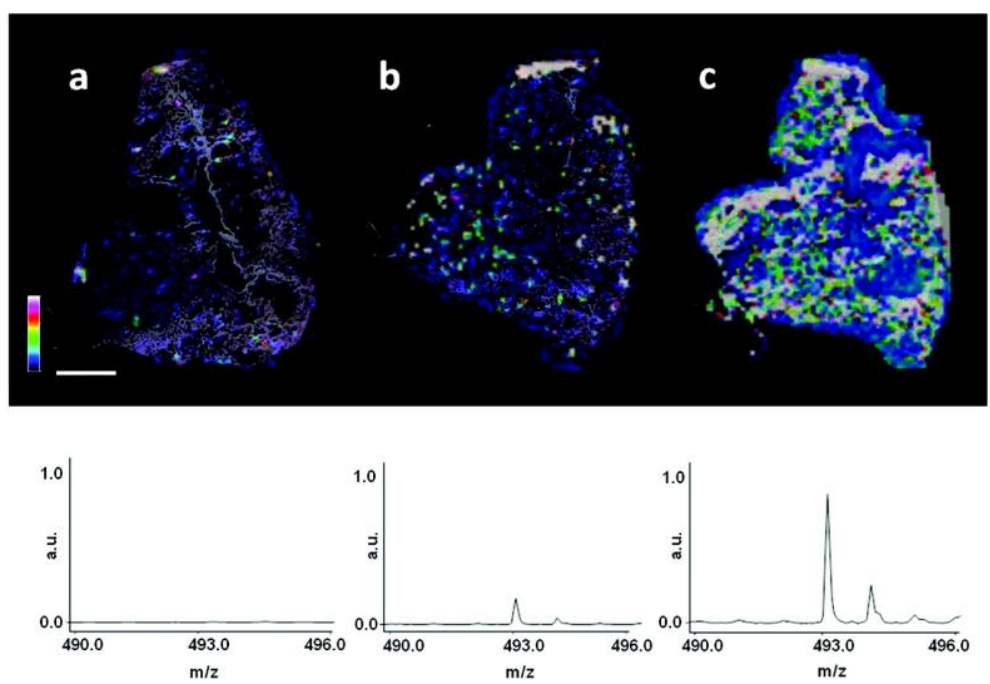

Figure 2.1 Matrix assisted laser desorption ionization-mass spectrometry imaging demonstrates the effect of washing lung tissue sections with a buffered solution. Compound $X$ $(\mathrm{m} / z$ 493.2) was administered to rats by in vivo inhalation. Tissue sections were washed by an aqueous solution with adjusted $\mathrm{pH}$. This ensured insolubility of Compound $X$ which therefore remained in the tissue after application of the washing protocol. On the contrary, the soluble endogenous compounds causing ionization suppression were washed away (a) Non-washed tissue section; tissue section washed (b) in $10 \mathrm{mM}$ ammonium acetate at pH 6 and (c) in 100 $\mathrm{mM}$ ammonium acetate solution at $\mathrm{pH}$ 6. Corresponding summed spectra of the whole tissue sections are shown below the images. (Data displayed in rainbow scale over the same range, scale bar $2 \mathrm{~mm}$.) Reprinted with permission from reference [98].

\subsection{Separations within defined spatial dimensions}

\subsubsection{Planar layout: one- and two-dimensional separations}

MSI is not limited to the analysis of tissue sections [81]; its surface sensitive nature makes the technique suitable for analysis of any probe in a planar layout. MALDI-MSI was for instance used to describe the heterogeneous distribution of analyte molecules within MALDI sample spots [73, 100, 101]. Weidner et al. [100, 101] showed heterogeneous distribution of polymers within droplets deposited on a MALDI target by imaging the spots with MS. Figure 2.2 demonstrates uneven distribution of particular polystyrene homologues within the spots and differences between spectra acquired from the rim and from within the center of the dry droplets. 
(A)

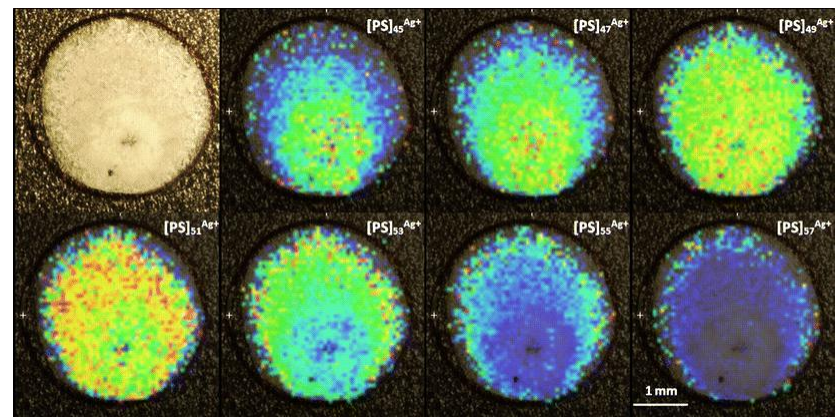

(B)
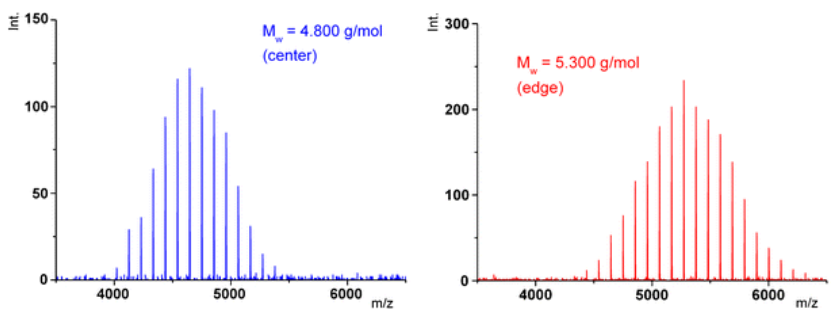

Figure 2.2 Matrix assisted laser desorption ionization-mass spectrometry imaging (MALDIMSI) demonstrates heterogeneous distribution of polystyrene (PS) homologues within sample spots prepared by dried droplet method. $10 \mathrm{mg} / \mathrm{mL}$ of alpha-retinoic acid dissolved in $\mathrm{N}, \mathrm{N}$ dimethylacetamid was used as matrix. PS samples were dissolved in the same solvent at concentration of $1.5 \mathrm{mg} / \mathrm{mL}$. Silver-trifluoroacetate was added to enhance PS ionization. (A) Optical image of the dry sample spot is shown on the top left panel. Ion intensity distribution of every second PS homolog from [PS] $45^{\mathrm{Ag}+}$ to $[P S] 57^{\mathrm{Ag}+}$. Lower mass homologues were found with the highest ion intensities in the middle of the dried spot, whereas higher mass species were located on the droplet rim. (B) MALDI spectra collected on the rim and in the centre of the spot showed $13 \%$ molecular mass deviation. Adapted with permission from reference [100].

Another example of diversity of MSI applications is represented by gels generated with GE and by TLC plates. These techniques are commonly used for the separation of complex mixtures of proteins and various classes of lipids as well as other lower molecular mass compounds, respectively. Deposition of such intricate mixtures on a standard MALDI target would provide an analyst with very complex information and is likely to suffer from ionization suppression effects and low dynamic range. Using membrane sample supports for fast separation of impurities or employing tissue washing protocols does not necessarily lead to a sufficient decrease of sample complexity, and only limited improvement is offered. The impurities are washed-off, but the individual molecular species of lipids and/or proteins remain unseparated. More detailed information on the composition of mixtures can be 
obtained when separation techniques are employed. Figure 2.3 shows a diagram describing briefly how MSI can be involved from two different perspectives:

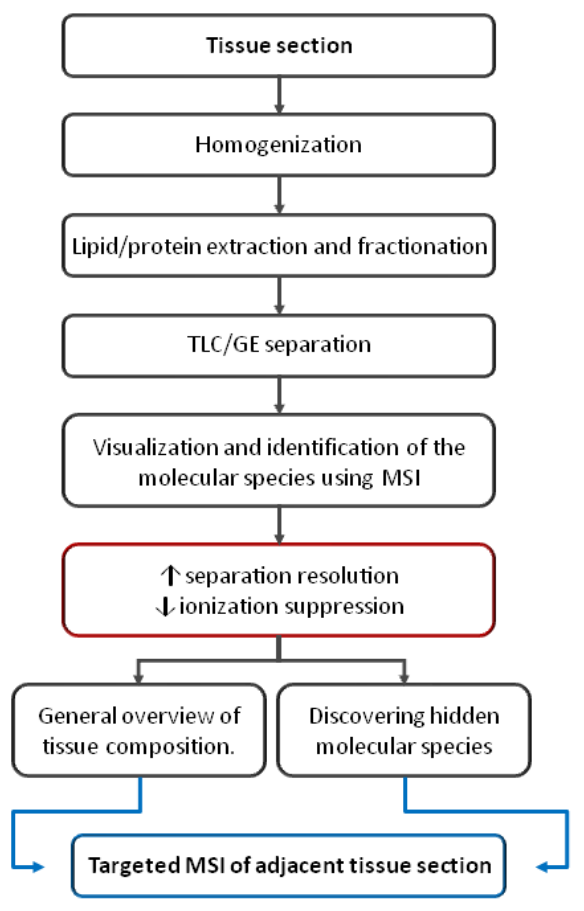

Figure 2.3 Workflow for combining matrix assisted laser desorption ionization-mass spectrometry imaging and separation methods to obtain comprehensive complementary information on a sample composition.

Direct MALDI-MSI of developed gels and TLC plates can be employed. In such case, MALDI-MSI increases the separation resolution of the methods, thus helping to further lower the complexity of the separated mixtures, and provides additional information on samples composition. Moreover, MSI contributes to increased throughput of the analysis. The composition of a tissue sample can be addressed into more detail by creating a homogenate and/or extract from an adjacent tissue section. The obtained lipids and/or proteins can further be separated either by GE or by TLC. Once identified, the separated molecular species can be targeted during MSI of a tissue section. 


\subsubsection{Gel electrophoresis}

Polyacrylamide gel electrophoresis (PAGE) is a one-dimensional (1D) separation technique used for separation of proteins based on their molecular mass. The proteins are first denaturated by sodium dodecyl sulphate (SDS), which results in a charge state proportional to their molecular mass. The proteins are subsequently separated within the gel in the presence of electric field and their molecular mass determined by the migration distance [85]. Isoelectric focusing (IEF) separates proteins according to their isoelectric point, which is determined by their amino acid sequence. IEF employs an in-gel immobilized $\mathrm{pH}$ gradient and electric field. The charge of the protein changes as it migrates through the $\mathrm{pH}$ gradient and the migration stops when the protein reaches the $\mathrm{pH}$ in which it becomes neutral (isoelectric point) [85]. Two dimensional gel electrophoresis (2D-GE) combines PAGE and IEF to separate proteins within one gel, first according to their isolelectric points and subsequently according to their molecular masses [24].

Following separation, proteins are identified using complementary methods. The methods most commonly used include: use of dyes (such as silver stain, Coomasie Brilliant Blue, amidoblack or Poinceau Red) for proteins visualization and subsequent 2D-GE image database searching; immunostaining; sequencing by Edman degradation; and MS [85, 102]. Patterson and Aebersold [93] reported in detail on the mass spectrometric identification of proteins from 2D-GE gels.

MS is a powerful tool for protein identification, and can alleviate most of the limitations of the other methods, such as low sensitivity or potential unavailability of antibodies. For subsequent MALDI-MS analysis, the individual protein bands can either be excised or eluted from the gel and deposited on a MALDI target, or the whole gel can be blotted onto a support membrane, thus obtaining a positive imprint of the separated protein bands. (The latter approach is further discussed in section 2.3.2.2. of this chapter, as a part of the three-dimensional separations.) However, to improve the speed of analysis, elimination of the excision and blotting steps is desired [103], and call for direct MALDI-MS(I) of the gels is emphasized [104]. For instance, Ogorzalek-Loo et al. [104] described MALDI-MS approach for characterization of proteins directly from polyacrylamide gels. They claim that their approach is suitable for both $1 \mathrm{D}$ and $2 \mathrm{D}$ gel separations. A requirement of significant importance, which cannot be taken for granted, is that the gel thickness does not exceed $10 \mu \mathrm{m}$. The authors point out and discuss in detail the fragility of thin gels. As reported, increase of mechanical stability can be achieved by soaking the still wet unstained gels into sinapinic acid solution. Sinapinic acid is believed to act as a plasticizer.

Even though some authors proclaim 2D-GE being the method with the highest resolution for protein separation [85], the actual separation power is often 
insufficient, thus resulting in single spots containing more than one protein species [105]; more than $10 \%$ of the protein bands actually contain more than one protein [103]. The group of Yang [105] resolved this problem by subjecting the spots excised from 2D-GE gel to complementary liquid chromatography tandem mass spectrometry (LC-MS/MS) analysis. Contribution of MSI to direct analysis of the 2D-GE gels would help to improve separation resolution by revealing spots of comigrating proteins or identical proteins which migrate to different positions within the gel [105]. Gels after protein separation by IEF were rastered with a laser beam (step size of $0.3 \mathrm{~mm}$ corresponded to roughly 500 mass spectra from a single band [103]). Subsequently a dedicated software was employed to obtain so called "virtual 2D gels" $[103,106]$. The generated 2D images were based on isoelectric point and molecular mass determined by MALDI-MS instead of SDS PAGE [104, 106]. Walker and co-workers [103] compared this approach with the conventional 2D-GE and showed that the virtual 2D gels enable identification of proteins, which remain unresolved under 2D-GE conditions. They further report the accuracy of molecular mass determination is 100-500 times better for the virtual 2D gels [85, 103]. An example of an acquired virtual 2D gel is shown in Figure 2.4.

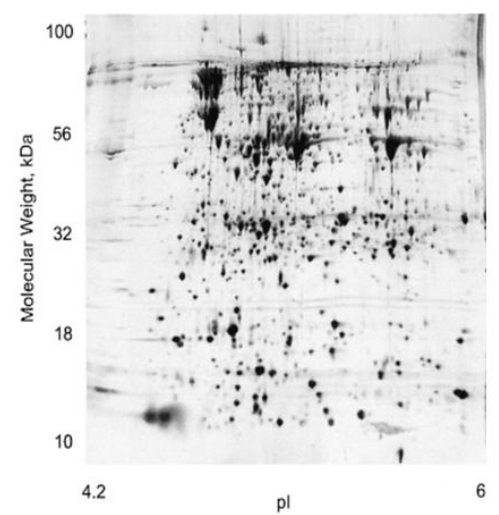

(A)

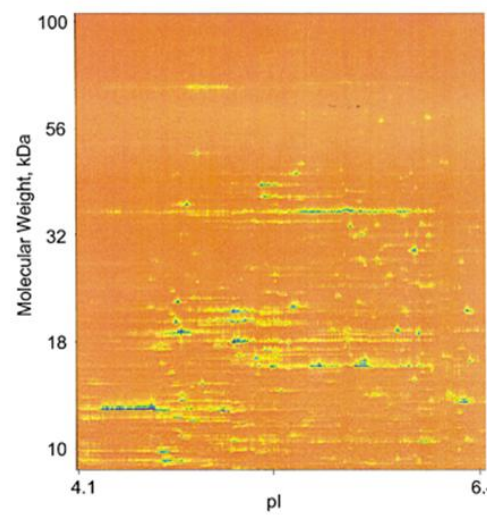

(B)

Figure 2.4 Comparison of two visualization techniques for separated E. coli whole cell extracts. A) Gel after a conventional two-dimensional gel electrophoresis (2D-GE), proteins visualized with silver-staining, B) virtual $2 D$ gel acquired by MS scanning over in-gel protein bands separated by isoelectric focusing. Specialized software tool was used to reconstruct the $2 D$ image. Adapted with permission from reference [103].

It has to be pointed out that successful MALDI-MSI analysis of gels requires a lot of practical considerations. Particular attention has to be paid for instance to vacuum compatibility and stability of the gel, to washing-off the crystallization 
disrupting contaminants while preserving the distribution of the proteins within the gel, or to a proper matrix-analyte co-crystals formation [103]. Charging phenomena have been observed, since gels act as insulators [107]. These factors also limit the successful application of MALDI-MSI for the analysis of 2D-GE gels.

\subsubsection{Thin layer chromatography}

TLC is based on distribution of an analyte between two phases: a stationary phase, anchored on a solid support, and a mobile phase transported by capillary forces through the layer of the stationary phase [108]. 2D separations represent an attractive application of TLC. In such case, two systems of mobile phase are used in a subsequent order to develop a single TLC plate [109], thus increasing the peak capacity and separation resolution.

The scope of the most routine TLC applications includes fast monitoring of drugs of abuse and toxic substances in biological fluids, water purity surveys, quality control in pharmaceutical industry [108], and a rapid screening of impurities of the in-house synthesized compounds. It is also widely used in lipid research for separation of individual lipid classes, see also Figure 2.5 [110]. The scope of the analytes typically targeted by TLC is represented by molecular species with mass roughly below 1,000 Da [107]. The traditional detection methods for TLC are based on UV and fluorescence detection or on the use of colour reagents and derivatizing reactions. Another group of detection systems for TLC is based on optics, e.g. scanning densitometry or video densitometry, the latter one replaced the antiquated technique of photographing the TLC plates [108]. MS(I) falls to the third category of TLC detection systems. 


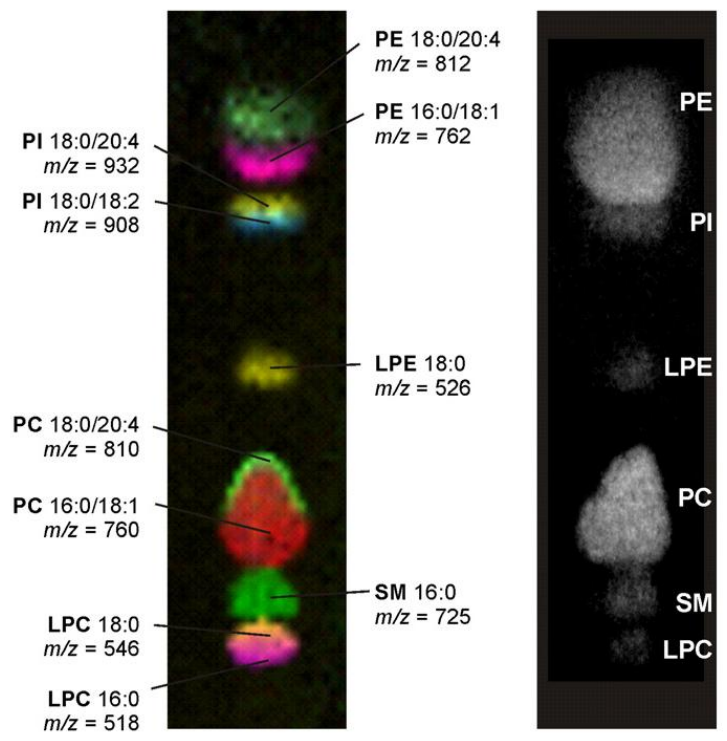

Figure 2.5 Lipids from hens' egg yolk separated by thin layer chromatography (TLC) and visualized by matrix assisted laser desorption ionization-mass spectrometry imaging (MALDIMSI) (left panel) and by densitometry (right panel). MSI clearly separated lipid species within individual lipid classes (PE, phosphoethanolamine; PI, phosphoinositol; LPE, lysophosphoethanolamine; PC, phosphocholine; SM, sphingomyelin; LPC, lysophosphocholine), whereas the conventional approach could tell apart only the particular lipid classes. MS imaged two different spots for most of the lipid classes and differentiated lipids with longer and shorter fatty acyl residues. MALDI-MSI increased separation resolution of TLC and provided deeper understanding of the mixture composition. Reprinted with permission from reference [110].

Since development of electrospray ionization (ESI), coupling HPLC to MS has become well-established and routinely used. Also TLC can be coupled to ESI-MS: The off-line TLC-ESI mode is based on solvent elution or extraction from the TLC separated bands and a subsequent injection of the independent fractions into a mass spectrometer [111]. Obviously, such an approach is very laborious and time consuming [108, 111]. Moreover, the extraction/evaporation procedure can lead to significant sample losses and a sensitivity decrease. On the contrary, the on-line TLC-ESI interface ensures faster, more robust and sensitive detection. However, the price for reduced risk of sample losses is the requirement for highly sophisticated instrumentation [109]. For these reasons, the most efficient way for direct TLC-MS coupling is based on desorption ionization techniques such as MALDI [109,111] (or DESI). With MALDI the analytes can directly be desorbed from the plate surface omitting the imprecise and lengthy evaporation or elution process. 
In general, the compatibility of TLC plates for direct MALDI-MS analysis is higher than that of gels. The pitfalls of the interface are mainly represented by charging phenomena [107], and possible contamination of the mass spectrometer by silica residues [112]. Unlike in GE, use of matrix represents a major limitation in MALDI-TLC with regard to the molecular masses of the analytes typically studied. Application of the solvent based matrices also causes band spreading. Moreover, attention has to be paid to a proper formation of analyte-matrix co-crystals, which can be hindered by the presence of silica gel [113]. Therefore, other desorption ionization techniques, such as DESI (desorption electrospray ionization) [114, 115], or SALDI (surface-assisted laser desorption ionization) [116], have also been employed in MS(I) analysis of TLC plates. However, TLC-MALDI-MS(I) has successfully been used for analysis of pharmaceuticals [117], drugs of abuse [118], lipids [113, 119], glycolipids [120, 121] oligosaccharides [122], and also small peptides [123]. Ivleva et al. [120, 121] investigated direct MALDI analysis of TLC plates with an FTMS instrument equipped with a vibrationally cooled MALDI ion source. The vibrational cooling during ion formation in the MALDI plume prevented fragmentation of labile gangliosides species. As a result, straightforward identification of individual gangliosides was achieved even within incompletely spatially resolved TLC bands.

Furthermore, the potential of IR-MALDI for TLC read-out was addressed by several research groups [113, 117]. As reported by Rohlfing et al. [113], IR-lasers are capable of penetrating much deeper into a TLC plate in comparison to UV-lasers. As a consequence, analytes from the deeper silica gel layers are efficiently released. Since IR-MALDI enables the usage of a wider range of matrices, e.g. glycerol, problems with decent analyte-matrix co-crystals formation are ameliorated [113].

The single bands on a TLC plate often consist of more than one molecular species. By MSI of the plate, the separation resolution is increased over the conventional read-out and the individual analytes within one band are differentiated. This is shown in Figure 2.5 where lipid species were separated on a TLC plate, which was subsequently imaged using MALDI-MS. Kuwayama et al. [118] demonstrated the use of TLC-MALDI-MSI for analysis of drugs of abuse in biological samples. They alleviated the need for lengthy sample preparation by TLC separation of 11 psychotropic compounds. They detected the drugs of abuse and visualized the spots on a TLC plate with MALDI-MSI. MALDI-MSI is demonstrated to be capable of providing a far more robust molecular identification when compared to the information based on retention factor only. The application of tandem MS in these MALDI-MSI experiments increases the identification reliability. 


\subsubsection{Blotting: adding the z-dimension}

Generally, during a blotting experiment the analytes, previously separated in one or two dimensions, are further detached from the separating environment in the vertical direction. Nevertheless, separation is not always necessary since a solid substrate such as tissue section can be blotted directly. Blotting augments sensitivity of MS detection and further lowers sample complexity. It also facilitates sample transfer to a MALDI mass spectrometer by capturing the analytes of interest onto a membrane surface, thus encompassing the fragile gels and non-inert silica plates. The overall advantages of the membranes used in MALDI-MS analysis have already been summarized in section 2.2.1. of this chapter.

\subsubsection{Blotting TLC plates}

Blotting helps to prevent the band broadening during direct matrix application on a TLC plate [111]. Gusev and co-workers [124, 125] introduced an alternative methodology based on crystallization of matrix on an inert substrate and subsequent imprint of the matrix layer on top of the TLC plate. In other work, blotting of TLC plates improved IHC based detection of glycosphingolipids (GSL) and phospholipids separated in a TLC experiment [126]. The first blotting experiments involved transfer of GSL onto a nitrocellulose membrane [127]. Since the reproducibility and efficiency of that experiment were low, Taki and co-workers [128] tested the suitability of other polymeric membranes, and finally substituted the nitrocellulosebased probe with a PVDF membrane. In later work, they replaced the IHC detection with MS [129]: the bands of blotted GSL were cut out of the PVDF membrane and analyzed with SIMS. Kasama et al. [130] demonstrated the TLC-blot-SIMS method for screening GSL composition of rat mammary tumour cell lines with different metastatic potentials. Guittard et al. [131] adopted the blotting protocol of Taki's group [128] and compared direct MALDI analysis of TLC plates and TLC blots. The group reported on better spectral quality of the membrane-fixed analytes, demonstrating higher sensitivity, higher signal-to-noise ratio and better mass resolution.

Gusev et al. [124] reported on imaging of TLC using MALDI-MS. Their work was based on microprobe mode MSI obtaining contour-plots. The pioneering work on MALDI-MSI of TLC blots was done by Taki and co-workers [126, 132] .Their blotting protocol is performed as follows: a developed. TLC plate is dipped into a blotting solution for several tens of seconds and placed on a glass plate. Subsequently, a PVDF membrane, a Teflon membrane and a glass fibre sheet paper are placed on top of the TLC plate, followed by pressure from an iron heated to $180^{\circ} \mathrm{C}$ for $10-30 \mathrm{~s}$. The benefits of Taki's TLC-MALDI-Blot-MSI method were 
demonstrated in several applications [112, 133, 134]. Similarly to direct MSI of TLC plates, imaging of the blot membranes increases the separation resolution. For instance, the combination of TLC and MSI of the blot membranes helped to distinguish two molecular species of GSL GM1, which created only one band on the TLC plate when detected by primuline based staining [112]. In another publication the glycolipidome of patients with Alzheimer's and Parkinson's disease was compared to control samples [134]. Significant differences in lipid composition within the hippocampal region were reported. This application demonstrated the complementarity between MSI and separation techniques: the brain tissue extracts were separated using TLC and the lipid species were further separated from the plates in the third dimension by employment of a blotting step. High separation resolution was achieved and brain lipid composition was studied by MSI of the blots obtained. (See Figure 2.3.)

\subsubsection{Blotting gels}

Most of the methods for protein identification from gels take the advantage of transfer of the separated proteins onto a more robust polymeric membrane [135]. The protein spots are cut out of the membrane and homogenized for identification by Edman degradation, hence the information on protein position within the gel/membrane is lost. On the contrary, during IHC based detection the imprint of proteins on the membrane remains intact, hence the proteins are identified while their spatial distribution is preserved. The immunoassays, however, suffer from significant drawbacks: dependency on existence of specific antibodies and limited possibility of simultaneous detection of multiple analytes. Those pitfalls are successfully ameliorated by MALDI-MSI. In general, MALDI-MS(I) analysis of proteins blotted onto a membrane surface is more feasible when compared to gels owing to the higher stability of membranes. Moreover, the proteins are transferred in a highly purified state free of contaminants such as SDS, salts or buffers [83, 136, 137], hence the ionization suppression effects are reduced.

MALDI-MSI of 2D-GE separated and subsequently blotted proteins has not been established for routine analysis yet (except of the molecular scanner tool discussed in section 2.4.2. of this chapter). Vaezzadah and co-workers [81] reported on MSI of tryptic peptides blotted onto a polymeric membrane after IEF. Their approach allows a MSI based preview of a proteome to be obtained, and provides information such as relative concentration of proteins, presence of contaminants, and overall complexity of the sample. Based on this knowledge, strategies for further proteome analysis can be designed [81].

A number of MALDI-MS applications for analysis of blot-transferred proteins have been described and reviewed extensively [83, 135-139]. Some authors paid 
attention to the comparison of UV- and IR-MALDI for on-membrane captured proteins analysis and the suitability of membrane types [83, 138, 139]. It was found out, that while proteins on sample support membranes are well desorbed with UVMALDI, the spectral quality of blotted proteins is significantly lower [139], and IRMALDI becomes more suitable [83, 137, 138]. Further, several matrix application methods to avoid the protein bands spreading were tested. Strupat et al. [83], for instance, recommended matrix application by soaking the wet membrane into an aqueous matrix solution rather than wait until the membrane becomes dry and then rewetting with organic solvents during matrix application. The organic solvents relocate the proteins within the membrane and an accurate spatial information is lost [137]. Another possible solution of band spreading prevention might be blotting the gel on a membrane already precoated with matrix as previously performed for tissue sections [140]. Application of this approach to gel-separated proteins however, is yet to be demonstrated.

\subsubsection{Blotting tissue sections}

The concept of blotting tissue surfaces to obtain a mirror molecular image was introduced in the very first paper on MALDI-MSI [9]. Caprioli and co-workers [9] acquired tissue imprints by contacting the exposed cells of a freshly cut tissue with various targets: cellulose membrane, surface covered with $\mathrm{C} 18$ beads and stainless steel probe. Blotting of the tissue sections led to selective separation/extraction of proteins and peptides from interfering compounds such as salts and lipids. Chaurand et al. [84] demonstrated successful MALDI-MS profiling of tissue transfer blots on a conductive carbon filled PE membrane. The chemical properties of the membrane allowed for a protein separation/extraction from the tissue surface and for a subsequent water based washing. Vidová et al. [141] demonstrated transfer of lipids by blotting tissue surface onto a nano-assisted laser desorption ionization (NALDI) target followed by matrix-free NALDI-MSI. Bunch et al. [140] demonstrated the applicability of the blotting approach for low-molecular compounds. They used a matrix (CHCA) precoated cellulose membrane to transfer ketoconazol (observed at $m / z$ 531.19) by blotting a medicinal shampoo treated porcine skin sample. The group investigated also a quantitative approach of their blotting methodology, obtaining a good linearity when a matrix peak was employed to normalize the signal.

Acquisition of a positive imprint of a tissue section represents a separation step where only the z-dimension is employed. As demonstrated, blotting leads to effective separation of compounds of interest, thus decreasing the tissue complexity and reducing ionization suppression. The imprinted areas can be washed by applying rinsing solutions. If performed carefully, spatial information is preserved [9, 140]. 
Special attention also has to be paid to timing of the blotting to avoid tissue dehydration [84].

Another application of tissue blotting was published by van Hove et al. [142]. The group presented an alternative method for tissue washing based on close contact of a tissue section with a wetted fiber-free paper. Separation of salts from the sample surface was demonstrated. The advantage is that this approach allows for washing of delicate tissues or samples, such as those mounted with double-sided tape, that cannot be washed by conventional methods. The method also allows for washing a selected area of a tissue sample exclusively.

\subsection{One step blotting \& digestion}

\subsubsection{Protein identification strategies for MS}

Determination of molecular mass of an intact protein is not sufficient for its unambiguous identification [138]. MS strategies for protein identification have been reviewed in the past $[85,93,143,144]$ and a concise review on protein identification using MSI was recently published by Mascini and Heeren [145]. Two strategies are distinguished based on the absence or presence of a protein pre-digestion step within the MSI workflow, i.e. top-down and bottom-up approaches [145, 146]. In a topdown experiment, intact proteins are directly analyzed in a mass spectrometer. By subsequent MS/MS analysis, the proteins are fragmented according to well-described patterns, which allow for protein identification using specific databases [78, 145]. The bottom-up approach requires a protein digestion preceding MSI. The emerging peptides are analyzed by MS and the subsequent identification process is either based on peptide-mass-fingerprinting (PMF) or on MS/MS analysis [145]. The topdown MS strategy is limited by the performance of the currently available instrumentation. For instance, when using a MALDI-TOF mass spectrometer it is usually impossible to analyze high-molecular mass proteins $\left(\mathrm{M}_{\mathrm{m}}>30 \mathrm{kDa}\right)[78,145$, 147]. The mass dependent sensitivity drop-off causes a loss of signal for the larger proteins [78]. Protein digestion brings the proteins into a detectable mass range with sufficient sensitivity [78].

The protein digestion for a bottom-up approach can be performed before the gel separation [81], directly in gel after 2D-GE [148], on a tissue section before a MSI experiment [149], as well as on a support membrane after gel/tissue blotting [84, 138] or sample deposition [89]. The in situ digestion approach limits additional sample handling and therefore appears to be the most convenient. However, presence of significant interfering background signal is stressed especially for tissue samples [145]. A strategy for lowering complexity of digested samples while preserving the 
protein spatial distribution within the gel/membrane/tissue has been presented in the literature. The approach is known as the molecular scanner and is well suited for MALDI-MSI analysis.

\subsubsection{Molecular scanner}

The principle of molecular scanner was introduced by Hochstrasser's group in Geneva in 1991 [150] as a tool for automated high-throughput proteome research, and since then a number of papers have been published on the topic [150-155]. The concept is based on three adjacent layers: a gel with separated proteins or a tissue section, a hydrophilic membrane with covalently bound trypsin, and a polymeric (PVDF) capture membrane [146]. By applying an electric field, the proteins migrate through the enzymatic membrane and resulting proteolytic peptides are captured on a collection membrane. In a subsequent step this capture membrane is imaged using MS and the final intensity based plot is reconstructed. Several software packages were developed for the evaluation of the 2D proteome images. Müller et al. [154] reported in detail on visualization and analysis of the molecular scanner generated peptide mass spectra. Data generation and analysis was thoroughly described [153, $155]$.

The above-described protocol is known as "one step digestion transfer" (OSDT). Another assessment includes digest of the proteins directly in the gel. This "parallel in-gel digestion" approach leads to more efficient digestion and is particularly suitable for proteins which are too large to be transferred through the enzymatic membrane. It suffers, however, from loss of mass resolution for low molecular mass peptides [151]. The best results were obtained when the proteins were first in-gel pre-digested with a small amount of trypsin and followed by standard OSDT (“double-parallel digestion" approach) [151].

One of the general limitations of the molecular scanner is that some proteins are more easily extracted from the gel/tissue surface than the others, which results in only a partial protein content analysis [78, 147]. The spatial resolution of a scanning experiment is also a limiting factor. As the whole protein band cannot be concentrated into a single spot on the collecting membrane, the sensitivity of the method is limited [152]. This drawback can be overcome by the MSI approach, especially by the employment of MSI in microscopic mode [146]. Luxembourg and co-workers [146] separated proteins in one dimension and performed the molecular scanner experiment. The collecting PVDF membrane was subsequently covered with matrix and a gold layer to avoid charging effects. The prepared membrane was imaged using MALDI-MSI in microscopic mode and allowed for high spatial resolution imaging of the captured peptides. Rohner et al. [147] reported on application of the molecular scanner approach for analysis of tissue sections by the 
means of MSI. The capture membrane was MS imaged to visualize proteolytic peptides generated from the tissue surface. The preliminary results showed preserved spatial information and reduced ionization suppression [147]. The working protocol, however, needs additional optimization.

\section{Box 1. Microprobe mode vs. microscope mode MSI}

Microprobe mode MSI is more frequently employed approach of MSI [78]. The focused laser beam rasters the sample surface and a mass spectrum is collected at each raster point. The spatial resolution is determined by the laser spot diameter $[78,156]$. The final image is defined by the positions of mass spectra collected and intensity of a particular $\mathrm{m} / \mathrm{z}$ throughout these spectra. The images can be represented in a form of colour scaled density maps, 3D intensity profiles [124, 153] or contourplots $[124,137]$. In microscope mode an unfocused laser illuminates a larger area of the sample surface. The ion optics preserves the ion spatial distribution of the desorbed ions until they reach a position sensitive detector [157]. Microscope mode MSI generates highly resolved ion images with spatial resolution up to $4 \mu \mathrm{m}$ within the laser spot that has a diameter of $\sim 200$ micrometers $[78,156]$. Large data sets are generated in microscope mode MSI due to the high spatial resolution involved. It is advantageous to combine the microprobe mode for screening with subsequent microscope mode high spatial resolution of targeted compounds [146].

\subsection{Column and capillary separations}

\subsubsection{Direct MALDI interface}

Capillary electrophoretic (CE) and liquid chromatographic (LC) separation methods are nowadays commonly coupled to mass spectrometers, which allow for highly sensitive and selective identification of the analytes. The interface of choice for coupling LC and CE to MS is ESI since it allows for direct introduction of liquid samples into a mass spectrometer, i.e. it allows for a convenient on-line coupling. However, in comparison to ESI, MALDI has several important benefits, some of which have been already addressed in the previous text, i.e. it is less sensitive to the presence of contaminants, it requires significantly smaller amounts of sample, offers faster analysis and thus higher throughput, and provides simpler mass spectra since singly charged molecular ions are created [79, 94, 107]. The possibility of MS/MS analysis is a prerequisite for an accurate analyte identification. In an on-line LC-ESI approach MS/MS of a selected $\mathrm{m} / \mathrm{z}$ must be performed within the elution time of the analyte. MALDI can overcome this problem since it enables re-analyses of a capture 
spot until the sample is completely used [79]. The advantage of CE- or LC-MALDI becomes obvious when analyzing very complex mixtures of protein and peptide mixtures that are often too complex for an unambiguous identification [94].

Several interfaces of coupling column and capillary separation to MALDI-MS have been described in the literature and some of them have been reviewed by Gusev [107]. The simplest approach is an off-line fraction collection of the eluent with a subsequent spotting of sample array on a MALDI target and addition of matrix. However, on-line coupling significantly improves the interface [94, 158]. It can be achieved either by deposition of discrete fraction droplets [94, 158], or by deposition of a continuous flow of the eluent. The eluent flow is either coupled to a flow of matrix, so that both solutions are deposited on a MALDI target simultaneously $[159,160]$, or utilizes MALDI targets that are precoated with matrix [79, 94, 95].

Independently of the CE/LC-MALDI interface, the MALDI experiment itself is typically achieved by analysis of discrete, individual spots. However, this approach is ineffective and time-consuming, and detailed information on the retention times of particular analytes is lost. On the contrary, the nature of MSI makes it compatible with coupling to micro-scale separation methods while spatially preserving the retention time information, and significantly easing the data collection [160]. An image of an ion with a particular $\mathrm{m} / \mathrm{z}$ can be constructed from the deposited eluent with MSI as a detection method. The position of this ion now represents its retention time within the separation process [160].

For instance Zhang et al. [160] reported on the advantageous LC-MALDI-MSI combination. They described separation of neuropeptide mixtures, continuous flow deposition of the eluent on a moving MALDI target with subsequent MSI analysis of the deposited eluent lines. Their practical workflow of the image-based analysis of a mass spectrum with complex and low abundant peaks is shown in Figure 2.6. Their approach enabled identification of a significantly higher amount of peptides when compared to traditional fraction collection followed by a spot-by-spot MALDI analysis ( $81 \%$ vs. $66 \%$ of total protein sequence for MSI and spot-by-spot analysis, respectively). In another publication, Zhang et al. [80] utilized MALDI-MSI in a robust off-line platform coupled with pressure enhanced capillary electrophoresis. 2D separation combining LC and CE with subsequent MALDI-MSI analysis was evaluated for studies of neuropeptide mixtures [161]. It was the first, and to-date only, combination of 2D column/capillary separation with MSI [161]. 


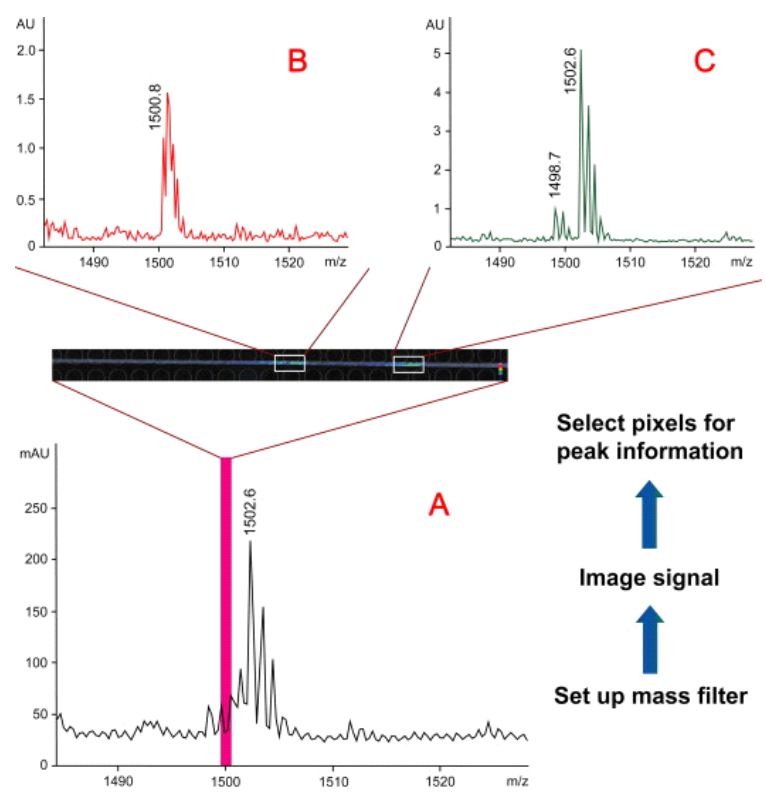

Figure 2.6 Workflow for complementary use of liquid chromatography $(L C)$ separation and mass spectrometry imaging (MSI). A) MS full scan of a bovine serum albumin tryptic peptide $(\mathrm{m} / \mathrm{z}$ 1502.6) showing the mass range from $\mathrm{m} / \mathrm{z} 1490$ to $\mathrm{m} / \mathrm{z}, 1520$. Low abundant $\mathrm{m} / \mathrm{z}$ are suppressed by the main signal at 1502.6; 1 Da wide selected mass filter is represented by the pink strip. The corresponding image for the selected mass filter signal is enlarged, and highlighted in the white boxes, there are two individual peptides with different retention times. $\boldsymbol{B})$ and $\boldsymbol{C})$ Mass spectrum of a particular region of interest corresponding to the separated peptides. The peaks at $\mathrm{m} / \mathrm{z}, 1500.8$ and 1498.7 are now observed with better resolution. The three-step approach 1) select mass filter, 2) obtain image, 3) extract ions from pixel/region of interest, can also be used when looking for unknown analytes within complex mixtures by screening the mass filter across the entire mass spectrum. Reprinted with permission from reference [160].

\subsubsection{Validation and corroboration of MSI results}

Ionization suppression represents a particular problem in quantitative MSI (qMSI), which aspires to become a fully established quantitation method for e.g. pharmacokinetics and drug stability studies. Importantly, the degree of ionization suppression for a single analyte is tissue type dependant. This fact complicates qMSI performed in whole body tissue sections, where multiple tissue types are present. The imperfections in the matrix layer homogeneity as well as MALDI shot-to-shot variability account for further bottlenecks. Several approaches of alleviating the MALDI-qMSI pitfalls have been recommended and/or tested, and publications reporting on successful qMSI can be found in the literature [162-165] and some of the works referred to therein. Pirman et al. reported on the use of isotopically 
labelled internal standards for MSI [166]. However, to validate results obtained by qMSI, well-established quantitative techniques such as LC-MS/MS [162-164] or QWBA [162] are still needed. These techniques lack some of the advantages of qMSI, such as label-free spatial information but provide reliable quantitative data. Beside the corroboration in quantitative research, LC-MS/MS is also used to validate qualitative information obtained by MSI. This approach became useful for instance during search for new natural products [167] and biomarkers [168], or for confirmation of peptide identification through accurate mass analysis [169].

\subsection{Conclusions and future perspectives}

In this review, we have discussed the benefits of strategies that combine analytical MALDI-MSI and various separation techniques. Both, MALDI-MSI and separation techniques provide an analytical scientist with information on the molecular composition of a sample. The essential contribution of MSI is the spatially correlated molecular information. The separation techniques have the ability to significantly reduce ionization suppression, hence improve ionization of lowabundant molecular species. A comprehensive report on sample composition is obtained by their combination in a single analytical approach. However, there is still room for improvements.

For instance, the blotting technique, introduced at the dawn of MALDI-MSI, has remained relatively unexplored, especially with respect to the tissue sample analysis. As demonstrated in several applications the blotting process preserves the spatial distribution of the studied molecules when performed carefully. It is known that different classes of compounds vary in their ability to be adsorbed onto diverse polymeric materials. Moreover, the z-dimension based separations could be performed selectively by further derivatization and/or functionalization of the polymeric probes. As a result only particular analytes would be separated from a pool of the compounds present on a tissue surface.

Another set of improvements can be introduced with the new ionization techniques. Dramatically fast developing ambient ionization techniques, such as laser ablation electrospray ionization, offer the advantage of analysis of vacuum incompatible samples, and of little to no need for sample preparation steps. The problem of bands broadening upon matrix application would therefore be overcome. Furthermore, ambient ionization techniques would allow for direct analysis of gels with separated proteins without significant problems with gel in-vacuum stability

Discovery or development of a universal analytical method capable of unbiased and highly sensitive detection of all molecular classes within one experiment is still far from achieved. Until its introduction, the most relevant information on a sample 
composition will be obtained based on combinations of existing complementary analytical methods, which are being improved constantly. 


\section{Ambient Mass Spectrometry Imaging and Ion Mobility Separation}

Based on: Karolina Škrášková, Emmanuelle Claude, Emrys A. Jones, Mark Towers, Shane R. Ellis, and Ron M. A. Heeren: Enhanced capabilities for imaging gangliosides in murine brain with desorption electrospray ionization mass spectrometry coupled to ion mobility separation. (2015) Manuscript submitted to Methods. 


\section{Desorption electrospray ionization and MALDI mass spectrometry imaging coupled to ion mobility separation: Enhanced lipid information}

The increased interest in lipidomics calls for improved yet simplified methods of lipid analysis. Over the past two decades, mass spectrometry imaging (MSI) has been established as a powerful technique for the analysis of molecular distribution of a variety of compounds across tissue surfaces. Matrix-assisted laser desorption ionization (MALDI) MSI is widely used to study the spatial distribution of common lipids. However, a thorough sample preparation and necessity of vacuum for the ionization process, might hamper its use for a high through-put lipid analysis. Desorption electrospray ionization (DESI) is a relatively young MS technique. In DESI, ionization of molecules occurs under ambient conditions, which alleviates sample preparation. Moreover, DESI does not require application of external matrix, making detection of low mass species easier due to the lack of chemical matrix background. However, irrespective of the ionization method, the final information obtained during a MSI experiment, is very complex and its analysis becomes challenging. It was shown that coupling MSI to ion mobility separation (IMS) simplifies imaging data interpretation. Here we employed DESI and MALDI MSI for a lipidomic analysis of the murine brain using the same IMS enabled instrument. We report for the first time on the DESI-IMS-MSI of multiply-sialylated ganglioside species, as well as their acetylated versions, which we detected directly from the murine brain tissue. We show that poly-sialylated gangliosides can be imaged as multiply charged ions using DESI, while they are clearly separated from the rest of the lipid classes based on their charge state using ion mobility. This represents a major improvement in MSI of intact fragile lipid species. We additionally show that complementary lipid information is reached under particular conditions when DESI is compared to MALDI imaging.

\subsection{Introduction}

The brain is an extremely complex organ. It is a lipid dense structure that consists of $60 \%$ lipids [32]. Lipidomics is a relatively new research area which aims to assess all lipids within a cell and explain their function and inter-connectivity [31, 170]. The analysis of lipids becomes very challenging due to their high molecular complexity and a wide array of lipid species present in biological samples [30, 31]. Mass 
spectrometry (MS) is widely used for lipidomic studies. Its strength stems from the simultaneous and differential detection of individual lipids [31, 171]. A standard lipidomic study involves extraction of the lipids from a tissue and a subsequent MS analysis of the extracts (with or without prior chromatographic separation). Any spatial information is lost during the extraction process.

Mass spectrometry imaging (MSI) [62] is a surface analysis technique which enabled analysis of lipids in situ, e.g. directly within thin brain sections. Hence, their anatomic localization within the brain can be mapped. A sample surface is probed with a stream of particles (e.g. photons, primary ions, or charged droplets) during a MSI experiment [172]. Upon the sample bombardment, molecules are desorbed from the sample surface, ionized and their molecular mass is subsequently determined in a mass analyzer. Since a mass spectrum per sampling position is recorded, an overview of a molecular composition per sampling location is created. A reconstructed MS image reflects the spatial distribution of a single molecular species (ion) across the probed sample surface.

Matrix-assisted laser desorption ionization (MALDI) is the most routinely employed ionization technique for MSI. MALDI has been successfully applied in imaging of lipids across different tissue types such as rodent brain [40, 173], retinal tissue [174] or tumor sections [41]. MALDI makes use of a thin layer of matrix (typically a low molecular aromatic organic acid) deposited on a sample surface. Upon irradiation the matrix induces analyte ionisation greatly minimises fragmentation of the analytes of interest. However, the use of a matrix can also increase spectral complexity. Analysis of low molecular mass compounds is hampered by the presence of matrix due to additional, overlapping peaks. Some matrices such as $\alpha$-cyano-4-hydroxycinnamic acid (CHCA) tend to form clusters, hence the overlap region is extended up to 1,000 Da within the mass range [175].

MALDI is well suited for imaging of phospholipids including glycerophospholipids (GPL) and ceramide-based sphingomyelines (SM). Sulphatides (ST), another type of ceramide backbone containing lipids, are also easily ionized in MALDI. Certain types of phospholipids, namely phosphocholines (PC) and SM, contain quaternary nitrogen, which makes them "pre-ionized". As a result, they dominate mass spectra in positive mode, and can often suppress the detection of other lipid classes [176].

On the contrary, the ionization of complex glycolipids such as gangliosides is challenging with MALDI. Gangliosides contain a mono- to poly-sialylated oligosaccharide chain of variable length attached to the ceramide portion of the molecule. Gangliosides are particularly abundant in nervous system, i.e. in the brain. Changes in their composition occur during brain development, maturation, aging and due to pathological states such a neurodegeneration [177]. Gangliosides are fragile molecules and are easily fragmented during the MALDI ionization process $[56,178]$, 
although in some cases this can be minimised by adjustment of the source pressure [58, 179] Successful MALDI imaging of gangliosides was in the past achieved mostly through the use of specialized matrices typically dissolved in elaborate solvent mixtures $[56,180,181]$. However, the referred publications do not address imaging of other lipid species, so it is unknown if or how much the specialized matrix types influence the ionization of other lipid classes.

The ionization process in MALDI typically occurs in a vacuum chamber. The need of a vacuum-based environment brings along several disadvantages. Perhaps most evidently, it is the need for a sample preparation to ensure a sample's vacuum compatibility. MALDI further requires deposition of a matrix on top of the sample surface. The deposition of a matrix can also limit resolution, sensitivity and reproducibility [182]. The presence of a sample in vacuum further implies a loss of volatile compounds from the sample surface.

Desorption electrospray ionization (DESI) was one of the first ambient MS ionization techniques described [183]. Since its introduction in 2004 it has been gathering momentum as a complementary MSI technique to the more traditional MALDI approach. It was shown to be especially beneficial for the analysis of metabolites and lipids in tissue samples [50]. DESI imaging experiments require no sample preparation as desorption and ionization are initiated by charged droplets impacting the tissue surface directly $[172,183]$. The impact of the ionization probe has low energy, thus complex lipids can be observed. Due to the electrospray character of DESI, multiply charged ions are often present in the spectra [172].

The nature of MSI does not allow for incorporation of a chromatographic separation step prior to an imaging experiment. Hence, MSI information is very complex and typically requires employment of a multivariate data analysis. Ion mobility separation (IMS) is an electrophoretic separation method based on collisional cross-section differences between ions. The separation results from the interaction of ionized molecules with molecules of neutral gas such as nitrogen as they travel through electrostatic field of an ion mobility cell. Eventually, the molecules are separated based on their charge, size and shape. MALDI-MSI coupled to IMS has demonstrated significant utility over the last decade for the separation of matrix/background and tissue endogenous ion species (e.g. lipids) in the gas phase [30, 41]. IMS enables separation of nominally isobaric species that cannot be resolved by time-of-flight mass spectrometers [30, 184].

In this study, we report for the first time on the employment of DESI coupled IMS for the analysis of lipid species in the murine brain. We contrast the DESI-IMS results with MALDI-IMS performed on the same ion mobility enabled instrument. When the intermediate vacuum MALDI source was in operation, a solid-state diodepumped Nd:YAG laser with a repetition rate of $1 \mathrm{kHz}$ was used. When the DESI stage was mounted, the MALDI source was removed and the electrospray inlet block 
was installed along with an appropriate inlet capillary. The parameters for the ion mobility and mass analyzer were kept constant between the two techniques. While MALDI coupled to IMS enabled separation of background from the tissue, DESI data featured a number of tissue related trendlines. We revealed that DESI enables imaging of fragile lipid species directly from the murine brain tissue. Gangliosides were separated from the rest of the lipid classes using ion mobility. We observed and imaged intact poly-sialylated gangliosides as multiply charged ions using DESI-IMS directly from the murine brain tissue. We additionally show that complementary lipid information is reached under particular conditions when DESI is compared to MALDI imaging.

\subsection{Methods}

Tissue samples. Animal experiments were conducted in accordance with German law (in congruence with 86/609/EEC) for the use of laboratory animals and approved by the local animal welfare committee at the Johannes Gutenberg University Mainz. Wild type mice were perfused with cold ammonium acetate $(50 \mathrm{mM}, \mathrm{pH} 7.3)$ in a $4^{\circ} \mathrm{C}$ room to prevent lipid degradation [33]. Brains were rapidly removed and frozen on dry ice and kept at $-80{ }^{\circ} \mathrm{C}$ until further processing. $20 \mu \mathrm{m}$ thin tissue sections were obtained using cryostat (CM 3050 S; Leica Microsystems, Nussloch, Germany). The sections were thaw mounted onto indium tin oxide covered glass slides (Delta Technologies, Loveland, CO, USA). The cut tissue samples were stored at $-80{ }^{\circ} \mathrm{C}$ until further analysis.

Tissue preparation for mass spectrometry imaging. Prior to MSI the tissue sections were placed into a vacuum desiccator for 15 minutes. For MALDI experiments matrix solutions were applied on top of the brain sections using SunCollect (SunChrom, Friedrichsdorf, Germany) spraying device. 20 layers of 5 $\mathrm{mg} / \mathrm{mL}$ of CHCA dissolved in acetonitrile/water $(7: 3, \mathrm{v} / \mathrm{v})$ with $0.1 \%$ addition of trifluoroacetic acid were applied for the positive mode experiments. For negative ionisation mode, 25 layers of $5 \mathrm{mg} / \mathrm{mL}$ 9-aminoacridine (9-AA) in acetonitrile/water $(1: 1, \mathrm{v} / \mathrm{v})$ solution were sprayed. The protocol for matrix application was as follows: The first layer was sprayed at $10 \mu \mathrm{L} / \mathrm{min}$ to seed the tissue section with small crystals of matrix. The second layer was sprayed at $15 \mu \mathrm{L} / \mathrm{min}$. The remaining layers were each sprayed at $20 \mu \mathrm{L} / \mathrm{min}$. The z-dimension was set to $26 \mathrm{~mm}$. For DESI imaging, no additional sample preparation was needed and tissue sections adjacent to those used in MALDI experiments were used "as is".

Instrumentation and data acquisition. Both MALDI and DESI-IMS-MSI experiments were carried out using HDMS SYNAPTTM G2-Si mass spectrometer with Tri-Wave technology (Waters Corporation, Manchester, UK). The DESI source from Prosolia (Indianapolis, USA) incorporated an electrospray probe and was 
utilized as an imaging technique by rastering a sample surface under the spray using a high precision XY stage.

All MSI experiments data were acquired in the "Resolution MS mode" in the mass range between 50 and 1,150 Da. Mass calibration was performed using red phosphorus cluster ions for MALDI imaging experiments and sodium formate clusters for DESI imaging experiments $[185,186]$. MALDI experiments were carried out using a Nd:YAG laser operating at $355 \mathrm{~nm}$. Laser pulse duration was $2 \mathrm{~ns}$ at a fixed repetition rate of $1 \mathrm{kHz}$. MALDI-IMS-MSI data were acquired by irradiating each pixel with 1,000 laser shots. The distance in the $\mathrm{X}$ and $\mathrm{Y}$ direction between each pixel was $100 \mu \mathrm{m}$. DESI experiments were carried out using a mixture of methanol and water $(9: 1 \mathrm{v} / \mathrm{v})$ as a spray solvent and delivered at the flow rate of $4 \mu \mathrm{L} / \mathrm{min}$ using a Harvard syringe pump, with an applied voltage of $4 \mathrm{kV}$. Nitrogen gas was delivered at 7 Bar from an external gas bottle. The sections were scanned in horizontal line at a speed of $100 \mu \mathrm{m} / \mathrm{s}$ with the mass spectrometer collecting mass spectrum every second. Rows were separated by a vertical step of $100 \mu \mathrm{m}$.

The ion mobility separation settings were consistent between the DESI and MALDI imaging experiments. The parameters were set at the following values: pressure in the IMS cell $3.13 \mathrm{mBar}$, wave height $40 \mathrm{~V}$, trap DC bias $60 \mathrm{~V}$, and wave velocity starting at $1,000 \mathrm{~m} / \mathrm{s}$ and finishing $300 \mathrm{~m} / \mathrm{s}$.

Data analysis. All software for data analysis was provided by Waters (Waters Corporation, Manchester, UK). All MSI data were processed using High Definition Imaging (HDI) software. Raw continuum data from each MSI dataset was at first combined into a single mass spectrum, which was subsequently used for peak picking. The peak picking was performed using Apex3D algorithm allowing for each detected component to have an $\mathrm{m} / \mathrm{z}$ and the associated drift time. 2,000 of most intensive peaks were included in the final peak lists. MS images were reconstructed using HDI software. Ion mobility data were explored using DriftScope software, where continuum data was displayed as a two-dimensional plot with $\mathrm{m} / \mathrm{z}$ range in the $\mathrm{Y}$ axis and drift time range in $\mathrm{X}$-axis. Spectral information was investigated using MassLynx.

Lipid identification. The identification of the reported lipid species was based on tandem mass spectrometry experiments and on a search within the LipidMaps database [20]. The tandem mass spectrometry experiments were performed on the same MS instrument, using both, DESI and MALDI source. A lipid ion precursor was selected within a 2 Da selection window and fragmented in the trap cell. For the ion mobility including tandem MS experiments, the precursor ions were fragmented in transfer cell, i.e. after the IMS cell. Fragment peaks were consulted with LipidMaps and literature $[41,51]$. 


\subsection{Results and Discussion}

Spectral information. Figure 3.1a contrasts combined negative mode mass spectra of imaging experiments performed by MALDI and DESI on a mouse brain. Both spectra are dominated by GPL in the mass range between 700 and $900 \mathrm{Da}$. In our experiments, MALDI ionization of ST, i.e. ceramide species with a sulphated sugar moiety, (e.g. $\mathrm{m} / \mathrm{z}$ 888.6) is superior when compared to DESI. DESI spectrum, on the contrary, shows intensive signal related to fatty acids (FA) in the low mass range (250-350 Da). Next to FA, DESI spectrum shows also several specific peaks in the "lysolipid range" (400-600 Da). Due to the character of DESI, the technique produces multiply charged ions. Doubly and triply charged species dominate the negative DESI spectrum in the mass range above $\mathrm{m} / z 900$.

While the negative MALDI mass spectrum does not excessively suffer from the presence of matrix (main matrix peak observed at $\mathrm{m} / \mathrm{z} 193.1$ corresponds to deprotonated 9-AA), MALDI spectrum in positive mode contains a substantial number of matrix peaks and clustered matrix peaks. Peaks marked with an asterisk on Figure $3.1 \mathrm{~b}$ were commonly observed and reported in literature as CHCA clusters [187-189]. The clustered matrix peaks interfere with the lipid signal in the mass range of 700-900 Da, making the spectrum complicated. 


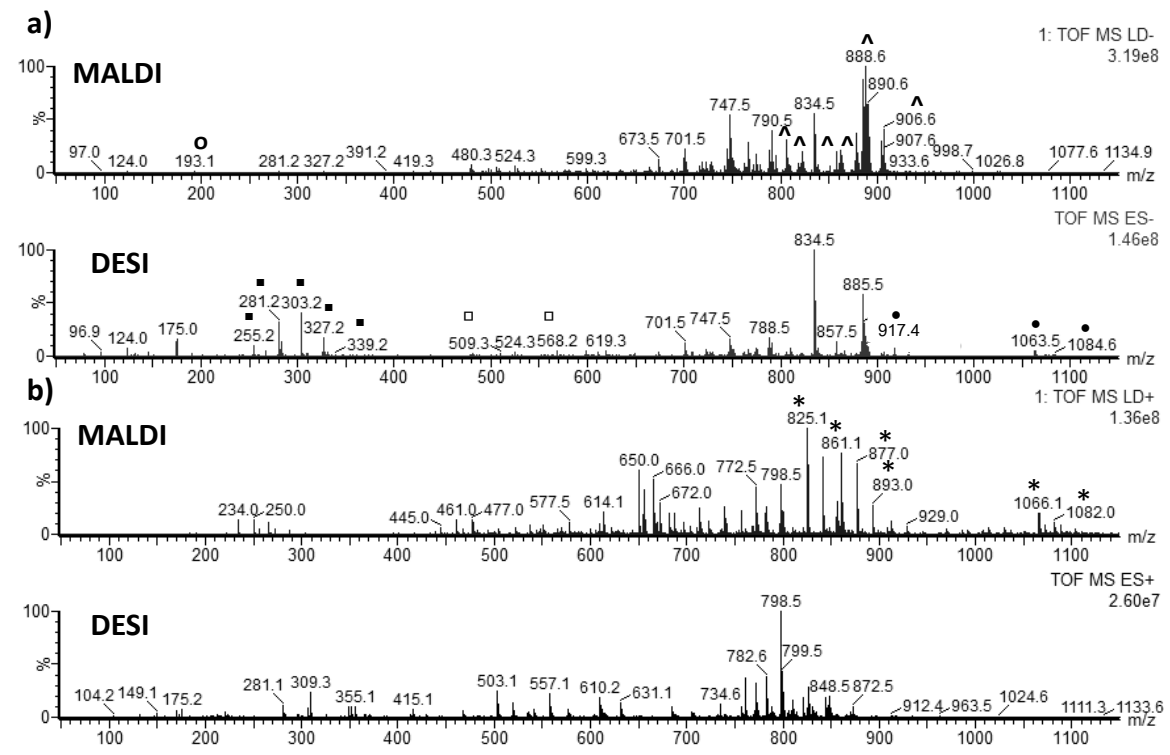

Figure 3.1 Representative a) negative and b) positive total ion mass spectra of MALDI and DESI imaging experiments performed on a mouse brain without ion mobility separation. a) Both MALDI and DESI ionized the glycerophospholipids (e.g. $\mathrm{m} / \mathrm{z}$ 834.5). While MALDI was superior for the ionization of sulphatides, DESI showed a superb efficiency for the ionization of fatty acids. DESI also featured several unique peaks in the lysolipids mass range (450-600 Da). Multiply charged species were observed in negative mode DESI spectrum. b) Positive MALDI spectrum suffers from the presence of matrix clusters which make the lipid region unclear. Legend: o matrix 9-AA, ^ sulphatides, - fatty acids, lysolipids, • multiply charged ions, * matrix clusters (CHCA).

Ion mobility separation. The spectral information presented in Figure 3.1 is very complex since, among others, MALDI matrix peaks and tissue related peaks are mixed up in single combined spectra. IMS has the ability to separate ions based on their charge, shape and size, or in other words, based on their collisional crosssections. As a result, it can quickly separate background signal (matrix peaks, chemical noise etc.) [190] from endogenous tissue molecules [41]. Ion mobility information is typically displayed in a form of a two dimensional (2D) diagram, in which $\mathrm{m} / \mathrm{z}$ is plotted over the drift time [184]. IMS "pre-clusters" particular classes of molecules by sorting them into separate trendlines, which are clearly observed in the 2D diagrams. The spectral information can be further extracted from each trendline separately, thus ensuring distinction of various molecular classes. This is demonstrated in Figure 3.2. which shows the 2D-plot $\mathrm{m} / z$ vs. drift time (referred to from now on as a $m / z$-drift time plot) of our MALDI-IMS imaging experiment performed on a mouse brain section in negative mode. The plot contains two nested 
trendlines out of which the spectral information can be extracted. This step on itself, however, does not allow for conclusions to be drawn on the spatial distribution of the molecules. MSI includes the spatial information, although it results in an increased data complexity. Combing IMS and MSI in a single experiment simplifies and eases MSI data interpretation without compromising the information bounty. Figure $3.2 \mathrm{~d}$ shows that MALDI-IMS-MSI experiment allows for separation of matrix ions from the tissue related molecules.
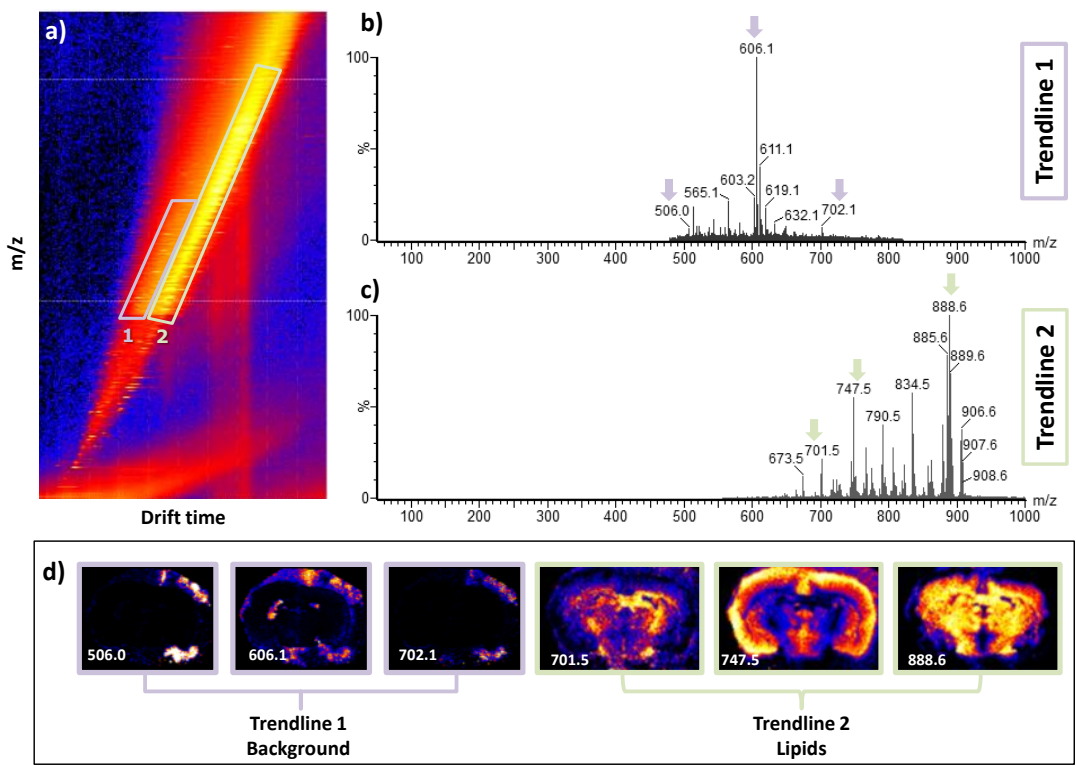

Figure 3.2 MALDI-IMS-MSI of the mouse brain in negative mode. a) Two trendlines can be seen on the $\mathrm{m} / z$-drift time plot. $\boldsymbol{b})$ and $\boldsymbol{c}$ ) The spectral information can be extracted from each trendline separately, thus allowing for distinction of different molecular species. d) The imaging modality allows for conclusions to be drawn on the spatial distribution of the molecules from the particular trendlines. It can be seen, that trendline 1 corresponds to the background, whereas trendline 2 consists of tissue related molecules.

Figure 3.3a shows an overlay of MALDI and DESI $\mathrm{m} / z$-drift time plots in negative mode. The DESI $\mathrm{m} / \mathrm{z}$-drift time plot, unexpectedly, reveals a number of trendlines. Importantly, DESI trendlines correspond exclusively to tissue related molecules as no matrix is applied. Figure 3.3b shows a zoom-in view of the $m / z$-drift time plots in the mass range of 700-900 Da (GPL and ST range). The MALDI plot shows multiple peaks related to the background within this mass range. DESI, on the contrary, contains no background peaks and the "lipid trendline" consist exclusively of lipid species. 


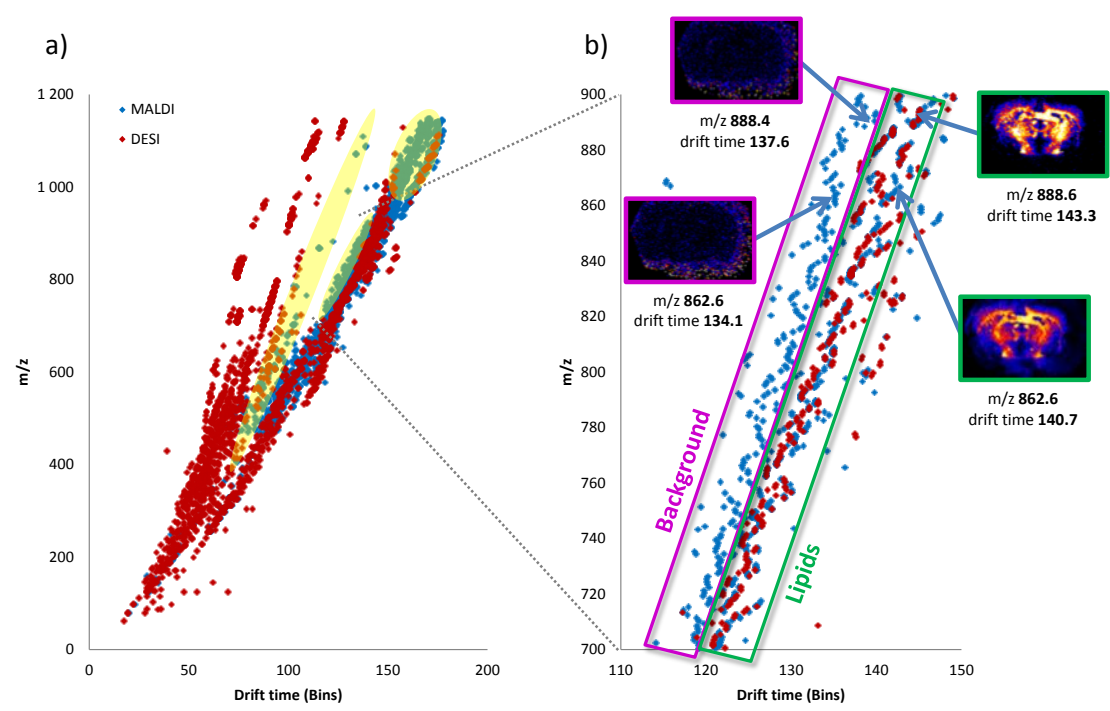

Figure 3.3 Comparison of negative DESI and MALDI $2 D$-plot $\mathrm{m} / \mathrm{z}$ vs. drift time. a) DESI (red marks) offers complementary information on various molecular species when compared to MALDI (blue marks). The yellow areas mark background related (matrix, chemical noise etc.) MALDI peaks. b) A faster trendline corresponding to background peaks was observed in the MALDI plot in the mass range 700-900 Da. For example sulphatides at m/z 888.7 and $\mathrm{m} / \mathrm{z}$ 862.6 and with the drift time values above 140 bins clearly fall into the slower "lipid trendline" (green frame). Ions at the nominally same mass-to-charge ratio $(\mathrm{m} / \mathrm{z} 888.4$ and $\mathrm{m} / \mathrm{z}$, 862.6) with a shorter drift time below 140 bins belong to the "background trendline" (purple frame). DESI plot, on the contrary, does not contain any matrix related peaks, hence the lipid range (700-900 Da) consists exclusively of lipid peaks.

Figure 3.4 shows a detailed overview of the DESI $\mathrm{m} / \mathrm{z}$-drift time plots in both ionization modes. While the negative ion mode (Figure 3.4a), discussed in the next section, features a bounty of trendlines, the positive ion mode (Figure 3.4b) is less complex with only two nested trendlines. The "negative ion mode DESI trendlines" correspond either to different molecular species or to their different charge states. We inspected the particular trendlines and the spatial distribution of the corresponding molecular species in more detail. Our findings are summarized in the following section.

Negative mode. As singly charged species, DESI $m / z$-drift time plot featured FA and lysolipids, both falling into one trendline (trendline 1 in Figure 3.4a). Lysolipids are created in the tissue mostly as products of enzymatic degradation of intact phospholipids, hence their abundance in a healthy tissue is fairly low [191]. GPL were also observed as singly charged ions with DESI (trendline 2 in Figure 3.4a). GPL are the main category of lipids commonly analyzed with MALDI along with ST. 

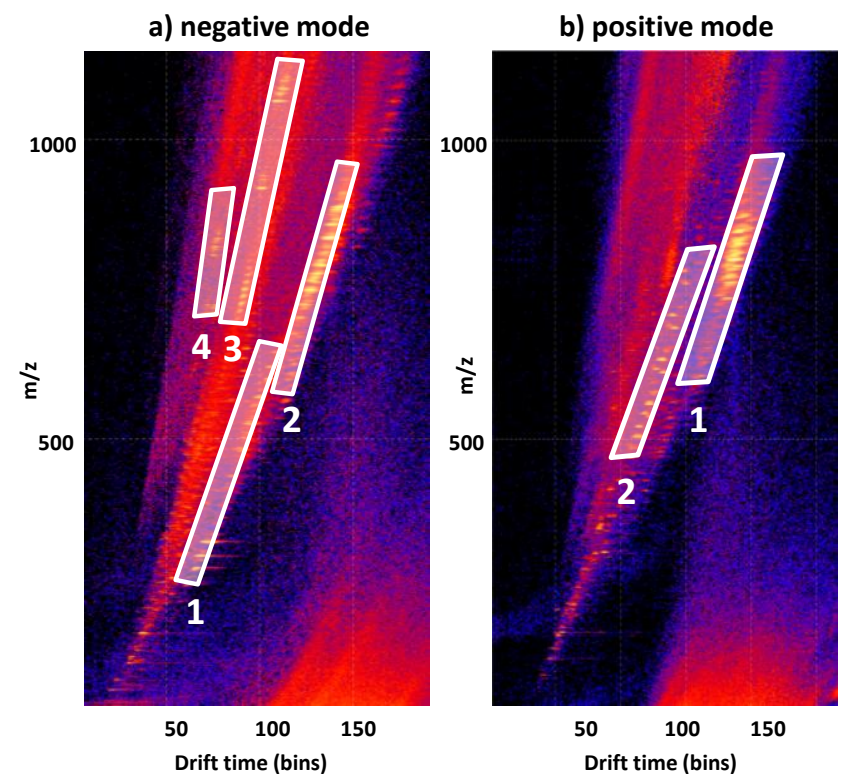

Figure 3.4 2D-plots $\mathrm{m} / \mathrm{z}$ vs. drift time and overview of DESI trendlines in negative and positive ionization mode. a) In negative mode the particular trendlines correspond to different molecular classes or different charge states. $1>$ singly charged fatty acids and lysolipids, $2>$ singly charged glycerophospholipids, $3>$ doubly charged ceramide-based lipids and gangliosides, 4 > triply charged gangliosides. b) Two trendlines were observed in positive ionization mode. $1>$ phosphocholines and sphingomyelines lipids and $2>$ siloxanes (contamination of the electrospray).

\section{Box 2. Nomenclature of gangliosides}

Gangliosides are glycolipids with a very complex chemical structure. Their backbone is created by a di- (d) or tri- (t) hydroxy sphingoid base, with variable composition regarding the fatty acids residues. A mono- to poly-sialylated oligosaccharide chain of variable length is attached to the ceramide portion of the molecule. Similarly, the nomenclature of gangliosides is somewhat complicated: Letters (M, D, T, and Q) refer to a number of sialic acids present in a ganglioside structure. Numbers used in the nomenclature refer to the position of a ganglioside on a TLC plate. The higher the number, the later the ganglioside appears on a thin layer chromatogram. Thus, the longer the carbohydrate chain. GM1, for example, stands for a ganglioside with one sialic acid and the longest carbohydrate chain composed of four sugar molecules.

MALDI-MSI of GPL is well established and documented in the literature. However, MALDI imaging of fragile complex lipids, namely gangliosides, is challenging, due to fragmentation of the oligosaccharide chain and losses of sialic 
acid groups. While GD gangliosides are the predominant ganglioside species in mouse brain, MALDI shows an artificially high signal of GM species due to the fragmentation. During electrospray ionization, multiply sialylated gangliosides are observed as multiply charged ions [177]. Richards et al. previously reported on MSI of gangliosides using laserspray ionization inlet (LSII) [178]. Although the group described a successful analysis of intact ganglioside standards (ranging from GM1 to GQ1 species), only GD1 ganglioside was imaged as a doubly deprotonated ion in the mouse brain. Moreover, despite being an atmospheric pressure imaging technique, a successful LSII experiment relies on the presence of matrix on top of a sample surface. Thus, all matrix related drawbacks, as described previously with respect to MALDI, apply also to LSII imaging. Wiseman et al. reported on detection of ganglioside species from murine brain using DESI [192]. However, the group detected gangliosides from a planar chromatography media after the lipids were eluted from a mouse brain section. The analysis of gangliosides directly from the tissue was not successful under the reported conditions.

Our DESI experiments enabled us to observe intact poly-sialylated gangliosides as doubly and triply deprotonated species directly on the brain tissue surface. Gangliosides were separated into two distinct trendlines on the DESI $\mathrm{m} / \mathrm{z}$-drift time plot according to their charge state. (See trendline 3 and 4, respectively, in Figure 3.4a). GM gangliosides, i.e. molecules with one highly acidic site, naturally favour only singly deprotonated species [193]. We found doubly deprotonated GD1 and GT1 species. As triply deprotonated, we observed GT1 and GQ1 species, including their acetylated versions. This represents a major breakthrough in the direct detection of intact gangliosides from tissue, as the acetylated versions and GQ1 species in general are present in the brain tissue in a particularly low concentration [178]. So far, to the knowledge of the authors, they have not been reported during a MSI experiment. The spectral information corresponding to the respective trendlines is shown in Figure $3.5 \mathrm{a}$ and $\mathrm{b}$. 

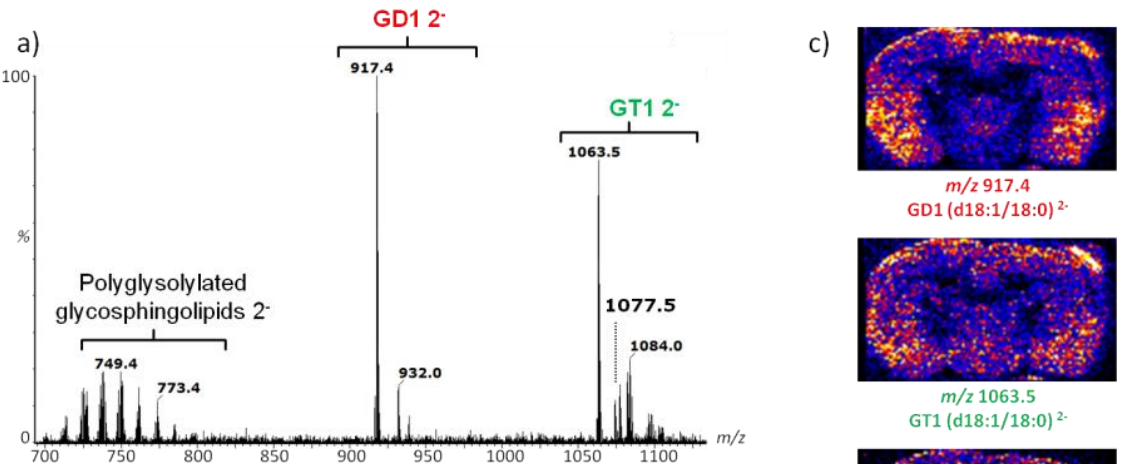

GD1 (d18:1/18:0) $)^{2}$
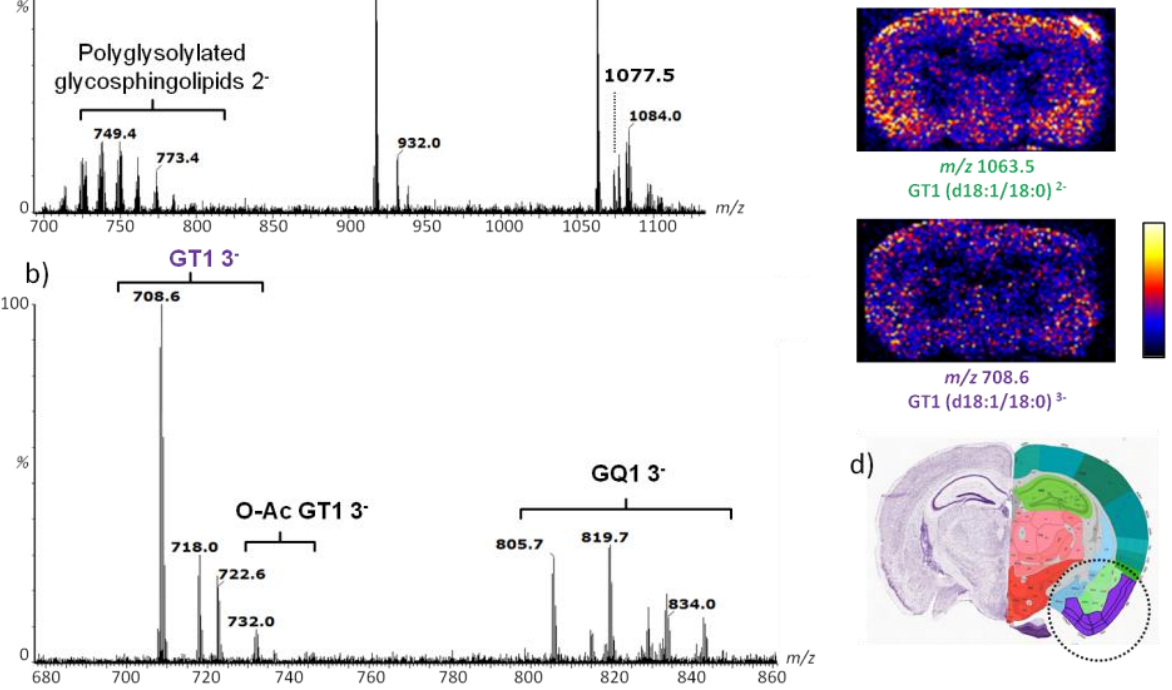

Figure 3.5. DESI images gangliosides as doubly and triply deprotonated ions. a) Spectrum of negative trendline 3 (see Figure 3.4a) containing doubly deprotonated lipid species identified as GDI and GT1 gangliosides. b) Spectrum of triply charged gangliosides (negative trendline 4 in Figure 3.4a) identified as GT1 and GQ1 gangliosides and their corresponding acetylated versions. The tentative identification was based on MSMS experiments and on comparison with the available literature. See the main text and Table 1 for more information on the lipids assignment. c) DESI MS images of the most intensive ganglioside species. GD1b (d18:1/18:0) at $\mathrm{m} / \mathrm{z} .917 .4$ was linked to the cortex, in particular to the olfactory areas of cortex as manually compared to the reference section from the Allen Brain Atlas shown in d). Olfactory area is shown in a violet colour within the dotted circle.

Our identification of gangliosides was based on tandem mass spectra and search in LipidMaps [20]. An example of a recorded on-tissue MSMS spectrum of a ganglioside species identified as GD1b (d18:1/18:0) and observed at $\mathrm{m} / \mathrm{z} 917.4$ is included in Figure 3.6. A dehydrated sialic acid (NeuAc) peak is present at $\mathrm{m} / \mathrm{z}, 290.1$ $\left[\mathrm{NeuAc}-\mathrm{H}_{2} \mathrm{O}^{-}\right]$. During a tandem mass spectrometry experiment of a doubly charged ganglioside, singly charged species are produced as a result of a loss of one of the charged sialic acid molecules. That explains the presence of $\mathrm{m} / \mathrm{z} 1545.9$ in the MSMS spectrum. This singly charged ion represents the loss of a charged sialic acid from the $[\mathrm{M}-2 \mathrm{H}]^{2-}$ species. We further observed a series of sugar losses typical for gangliosides. These singly charged ions are created by the loss of both sialic acids 
and a transfer of the negative charge within the molecule. The ion at $\mathrm{m} / \mathrm{z}, 888.7$ represents ceramide (d18:1/18:0) with attached monosaccharide units. The ion at $\mathrm{m} / \mathrm{z}$ 726.3 represents further loss of galactose. Finally, singly charged ceramide (d18:1/18:0) ion is present at $m / z$ 564.5. The presence of a singly charged dehydrated sialic acid dimer at $m / z 581.3$ suggest the presence of GD1b isomer, wherein the two molecules of sialic acid are attached to each other on a single sugar unit. Conversely, in a molecule of the GD1a isomer, the two sialic acid molecules are attached to two different sugar units, hence the referred dimer cannot be formed upon GD1a fragmentation.

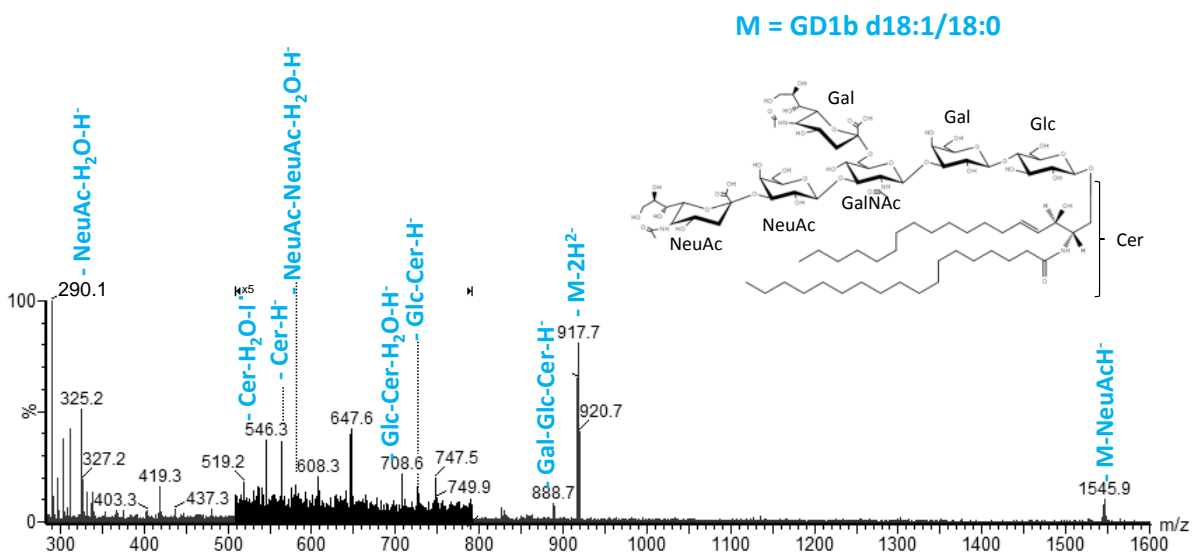

Figure 3.6 Tandem mass spectrum of $\mathrm{m} / \mathrm{z} 917.4$ performed on the brain tissue section. The doubly charged ion was identified as ganglioside GDIb (d18:1/18:0). Consecutive sugar losses are observed at m/z 888.7 (Gal-Glc-Cer-H $\left.{ }^{-}\right), \mathrm{m} / z .726 .6\left(\mathrm{Glc}-\mathrm{Cer}-\mathrm{H}^{-}\right)$and $\mathrm{m} / \mathrm{z} .564 .3\left(\mathrm{Cer}-\mathrm{H}^{-}\right)$. The ions at $\mathrm{m} / \mathrm{z}, 708.6$ and $\mathrm{m} / \mathrm{z}, 546.3$ correspond to a loss of water molecule from the latter two ions, respectively. Dehydrated sialic acid is present at $\mathrm{m} / \mathrm{z}$ 290.1.The ion at $\mathrm{m} / \mathrm{z}, 1545.9$ represents the loss of a charged sialic acid from the $[\mathrm{M}-2 \mathrm{H}]^{2-}$ species. Finally, the presence of a singly charged dehydrated sialic acid dimer at $\mathrm{m} / \mathrm{z}, 581.3$ (even though its intensity is low) suggest the presence of GDIb isomer. Glu $=$ glucose, Gal = galactose, Cer = ceramide, NeuAc $=$ sialic acid, GalNAc $=N$-acetylgalactosamin

However, in most cases the sensitivity of our experiments was not sufficient to perform an on-tissue tandem MS experiment. In such cases, the tentative identification was based on the available literature [177, 178, 192-194]. $\mathrm{m} / \mathrm{z} 917.4$ and $m / z$ 932.0 were identified as GD1 (d18:1/18:0) and GD1 (d18:1/20:0), respectively. The doubly charged ion at $m / z \quad 1063.5$ was assigned as GT1 (d18:1/18:0). During electrospray experiments, gangliosides also tend to create deprotonated sodiated adducts [177]. For example, $\mathrm{m} / \mathrm{z}$ 1084.0, observed as a doubly deprotonated species, was tentatively identified as GT1 (d18:1/18:0) molecule observed as $[\mathrm{M}+2 \mathrm{Na}-4 \mathrm{H}]^{2-}$. This particular GT1 ganglioside was also found as a 
triply charged ion at $m / z, 708.6$, together with its low abundant acetylated version at $m / z$ 722.6. In lower abundance, GT1 (d18:1/20:0) was also observed as a doubly $(\mathrm{m} / \mathrm{z}, 1077.5)$ and triply deprotonated ion $(\mathrm{m} / \mathrm{z}, 718.0)$, including its acetylated version $(\mathrm{m} / \mathrm{z}$ 732.0). GQ1 species were also observed, albeit in a low abundance. $\mathrm{m} / \mathrm{z} 805.7$ tentatively corresponds to GQ1 (d18:1/18:0), and $m / z, 819.7$ to its acetylated version. Table 3.1 summarizes the gangliosides detected during our DESI-IMS-MSI experiments and proposed identification.

\begin{tabular}{llcl}
$\boldsymbol{m} / \mathbf{z}$ & lon ID & \multicolumn{1}{l}{ Charge state } & Identification \\
\hline 917.4 & $\mathrm{M}-2 \mathrm{H}$ & $2-$ & GD1 (18:1/18:0) \\
\hline 932.0 & $\mathrm{M}-2 \mathrm{H}$ & $2-$ & $\mathrm{GD1}(\mathbf{2 0 : 1 / 1 8 : 0 )}$ \\
\hline 1063.5 & $\mathrm{M}-2 \mathrm{H}$ & $2-$ & $\mathrm{GT1}(\mathbf{1 8 : 1 / 1 8 : 0 )}$ \\
1084.0 & $\mathrm{M}+2 \mathrm{Na}-4 \mathrm{H}$ & $2-$ & \\
708.6 & $\mathrm{M}-3 \mathrm{H}$ & $3-$ & \\
722.6 & $\mathrm{M}-3 \mathrm{H}$ & $3-$ & $\mathrm{O}-\mathrm{AC}$ \\
\hline 1077.5 & $\mathrm{M}-2 \mathrm{H}$ & $2-$ & $\mathrm{GT1}(\mathbf{1 8 : 1 / 2 0 : 0 )}$ \\
718.0 & $\mathrm{M}-3 \mathrm{H}$ & $3-$ & \\
732.0 & $\mathrm{M}-3 \mathrm{H}$ & $3-$ & O-AC \\
\hline 805.7 & $\mathrm{M}-3 \mathrm{H}$ & $3-$ & GQ1 (18:1/18:0) \\
819.7 & $\mathrm{M}-3 \mathrm{H}$ & $3-$ & O-Ac \\
\hline
\end{tabular}

Table 3.1 Gangliosides detected with DESI from the murine brain section and their proposed identification.

Although the intensity of some molecular species was not sufficient to reconstruct a contrast rich MS image, they are clearly visible in the extracted spectra. The most contrast rich images were generated by gangliosides identified as GD1 (d18:1/18:0) and GT1 (d18:1/18:0), observed as doubly charged ions at $\mathrm{m} / \mathrm{z}, 917.4$ and $\mathrm{m} / \mathrm{z}$ 1063.5, respectively. The latter was also imaged as a triply charged ion at $\mathrm{m} / \mathrm{z} .708 .6$, albeit with a lower contrast. The MS images are shown in Figure 3.5c.

The spatial distribution of GD1b (d18:1/18:0) is linked to cortex. This is in agreement with the work of Vajn et al., who mapped the distribution of gangliosides in the murine brain using antibodies and found GD1 species abundantly in the cortex [195]. When manually compared to the corresponding reference mouse brain section from the Allen Brain Atlas [196], the highest abundance of GD1b (d18:1/18:0) was linked to the area roughly corresponding to the olfactory part of the cortex (Figure $3.5 \mathrm{~d})$. Interestingly, Vajn et al. reported relatively high concentrations of GD1 in the olfactory bulb. Although we have to stress our co-localization is only tentative and by 
no means precise, it demonstrates the power of MSI for mapping the gangliosides within the brain with high molecular specificity. (A workflow for comparison of MS images with the Allen Brain Atlas for a precise anatomic localization is introduced in Chapter 5 of this thesis.) The use of antibodies actually does not allow for distinguishing gangliosides with different fatty acid composition. The possibility of imaging gangliosides directly from the tissue surface using DESI thus brings new opportunities to study gangliosides within the brain. This can bring new insights into pathology of ganglioside-metabolism related pathologies, such as the lysosomal storage disorders [197]. Sensitivity of the method can further be improved to enable imaging of all ganglioside species and their acetylated version present in the brain with a high molecular specificity. Next to doubly charged gangliosides, acidic glycosphingolipids were also present in the spectra as doubly charged ions. $\mathrm{m} / \mathrm{z} 749.4$ and $m / z 773.4$ were tentatively assigned as polyglycosylated sphingolipids.

Positive mode. Positive mode IMS showed fewer differences between MALDI and DESI. This is shown in Figure 3.7. DESI showed only two trendlines on the $\mathrm{m} / \mathrm{z}$ drift time plot, as can be also seen in Figure 3.4b. The more intense trendline (number 1) corresponds to singly charged phospholipids. They are the most abundantly ionized lipids in positive mode. The molecules carry a quaternary nitrogen, which makes them "pre-ionized" and easy to be MS analyzed. The phospholipid signal is typically superior to all other tissue lipid signal [31]. Manicke et al. detected SM and PC in both, positive and negative ion mode [198]. In the latter, the species were detected as chlorine adducts. The authors suggest the adducts were formed due to presence of chlorine in the extracellular matrix. We did not detect either of these species in the form of chlorine adducts, possibly due to a different tissue type. 


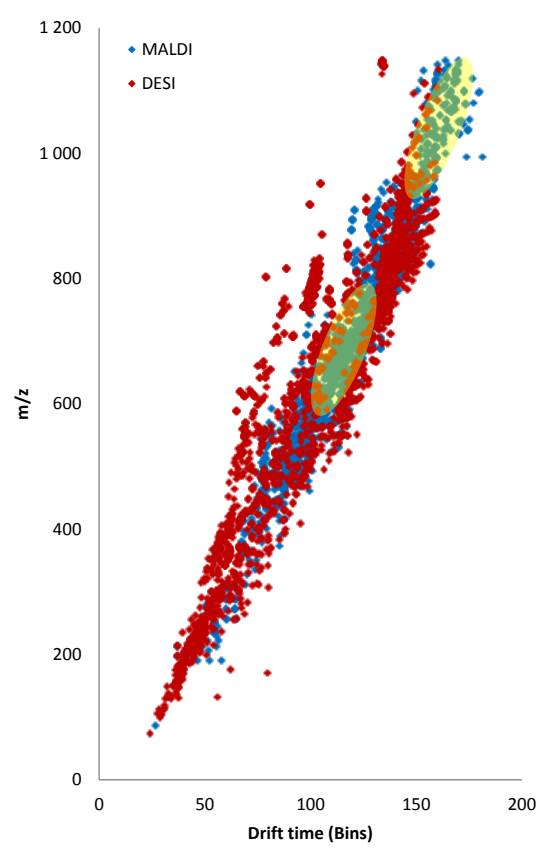

Figure 3.7 Comparison of positive DESI and MALDI 2D-plot m/z vs. drift time. Less difference was observed between DESI (red marks) and MALDI (blue marks) in positive mode. A lower number of trendlines was observed in DESI when compared to the negative mode experiments. The yellow areas highlight matrix clusters observed in MALDI.

We also observed a faster trendline on the positive DESI $\mathrm{m} / \mathrm{z}$-drift time plot (Figure 3.4b), which included $\mathrm{m} / \mathrm{z}$ with spatial distribution related to the outside of the tissue (Figure 3.8) Also the positive mass defect of 0.1 did not suggest lipid species. The structure of the species was elucidated through tandem MS experiments. Major fragments corresponding to siloxane species were observed. Ions at $\mathrm{m} / z, 429.1$ and $\mathrm{m} / \mathrm{z}$ 503.1 correspond to siloxanes $\mathrm{C}_{11} \mathrm{H}_{33} \mathrm{Si}_{6} \mathrm{O}_{6}$ and $\mathrm{C}_{13} \mathrm{H}_{39} \mathrm{Si}_{7} \mathrm{O}_{7}$, respectively, $m / z$ 519.1 correspond to a siloxane $\mathrm{C}_{14} \mathrm{H}_{43} \mathrm{Si}_{7} \mathrm{O}_{7}$. Species at $m / z 610.2$ and $m / z 684.2$ correspond to siloxanes with nitrogen incorporated into the molecules. They were observed as $\mathrm{NH}^{4+}$ species. The two species were identified as $\mathrm{C}_{16} \mathrm{H}_{52} \mathrm{NSi}_{8} \mathrm{O}_{8}$ and $\mathrm{C}_{18} \mathrm{H}_{58} \mathrm{NSi}_{9} \mathrm{O}_{9}$ based on tandem mass spectra. Siloxanes are likely carried in the electrospray stream and are a common contaminant in mass spectrometry. Ion mobility enabled the separation of the pollutants from the rest of the biologically relevant molecules.

However, within the same trendline, we also observed $m / z$ with spatial distribution linked to the tissue, as seen in Figure 3.8, $\mathrm{m} / \mathrm{z}$ 467.1, 541.1, and 557.1. The mass difference of these ions when compared to the siloxanes $(\mathrm{m} / \mathrm{z} 429.1, \mathrm{~m} / \mathrm{z}$ 503.1 and $m / z 519.1$, respectively), was $38 \mathrm{Da}$ and they were identified as potassiated 
siloxanes adducts. The mass difference between $\mathrm{m} / \mathrm{z} 610.1$ (background) and $\mathrm{m} / \mathrm{z} 631.1$ (tissue) and between $\mathrm{m} / \mathrm{z} 684.2$ (background) and $\mathrm{m} / \mathrm{z} 705.1$ (tissue) is 21 Da. It was confirmed that the ions correspond to the respective siloxane species which underwent the loss of $\mathrm{NH}^{4+}$ and are newly potassiated. We suggest that the siloxanes upon the interaction with the tissue react with the potassium. The MS images of the potassiated siloxanes thus reflect the distribution of potassium within the tissue.

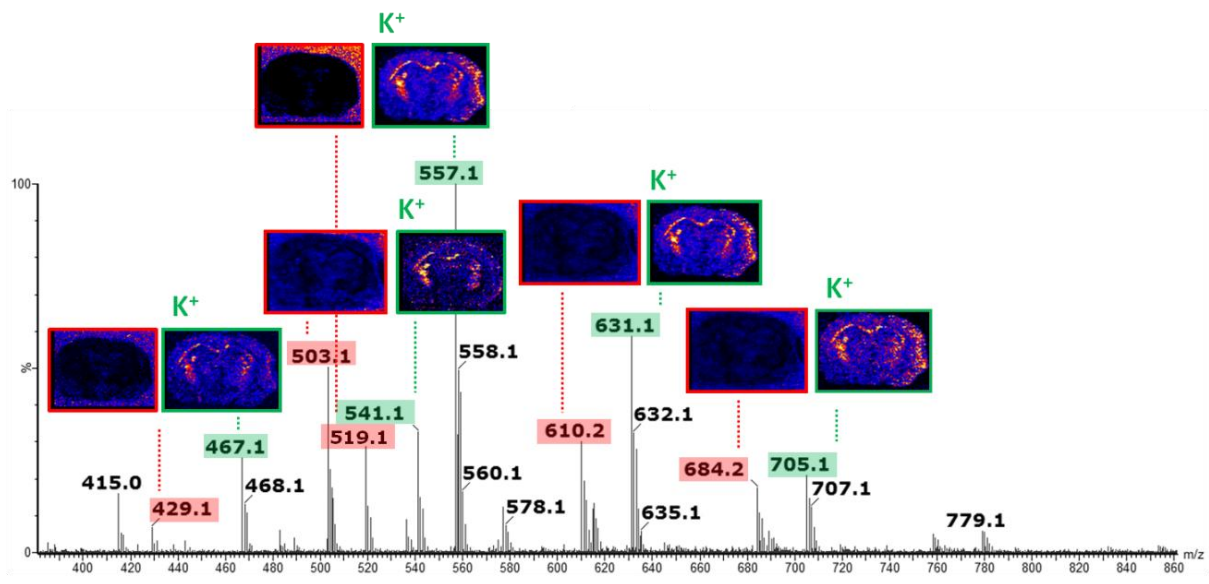

Figure 3.8 Positive mode DESI imaging of siloxanes carried in the stream of the electrospray. Peaks included in trendline number 2 in Figure 3.4b. Siloxanes are shown in red squared images. The species are present only on the outside of the tissue. In green squares are shown potassiated siloxanes. We suggest that siloxanes react with potassium present in the tissue and thus reflect the distribution of potassium within the brain section.

Complementary lipid information. We report DESI and MALDI MSI performed on the same ion mobility enabled instrument. The advantage of using the same instrument with an interchangeable source ensures a high degree of comparability of the IMS results. DESI enabled detection of intact poly-sialylated gangliosides and their acetylated versions directly from the tissue surface under simple experimental conditions. That has been proven especially challenging using MALDI.

Importantly, using DESI, we detected gangliosides next to other common lipid species under the same experimental conditions. This allows us to study the brain lipidome in great molecular detail. Moreover, lipid species that demonstrated lower ionization efficiency in DESI ionized very well under MALDI conditions. Figure 3.9 shows that lipid information obtained with DESI and MALDI under the herein presented conditions is complementary. In negative mode, DESI showed better signal for fatty acids, lysolipids, phosphoglycerols, phosphoserines and in minor for 
phosphatidic acids, while MALDI offered better signal for phosphoethanolamines and sulphatides (note the better image quality of phosphoethanolamine 38:4 as shown in Figure 3.9). Ionization efficiency of phosphoinositols was the same for both techniques. In positive mode, DESI did not yield signal for sphingomyelines (even though their ionization using DESI was reported $[42,199])$, which ionized very well under the MALDI conditions. In contrast, signal for PC was slightly yielded in DESI when compared to MALDI.

Note that each molecular species will have the same drift time for DESI and MALDI due to the identical instrument settings used for both techniques, which further aids the identification. The drift time tolerance is $1 \mathrm{bin}$. In the negative mode, we observed the following trend in the drift time lengths: $\mathrm{PA}<\mathrm{PE}=\mathrm{PG}<\mathrm{PS}<$ $\mathrm{PI}=\mathrm{ST}$. This was in accordance with Jackson et al. who studied lipids using MALDI imaging coupled to IMS [200]. PA have the fastest mobility because only a proton is attached to the phosphate group, PI and ST are the bulkiest molecules with the sugar moiety, hence their drift time is the longest [200]. Table 3.2 summarizes major MSMS fragments of the presented lipids. 


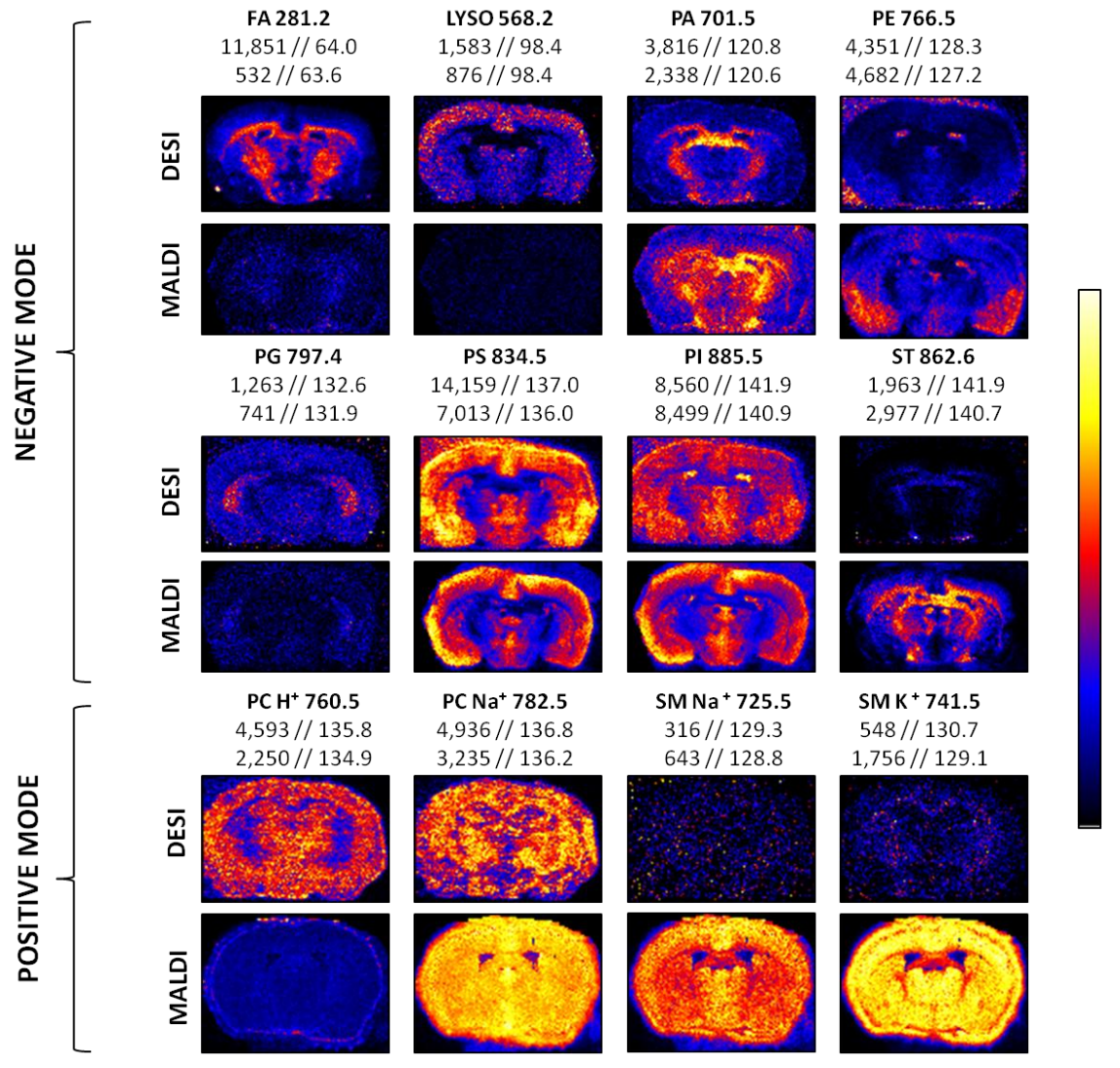

Figure 3.9. Comparison of DESI and MALDI reconstructed MS images of lipid species in negative and positive ionization modes. In negative mode: shown is an example of a fatty acid (FA), a lysolipid (LYSO), all classes of glycerophospholipids: phosphatidic acid (PA), phosphoethanolamine (PE), phosphoglycerol (PG), phosphoserine (PS), and phosphoinositols $(P I)$, and a sulphatide (ST). The images are shown at their highest intensity, the low intensity threshold was kept at zero. In positive mode: shown is an example of phosphocholine (PC) and sphingomyelin (SM). The intensity value is displayed above the images together with the drift time of the particular lipids (intensity // drift time expressed in bins). FA 18:1, LYSO PS 22:6, PA 36:1, PE 38:4, PG 38:4, PS 40:6, PI 38:4, STd18:1/22:0, PC 34:1 and SM 34:1. The lipids identification was based on tandem mass spectrometry experiments and on a search within the LipidMaps database. Results of MSMS experiments are summarized in Table 3.2. We demonstrate complementarity of the lipid information reached by both techniques under the herein reported conditions. In negative mode, DESI showed better ionization efficiency for FA, LYSO, PG, PS and in minor for PA. MALDI was better for imaging of PE and ST. Equal results were reached for PI. In positive mode MALDI demonstrated better ionization efficiency for sphingomyelines, while DESI showed slightly better ionization efficiency for PC. Note that differences in the ionization efficiencies might be observed under different experimental conditions. 


\begin{tabular}{|c|c|c|c|c|}
\hline $\mathrm{m} / \mathrm{z}$ & Fragments & $\begin{array}{l}\text { Lipid } \\
\text { class }\end{array}$ & $\begin{array}{l}\text { Fatty } \\
\text { acyls }\end{array}$ & ID \\
\hline \multicolumn{5}{|c|}{ Negative mode } \\
\hline 568.2 & $152.9,283.2,481.2$ & PS & $\begin{array}{l}22: 6 \\
18: 0\end{array}$ & LPS 22:6 \\
\hline 701.5* & $79.0,153.0,281.2,283.2,417.2,419.2,437.2$ & PA & $\begin{array}{l}18: 1 \\
18: 0\end{array}$ & PA 36:1 \\
\hline 766.5 & $79.0,153.0,283.2,303.2,480.3$ & PE & $\begin{array}{l}20: 4 \\
18: 0\end{array}$ & PE 38:4 \\
\hline 797.4* & $\begin{array}{l}\text { 153.0, 283.2, 303.3, 419.3, } 513.2 \\
79.0,152.9,283.2,327.2,419.2,437.2,463.1\end{array}$ & PG & $\begin{array}{l}20: 4 \\
18: 0\end{array}$ & PG 38:4 \\
\hline 834.5 & $\begin{array}{l}747.4 \\
79.0,153.0,241.0,2832 ., 303.2,419.2,581.2\end{array}$ & PS & $\begin{array}{l}22: 6 \\
18: 0\end{array}$ & PS 40:6 \\
\hline 885.5 & 599.3 & $\mathrm{PI}$ & $\begin{array}{l}20: 4 \\
18: 1\end{array}$ & PI 38:4 \\
\hline $862.6 *$ & $79.0,97.0,153.0,241.0$ & ST & 22:0 & ST d18:1/22:0 \\
\hline \multicolumn{5}{|c|}{ Positive mode } \\
\hline 760.5 & $125.1,184.2$ & PC & $\begin{array}{l}16: 0 \\
18: 1 \\
16: 0\end{array}$ & PC 34:1 \\
\hline 782.5 & $147.1,184.2,599.2,723.7$ & PC & $\begin{array}{l}18: 1 \\
16: 0\end{array}$ & PC $34: 1 \mathrm{Na}+$ \\
\hline 725.5 & $147.1,184.2,469.3,542.6,579.5,601.6$ & SM & $\begin{array}{l}18: 1 \\
16: 0\end{array}$ & $\mathrm{SM} 34: 1 \mathrm{Na}^{+}$ \\
\hline 741.5 & $184.2,163.1,617.6,579.7,558.6$ & SM & $18: 1$ & SM 34:1 $\mathrm{K}^{+}$ \\
\hline
\end{tabular}

Table 3.2 Identification of the lipids presented in Chapter 3. Listed are the observed major fragments of the respective lipid species, which supported the identification. Tandem mass spectrometry experiments were performed as described in the Section 3.2. Note that the position of the fatty acyls may differ, thus the final identification includes a sum of the carbons and double bonds present in a molecule. The MSMS spectra of the peaks highlighted in bold are further shown and discussed. Lipids marked with an asterisk represent signal from more than one molecule. The reported ID corresponds to a molecule with the major contribution to the signal.

Lipid identification. In MS based lipidomics, identification of lipid species is typically performed through an observed fragmentation pattern and comparison of the resulting spectrum with a publicly available database. LipidMaps [20] consortium is a standard database used for MS lipidomics studies.

To identify a glycerophospholipid, peaks indicative for the headgroup and fatty acyls must be present in a spectrum. Theoretically, for every esterified fatty acid in a glycerophospholipid, there should be 3 peaks. One for the anionic fatty acid, and 2 for its neutral loss as a neutral fatty acid and a ketene. Example of an annotated tandem MS spectrum for $\mathrm{m} / \mathrm{z} 834.5$ is shown in Figure 3.10. $\mathrm{m} / \mathrm{z} 152.9$ corresponding to 
dehydrated glycerol-3-phosphate is characteristic for glycerophospholipids. Neutral loss of $87 \mathrm{Da}$ corresponds to the loss of the headgroup molecule (dehydrated serine) and is characteristic for phosphoserines. Ions at $m / z \quad 419.2$ and $m / z 463.1$ are generated as neutral losses of fatty acyls (22:6) and (18:0), respectively. The observed fragmentation pattern led to an unambiguous identification of the molecule as phosphoserine with fatty acyls corresponding to stearic (18:0) and docosahexaenoic acid (22:6). Unfortunately, it is impossible to indicate the position of the fatty acyls, nor the position of the double bonds or potential branching of the acyl chains.

$m / z 834.5[\mathrm{M}-\mathrm{H}]^{-P S}(18: 0 / 22: 6)$

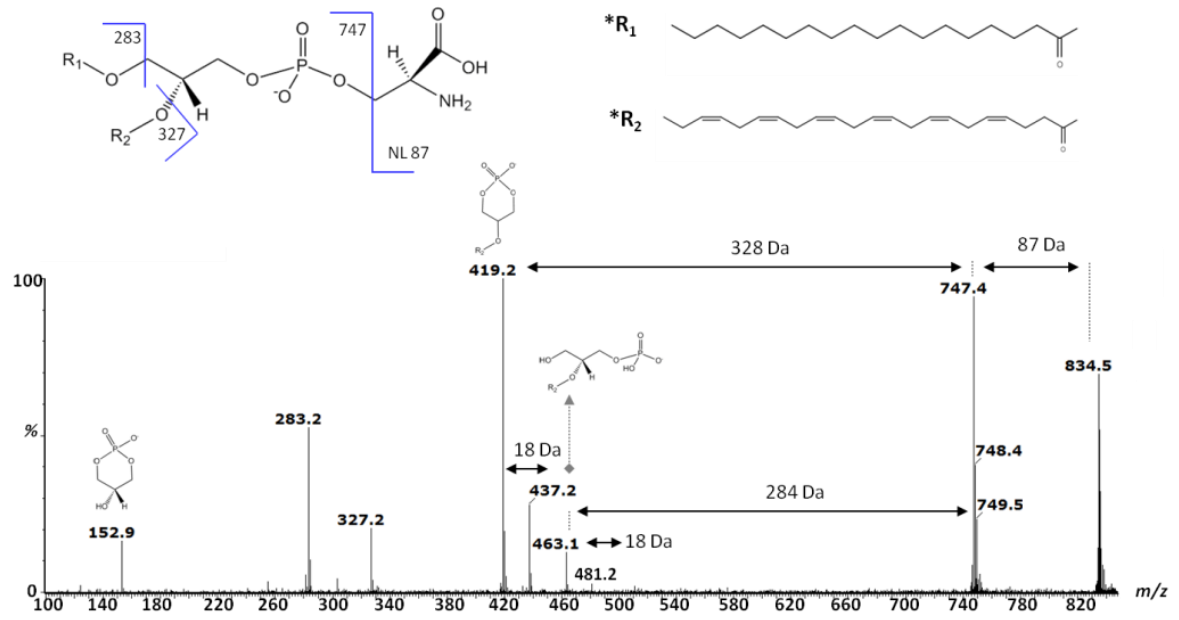

Figure 3.10 Tandem MS spectrum of $\mathrm{m} / \mathrm{z} 834.5$ identified as phosphoserine with stearic (18:0) and docosahexaenoic (22:6) fatty acyls. *Note that the position of $R_{1}$ and $R_{2}$ can be interchanged, as well as the positions of the double bonds. Neutral loss of $87 \mathrm{Da}$ corresponds to the loss of headgroup molecule and is characteristic for phosphoserines. $\mathrm{m} / \mathrm{z} 419.2$ and 463.1 are generated as neutral losses of fatty acyls (22:6) and (18:0), respectively.

The width of a mass window selected for tandem MS experiments presented in this chapter was 2 Da. Narrowing down this range led to a rapid decrease of sensitivity. Although lipids are a structurally broad group of molecules, individual lipid species from the same lipid class can be structurally very similar. As a result, their tandem MS spectra are complicated by the occurrence of isobaric ions. These cannot be separated for a tandem MS experiment based on $\mathrm{m} / \mathrm{z}$. Consequently, fragments of more than a single molecule are sometimes present in the tandem MS spectra.

In our experiments, this was the case for $m / z 701.5, \mathrm{~m} / \mathrm{z} 797.5$ and $\mathrm{m} / \mathrm{z}$ 862.5. Figure 3.11. shows a tandem MS spectrum of $\mathrm{m} / \mathrm{z}$ 701.5. Major fragments 
correspond to stearic (18:0) and oleic (18:1) acid at $\mathrm{m} / \mathrm{z} 283.2$ and $\mathrm{m} / \mathrm{z} \quad 281.2$, respectively. Also neutral losses of the fatty acyls are present at $m / z 417.3$ and $m / z$ 419.3. A peak indicating phosphatidic acid is not present, but further presence of dehydrated glycerol-3-phosphate at $\mathrm{m} / \mathrm{z}, 153.0$ indicates a glycerophopholipds species. After matching these data with LipidMaps database, one ID is assigned: PA (18:1/18:0). However, $m / z 255.2$ corresponding to palmitic (16:0) acid is also present in the fragmentation spectrum. This suggests, that within the isolated window, another lipid species containing palmitoyl was fragmented. After comparison with LipidMaps, we can assume that an isobaric lipids species - PA (16:0/20:1) - is also present in the mass window selected for fragmentation. However, no other peaks confirming its presence are found in the spectrum. For example neutral loss of fatty acid (20:1) with $m / z 309.2$ would be present at $m / z$ 391. Instead of this $m / z$, however, we observed ion at $m / z$ 387.4, which remained unidentified. Taking into account the major fragments led to the proposed identification as presented in Table 3.2.

$m / z 701.5[\mathrm{M}-\mathrm{H}]^{-}$PA $(18: 0 / 18: 1)$
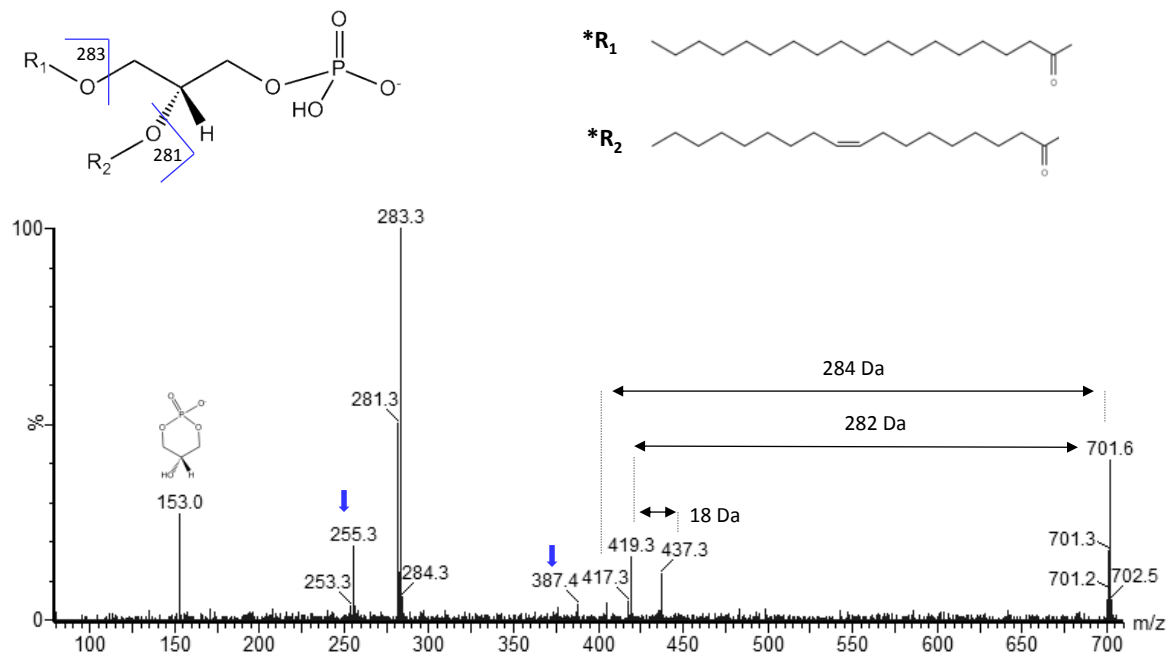

Figure 3.11 Tandem MS spectrum of $\mathrm{m} / \mathrm{z} 701.5$ identified as phosphatidic acid with stearic (18:0) and oleic (18:1) fatty acyls. *Note that the position of $R_{1}$ and $R_{2}$ can be interchanged, as well as the position of the double bond. We see corresponding fatty acyls at $\mathrm{m} / \mathrm{z} 283.2$ and $\mathrm{m} / \mathrm{z} 281.2$ as well as ions resulting from their neutral losses at $\mathrm{m} / \mathrm{z} .417 .3$ and $\mathrm{m} / \mathrm{z} .419 .3 \mathrm{~m} / \mathrm{z}$ 255.3 \{palmitic acid, (16:0)\} suggest a presence of another lipid fragmented within the mass isolation window. $\mathrm{m} / \mathrm{z} 387.4$ remained unidentified.

IMS has the potential to separate isobaric ions based on their collisional cross sections. However, the resolution of current IMS instruments is not high enough to separate lipids of the same class differing in the position of fatty acyls. In the 
literature, we can find a few examples of IMS separating a lipid molecule from an isobaric peptide or a background related molecule [41]. We performed a tandem mass spectrometry experiment of $\mathrm{m} / z, 797.5$ following IMS. The result of its fragmentation is shown in Figure 3.12. Panel a) shows the total ion mobilogram with three peaks (albeit not baseline separated). Panel b) shows a combined MSMS spectrum of all ions without ion mobility separation. Interpretation of such a spectrum does not lead to a clear identification of the precursor ions. Panel c) shows fragments of the slowest ion (highlighted with green mark on panel a). It contains acyls of palmitic $(\mathrm{m} / \mathrm{z}$ 255.2), stearic $(m / z, 283.2)$ and oleic $(m / z, 281.2)$ acid. Peak at $m / z 745.6$ remained unidentified. This shows that still more than one molecular species was fragmented. Although we consider presence of lipid species, we could not asses the identity of the mixture. Panel d) shows fragments of the slower ion identified as phosphoglycerol with stearic (18:0) and arachidonic (20:4) acid.
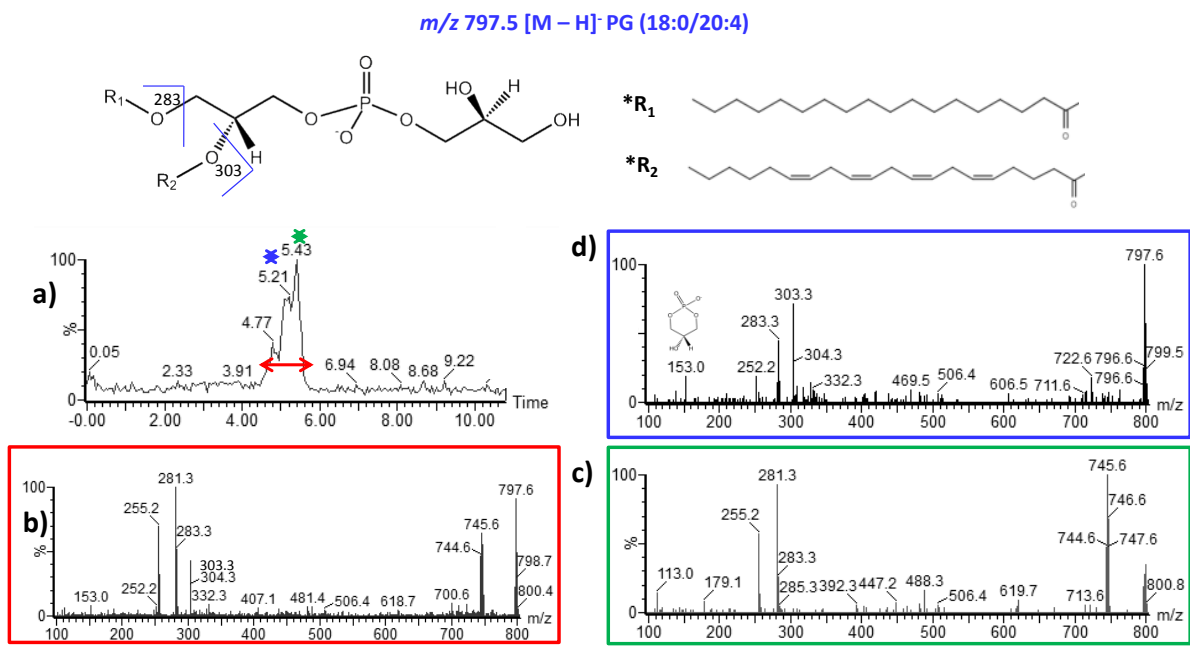

Figure 3.12 Ion mobility separation tandem $M S$ of $m / z$ 797.5. a) Drift time plot showing three peaks (drift time expressed in milliseconds).b)MSMS spectrum of fragments detected from all ions without ion mobility separation. c) Fragments of the slowest ion. Molecular species remained unidentified. d) Fragments of the slower ion identified as phosphoglycerol with stearic (18:0) and arachidonic (20:4) acid. *Note that the position of $R_{1}$ and $R_{2}$ can be interchanged, as well as the positions of the double bonds.

Solvent influence. In our experiments, we achieved complementary lipid information when DESI was compared to MALDI. However, the complementarity can't be explained solely by the nature of the two ionization techniques. As already suggested, there is a room for sample preparation (MALDI) and experimental setup (DESI) adjustments. For example in MALDI, particular classes of lipids will ionize to 
a different extent based on the type of matrix applied. The choice of matrix types for MALDI experiments performed in our study, was guided by the available literature, which showed that 9-AA and CHCA are commonly used for negative and positive MALDI-MSI of lipids, respectively [40, 41, 201]. In the referred studies these MALDI matrices were dissolved in solvent systems of variable composition without evident influence on the type of the extracted lipids. Interestingly, it was shown that lipids can be MALDI imaged while using a solvent-free matrix deposition, such as sublimation [202].

We performed an experiment to study the influence of a solvent system in MALDI on the extraction of lipids. We used 9-AA matrix dissolved in 50\% acetonitrile and in $90 \%$ methanol (for direct comparison to DESI conditions). We applied the solutions on each half of a murine brain section. The application process was identical to the one described in section3.2 Methods. While one of the halves of the section was being sprayed with matrix, the other one was covered with a piece of aluminium foil. Following the matrix application, the tissue section was MALDI imaged in negative mode with instrument parameters set as described previously, without including IMS.

The results of our experiment are shown in Figure 3.13. On one hand, we observed differences for FA whose intensity was higher when methanol was involved and for ST, which were better extracted using acetonitrile. On the other hand, we did not observe better ionization of PE using acetonitrile. This would be expected if we attributed the difference between DESI and MALDI in ionization rates particular lipid classes only to the solvent systems employed. Similarly, ionization of PG was not enhanced in MALDI when using methanol.

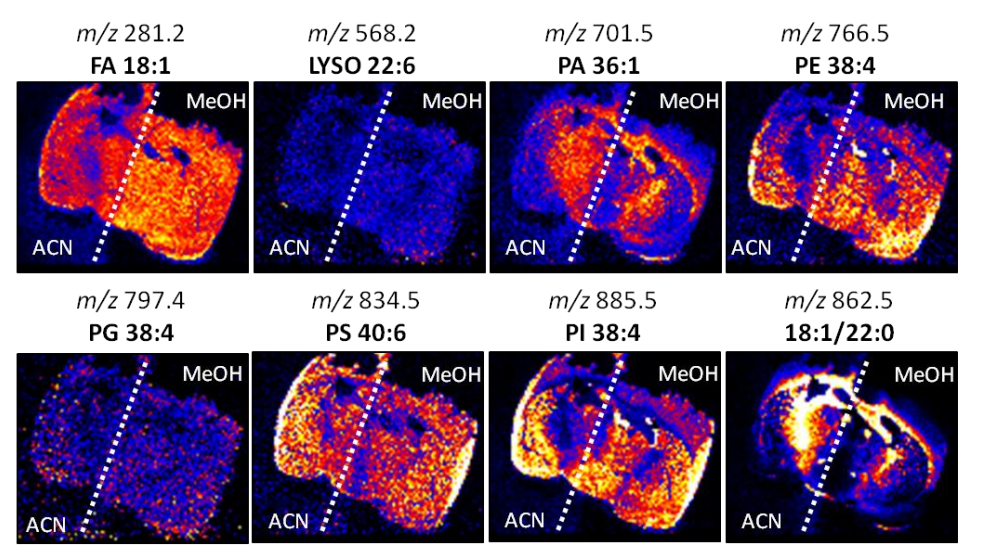

Figure 3.13 Influence of an employed solvent system on extraction of individual lipid classes using MALDI. 9-AA matrix dissolved in 50\% acetonitrile (ACN) and 90\% methanol (MeOH) was applied on each half of the section. The murine brain section was imaged in negative mode. Ionization differences are observed for FA and ST. 
The efficiency of ionization of individual lipid classes in MALDI is a mutual combination of a matrix type and a solvent system employed. The preliminary results of our experiment suggest, that the solvent type has a minor influence. However, it has to be noted, that an indisputable advantage of using higher content of organic solvent is in the finer crystals formed on top of a tissue section. This is especially beneficial for high spatial resolution MSI.

\subsection{Conclusions}

In our work we employed desorption electrospray ionization (DESI) mass spectrometry imaging coupled to ion mobility separation (IMS) to study lipid species the murine brain. Using DESI, we detected poly-sialylated gangliosides and their acetylated versions directly from the mouse brain sections as multiply charged ions. IMS simplified imaging data interpretation by separating gangliosides from the other lipid classes into specific trendlines on the mobilograms. Importantly, we detected the gangliosides next to other common lipid species, such as glycerophospholipids, under the same simple experimental conditions. Our findings represent a major improvement in the spatial analysis of fragile lipid species.

We contrasted the DESI-IMS results with matrix-assisted laser desorption ionization (MALDI) coupled to the same ion mobility enabled instrument with an interchangeable source. We further show that complementary lipid information can be obtained, thus allowing to study the murine brain lipidome in a great detail. We briefly discuss the influence of different solvent systems employed for DESI and MALDI. We show that its contribution to the variable extraction and ionization of particular lipid classes is minor, making our DESI and MALDI comparison valid.

In positive mode, DESI-IMS ensured separation of siloxanes carried in the stream of electrospray from the tissue derived molecules in positive mode. We suggest that by imaging potassiated adducts of siloxanes, distribution of potassium across the tissue surface is highlighted. 


\section{Fiducial Markers for Multimodal Mass Spectrometry Imaging}

Based on: Nina Ogrinc Potočnik ${ }^{\wedge}$, Karolina Škrášková ${ }^{\wedge}$, Bryn Flinders, Primož Pelicon, and Ron M. A. Heeren: Gold Sputtered Fiducial Markers for Combined SIMS and MALDI Imaging of Tissue Samples. Anal. Chem. 86, 6781-6785 (2014.) ^Equal contribution 


\title{
4. Fiducial markers for combined secondary ion mass spectrometry and matrix-assisted laser desorption ionization imaging
}

\begin{abstract}
Mass spectrometry imaging (MSI) is a label free technique capable of providing simultaneous identification and localization of biomolecules. A multimodal approach is required that allows for the study of the complexity of biological tissue samples to overcome the limitations of a single MSI technique. Secondary ion mass spectrometry (SIMS) allows for high spatial resolution imaging while matrix-assisted laser desorption ionization (MALDI) offers a significantly wider mass range. Moreover, the two techniques complement each other in the provided molecular information. This, the combination of co-registered SIMS and MALDI images results in detailed and unique biomolecular information. In the following chapter we describe how gold sputtered fiducial markers are created and can be used to ensure a proper overlay and co-registration of the two dimensional images provided by the two MSI modalities.
\end{abstract}

\subsection{Introduction}

Mass spectrometry imaging (MSI) is a label free technique which provides an insight into the elemental and molecular spatial distribution of complex sample surfaces. For this reason it has been applied to a broad range of fields such as biology, biomedicine, pathology, and pharmacology [78, 203]. However, none of the available MSI techniques can individually provide the complete molecular information needed to study complex biological processes. Even though the three commonly used MSI techniques: SIMS, MALDI, and desorption electrospray ionization (DESI) are under constant improvement, each of the techniques provides only limited information about a sample [48, 203]. SIMS for example has the capability of producing highly spatially resolved images of elements and smaller molecules but has a limited mass range. MALDI, on the contrary, provides a complementary molecular information and a broader mass range. It lacks, however, routine high spatial resolution imaging capabilities [204, 205]. The SIMS method is predominantly used for lipid investigation as a result of the substantial fragmentation produced by the primary ion beam [47]. New primary ion sources such as $\mathrm{Ar}_{n}{ }^{+}$and $\left(\mathrm{H}_{2} \mathrm{O}\right)_{n}{ }^{+}$beams $[206,207]$ and use of other cluster ion beams such as $\mathrm{Bi}^{3+}$ and $\mathrm{Au}^{3+}[46,47]$ significantly reduce the molecular fragmentation and extend the application to larger biomolecules. MALDI is typically applied for the detection of larger biomolecules such as peptides and 
proteins, and also offers a complementary perspective for lipid investigation. The combination of the two modalities can in part overcome their individual limitations and provide with a broader insight into the different biomolecular distribution of tissue samples [208-212].

Biological tissue samples are very complex and different tissues are presented to researchers in different forms [62]. Tissue sections without any distinctive anatomical features may cause a problem when trying to combine images acquired by different modalities [204]. Tissue sections may also change their properties during measurements which inhibits correct alignment. In order to properly combine and coregister images from the two above mentioned modalities (MALDI and SIMS) there is a need for specific fiducial markers (FM) [213]. FM have been widely used in MRI [214], PET [215] X-ray and therapeutic proton beam analysis [216] but they are usually inserted directly on the tissue section or inside an organ which changes the tissue integrity. Ink based FM have also been used for co-registration of different imaging modalities and further applied for a $3 \mathrm{D}$ reconstruction of an embedded human breast cancer xenograft [213].

We have adopted a FM technique directly on rat brain and breast cancer (BC) tissue sections to allow for a direct correlation between the high resolution SIMS and MALDI images of the respective samples. The gold sputtered FM described in this paper were clearly observed by both modalities. These new FM are advantageous over other ablation and marker techniques as a result of the known exact dimensions and the well-defined shape of the sputtered FM. They are therefore extremely suitable for subsequent co-registration of individual SIMS and MALDI measurements.

\subsection{Methods}

Sample preparation. Brain tissue of a healthy control rat and $\mathrm{BC}$ tissue of a patient-derived xenograft of triple-negative breast cancer tumor were employed in the experiments. The $12 \mu \mathrm{m}$ tissue sections were prepared with a cryo-microtome (HM525, MICROM, Germany), thaw-mounted on the Indium Tin Oxide (ITO) glass slides (Delta Technologies, USA) and stored in $-80{ }^{\circ} \mathrm{C}$ until further analysis. The sections were placed into a vacuum desiccator to avoid water condensation on the tissue surface prior to the actual MS measurements.

Fiducial markers. The FM were created by sputtering a selected area of $500 \mu \mathrm{m}$ x $500 \mu \mathrm{m}$ on a sample surface with a $22 \mathrm{keV} \mathrm{Au}{ }^{+}$primary ion beam with $2 \mathrm{nA}$ current in direct current (DC) mode for 5 minutes. Test experiments were carried out with blank ITO slides to evaluate the optimal sputtering time and to determine the $\mathrm{m} / \mathrm{z}$ at which the FM were observable. The slides were washed in hexane and ethanol prior to the sputtering process. The FM were sputtered for 1, 3, 5, 10, 15 and 20 minutes. The sputtering time of $5 \mathrm{~min}$ was selected as optimal. In the further experiments with 
the tissue sections the FM were implanted on the glass slide next to the tissue section and on the tissue section itself as shown in Figure 4.1. After the FM implantation the tissue sections were imaged with both techniques over the whole or selected area including the outside FM.

SIMS-MSI. Samples were analyzed using a TRIFT II (Physical Electronics, USA) time of flight secondary ion mass spectrometer (TOF-SIMS) equipped with a

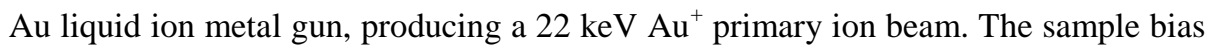
was $3 \mathrm{kV}$. The spectra were calibrated on low mass fragments $\mathrm{H}, \mathrm{CH}_{3}, \mathrm{Na}$ and $\mathrm{Au}$. The images were acquired in positive ion mode with a $128 \mu \mathrm{m}$ raster size per tile resulting in a $500 \mathrm{~nm}$ pixel size or $162.5 \mu \mathrm{m}$ raster size per tile resulting in $635 \mathrm{~nm}$ pixel size for rat brain and $\mathrm{BC}$ tissue, respectively. Acquisition and data analysis were performed by the WinCadence software package (Physical Electronics, USA).
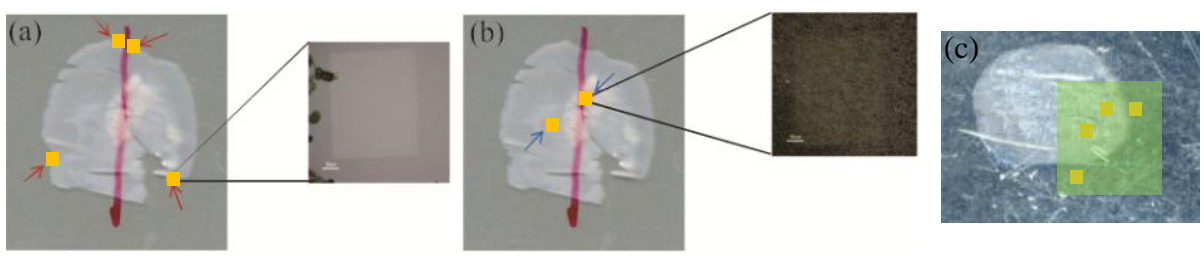

Figure 4.1 Optical images of $12 \mu \mathrm{m}$ thick tissue sections and $500 \mu \mathrm{m} \times 500 \mu \mathrm{m}$ gold sputtered FM indicated by yellow squares. (a) Off-tissue FM adjacent to the brain tissue section (red arrows). (b) On-tissue FM on the brain tissue section (blue arrows). (c) Off- and on-tissue FM on breast cancer tissue section. The large green square indicates the selected SIMS imaged region.

MALDI-MSI. After the SIMS measurement the samples were coated with a matrix solution (10 mg/mL $\alpha$-cyano-4-hydroxycinnamic acid (CHCA) in $70 \%$ methanol solution with $0.2 \%$ TFA) using a vibrational sprayer (ImagePrep, Bruker, Germany). Samples were analyzed on the MALDI-Q-TOF instrument (Synapt HDMS, Waters, UK) in positive ion mode in the mass range $m / z=0-1000 \mathrm{Da}$. Polyethylene glycol (PEG) was used as an external standard for instrument calibration. The images were acquired with a raster step size of 100 and $150 \mu \mathrm{m}$ for brain and $\mathrm{BC}$ tissue, respectively. The data was analyzed using the BioMap software (Novartis, Switzerland). Note that it is imperative to perform the SIMS analysis prior to the MALDI measurements due to the discrepancy in surface damage between the two techniques. MALDI removes more surface material per pixel compared to SIMS that is minimally invasive.

Principal component analysis. Principal component analysis (PCA) was performed on the blank ITO slide datasets to further evaluate the $\mathrm{m} / \mathrm{z}$ characteristic 
for the off-tissue FM. For PCA our in-house developed ChemomeTricks toolbox for MATLAB was used.

\subsection{Results and Discussion}

Blank ITO slide. We performed test measurements of the sputtered FM implanted on a blank ITO slide in order to select the optimal sputtering time and to determine the peaks related to the off-tissue FM. Peaks related to the ITO layer of the glass slides showed correlation with the sputtered areas as shown in Figure 4.2. Peaks at $m / z 115(\mathrm{In}), 229\left(\mathrm{In}_{2}{ }^{+}\right)$, and $246\left(\mathrm{In}_{2} \mathrm{O}^{+}\right)$showed higher intensity within the FM fields. The peak at $m / z 39$ corresponding to potassium was depleted at the FM fields as a result of the DC sputtering procedure. PCA revealed other peaks characteristic for the FM area (Figure 4.3). 5 min sputtering time was selected as optimal when sufficient visibility of the FM at all determined $\mathrm{m} / \mathrm{z}$, was obtained. Longer sputtering periods resulted in distorted FM shapes.

After the deposition of the FM the marked blank ITO slide was covered with matrix and MALDI imaged. Figure 4.2b shows a spectral comparison of a region of interest within 5 min sputtered FM (in red) and within a matrix-only area (in blue). (Note the regions of interest were of the same size.) The peaks related to the FM are marked with arrows and annotated with the respective $m / z$ values. Figure $4.2 \mathrm{c}$ shows images of the selected FM-related peaks. Results of PCA confirmed these $m / z$ being correlated with the FM areas as shown in Figure 4.4. 
a)

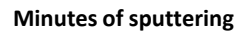

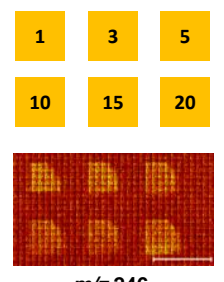

m/z 246

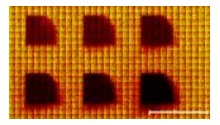

$m / z 39$

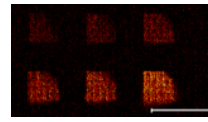

$m / z 318$

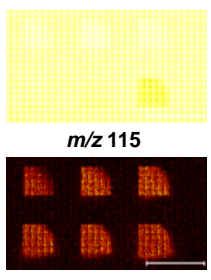

$m / z 360$

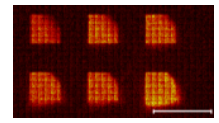

$m / z 229$

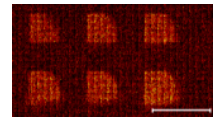

$m / z 376$ b)

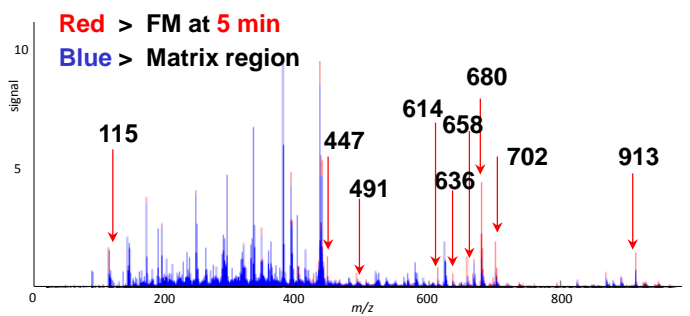

c)

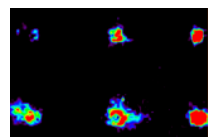

$m / z 115$

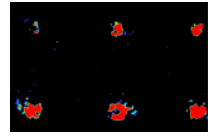

$m / z 680$

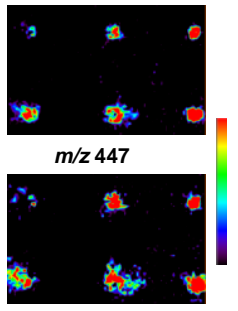

$m / z 913$

Figure 4.2 Blank ITO slide with sputtered FM. (a) High resolution SIMS images of FM at selected $\mathrm{m} / \mathrm{z}$ values with sputtering times of 1, 3, 5, 10, 15 and $20 \mathrm{~min}$. (b) Comparison of MALDI spectra of region of interest within the 5 min sputtered FM and the matrix region. (c) MALDI images of selected $\mathrm{m} / \mathrm{z}$ values of the most abundant FM-related peaks with sputtering times of 1, 3, 5, 10, 15 and $20 \mathrm{~min}$.

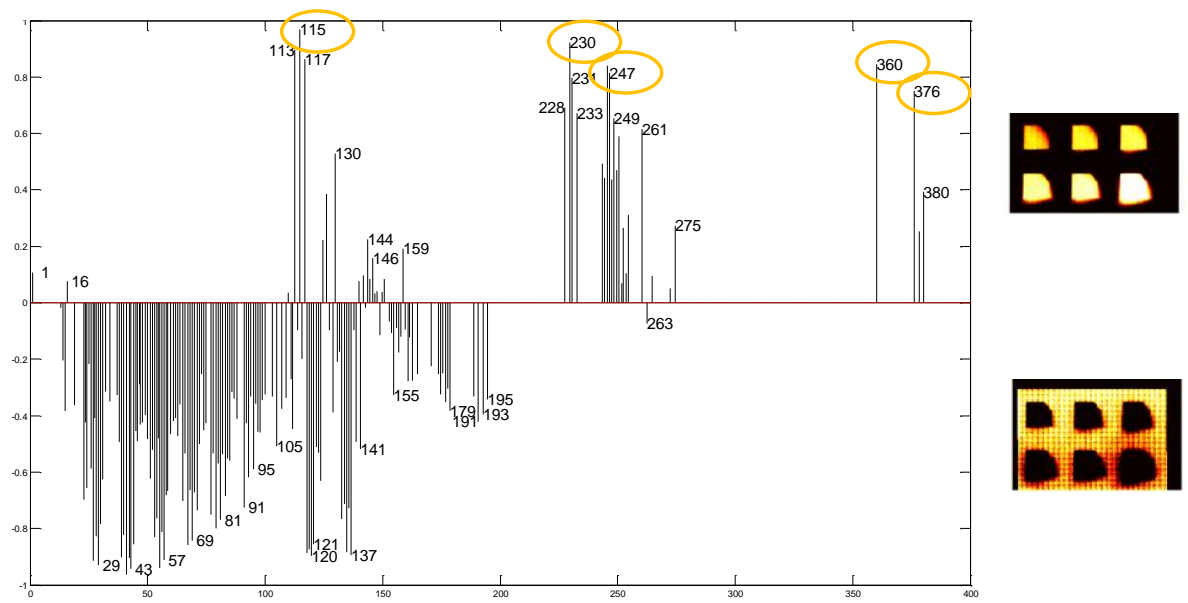

Figure 4.3 Principal component analysis of the blank ITO slide SIMS measurement. Shown are the loadings plot and positive and negative scores images of principal component 1 which clearly separated the fiducial markers area from the background. The positive scores image shows the fiducial markers. The corresponding loadings include the $\mathrm{m} / \mathrm{z}$ typical for a SIMS imaged off-tissue fiducial markers (marked with yellow ovals). 


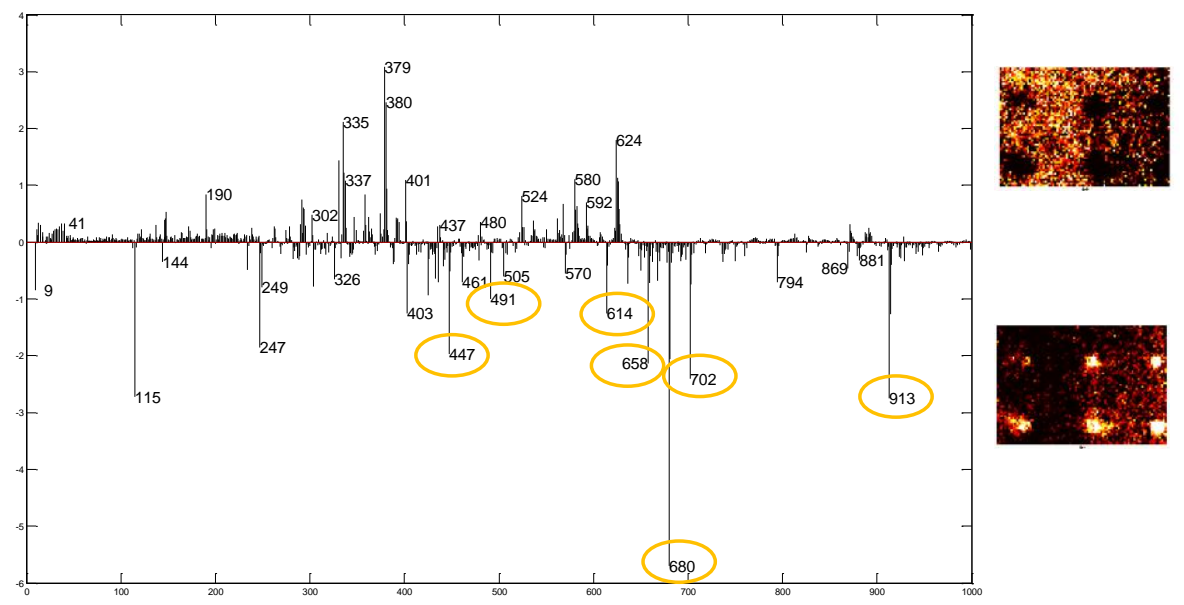

Figure 4.4 Principal component analysis of the blank ITO slide MALDI measurement. Shown are the loadings plot and positive and negative scores images of principal component 4 which clearly separated the fiducial markers area from the background. The negative scores image shows the fiducial markers. The corresponding loadings include the $\mathrm{m} / \mathrm{z}$ typical for a MALDI imaged off-tissue fiducial markers (marked with yellow ovals).

Tissue samples. Off- and on-tissue FM were sputtered next to and on top of the tissue samples as described in Methods. The tissue sections were subsequently imaged using SIMS and MALDI. Figure 4.5 and Figure 4.6 show some of the offand on-tissue FM on brain and BC tissue, respectively. The off-tissue FM are in correlation with the blank ITO slide measurements. Imaged with SIMS they are clearly visible at $m / z, 115\left(\operatorname{In}^{+}\right), 229\left(\operatorname{In}_{2}^{+}\right)$, and $246\left(\mathrm{In}_{2} \mathrm{O}^{+}\right)$as shown in Figure 4.5a-c and Figure 4.6a-c. Measured with MALDI all $\mathrm{m} / \mathrm{z}$ found during the blank ITO slide measurements were also visible. For illustration $m / z, 447$ and 680 are shown in Figure 4.5f-g and Figure 4.6f-g.

The on-tissue FM were observed in the SIMS dataset with a high intensity at $\mathrm{m} / \mathrm{z}$ 39 (Figure 4.5d and Figure 4.6d) and as squares depleted in signal at $\mathrm{m} / \mathrm{z} 184$ (Figure 4.5e and Figure 4.6e). This indicates that the on-tissue FM are potassium rich and phosphocholine (PC) depleted and are likely related to the tissue surface alterations as a result of the DC sputtering procedure. Within the MALDI datasets the on-tissue FM were mostly displayed in MS images of $\mathrm{m} / \mathrm{z}$ higher than $500 \mathrm{Da}$. The individual species likely correspond to various gold clusters. Importantly, FM of the same $m / z$ are observed independent of the tissue type. Images of the selected $\mathrm{m} / \mathrm{z}$ values are shown in Figure 4.5h-i and Figure 4.6h-i. Figure 4.5j and Figure 4.6j show both types of FM in the same image. Such an image can be constructed by selecting broader mass window within the BioMap software. The selected mass window is shown below the images. Spectral comparison of MALDI on- and off-tissue FM is shown in 
Figure 4.7. Table 4.1 summarizes $\mathrm{m} / \mathrm{z}$ typical for off- and on-tissue FM observed by both modalities.

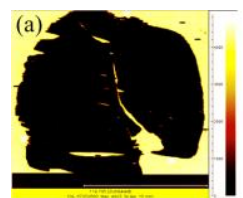

$m / z 115$

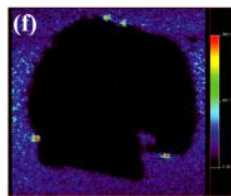

$m / z 447$

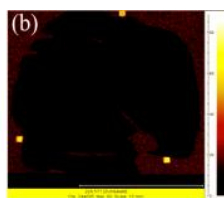

$m / z 229$

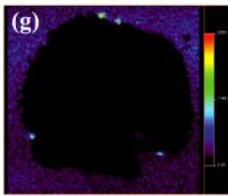

$m / z 680$

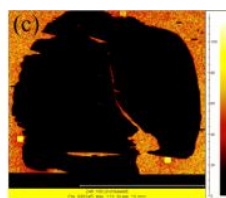

$m / z 246$

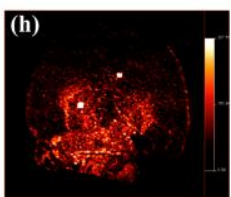

$m / z 807$

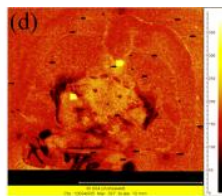

$m / z 39$

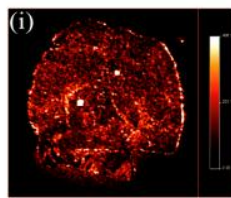

$m / z 849$

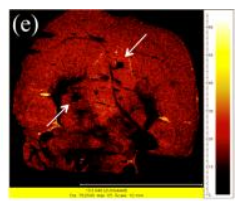

$m / z 184$

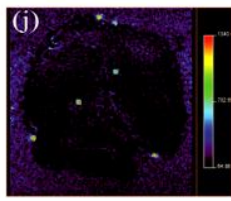

$m / z 868$ - 870

Figure 4.5 Brain tissue section. High spatial resolution SIMS images (a-e). Off-tissue FM: (a) $\mathrm{m} / \mathrm{z} 115$ (indium), (b) $\mathrm{m} / \mathrm{z} 229\left(\mathrm{In}_{2}{ }^{+}\right),(\boldsymbol{c}) \mathrm{m} / \mathrm{z} 246\left(\mathrm{In}_{2} \mathrm{O}^{+}\right.$), on-tissue $\mathrm{FM:} \mathrm{(d)} \mathrm{m} / \mathrm{z} 39$ (potassium) and $(\boldsymbol{e}) \mathrm{m} / \mathrm{z} 184$ (PC head-group) square signal depleted areas are indicated by white arrows. MALDI-MSI images of FM ( $f$-j). Adjacent to the tissue section: $(f) \mathrm{m} / \mathrm{z} 447$ and $(\mathrm{g}) \mathrm{m} / \mathrm{z} 680$, ontissue FM: (i) $\mathrm{m} / \mathrm{z} 807$ and $\mathrm{m} / \mathrm{z}$ 849. Off- and on-tissue FM within the mass window of 868-870 Da.

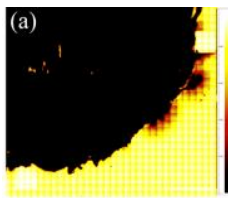

$m / z 115$

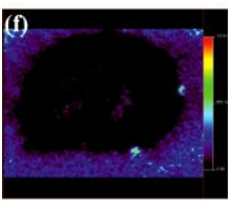

$m / z 447$

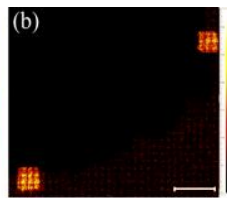

$m / z 229$

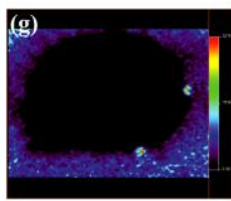

$m / z 680$

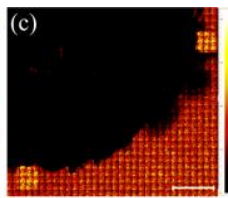

$m / z 246$

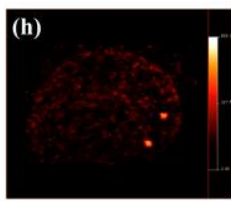

m/z 807

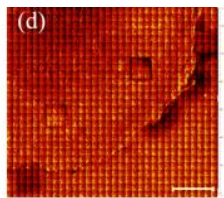

$m / z 39$

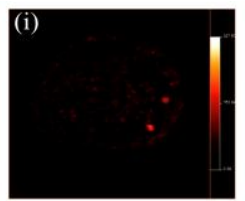

$m / z 849$

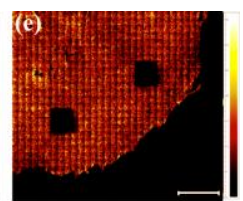

$m / z 184$

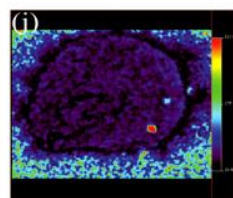

$m / z 868$ - 870

Figure 4.6 Breast cancer tissue. High spatial resolution SIMS images of the selected area (ae). Off-tissue FM: (a) $\mathrm{m} / \mathrm{z} 115$ (indium), (b) $\mathrm{m} / \mathrm{z} 229\left(\mathrm{In}_{2}{ }^{+}\right),(\boldsymbol{c}) \mathrm{m} / \mathrm{z} 246\left(\mathrm{In}_{2} \mathrm{O}^{+}\right.$), on-tissue $\mathrm{FM}$ : (d) $\mathrm{m} / \mathrm{z} 39$ (potassium) and (e) $\mathrm{m} / \mathrm{z} 184$ (PC head-group). MALDI images of FM on the entire tissue section $(f-j)$. Adjacent to the tissue section: $(f) \mathrm{m} / \mathrm{z} 447$ and $(\mathrm{g}) \mathrm{m} / \mathrm{z}$ 680, on-tissue FM: (h) $\mathrm{m} / \mathrm{z}, 807$ and (i) $\mathrm{m} / \mathrm{z}, 849$, (j) off- and on-tissue FM within the mass window of $\mathrm{m} / \mathrm{z}$, 868-870. 


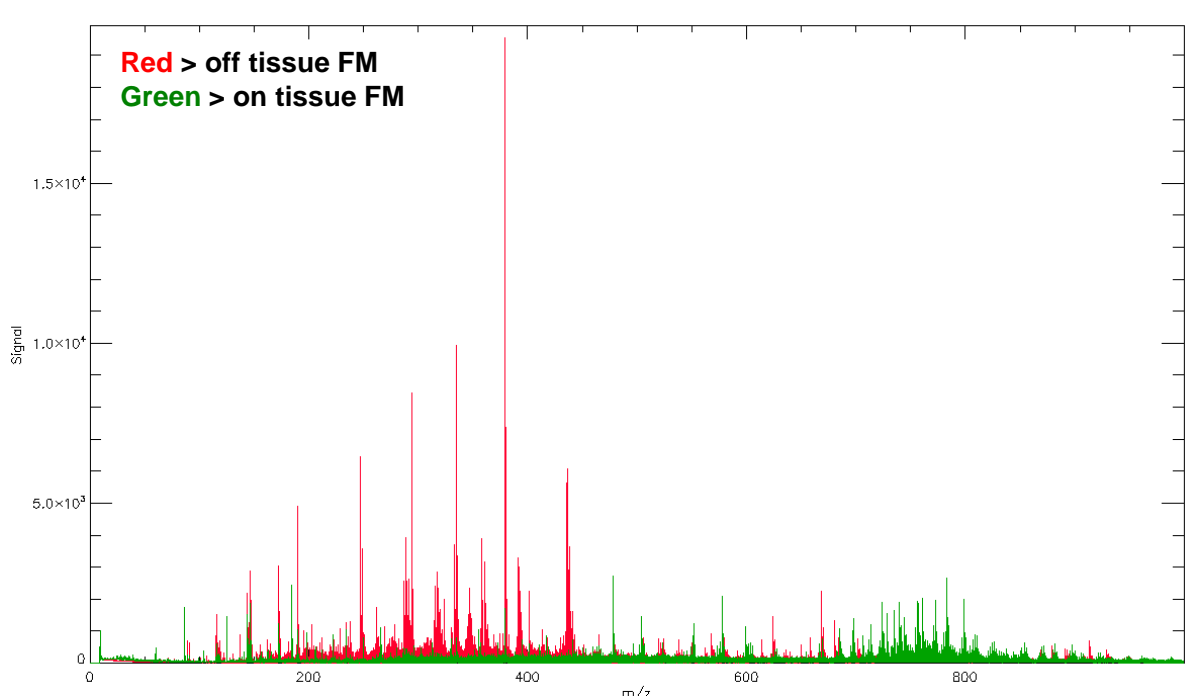

Figure 4.7 MALDI spectra of the off- and on-tissue fiducial markers. Shown are spectra from a region of interest of an off- and on-tissue fiducial marker. The regions of interest were of the same size. The off-tissue FM are observed at lower masses whereas the on-tissue fiducial markers are present mostly in the mass region above 500 Da. The intense red peaks also include matrix peaks, whereas the green peaks include lipid signal coming from the tissue. However, the FM and lipid peaks have slightly different mass defects.

\section{SIMS off-tissue}

$m / z$

115

229

246

360

376

SIMS on-tissue

$m / z$

39

184

(1)

(1)


Co-registration. A demonstration of the easy co-registration using the SIMS generated fiducial markers is provided in Figure 4.8. Figure 4.8a shows a manual multi-modal overlay of a SIMS and a MALDI FM image. The SIMS generated $\mathrm{In}_{2}{ }^{+}$ $(\mathrm{m} / \mathrm{z}, 229)$ and potassium $(\mathrm{m} / \mathrm{z}, 39)$ images from Figure $4.5 \mathrm{~b}$ and $4.5 \mathrm{~d}$ were overlaid in order to display the squares where the FM have been created. The MALDI image of the mass window $\mathrm{m} / \mathrm{z}, 868-879$ from Figure $4.5 \mathrm{j}$ shows the FM with lower spatial resolution. The FM visible by both modalities can be now aligned during the coregistration procedure. Subsequently all MALDI and SIMS images extracted from these two experiments can be overlaid as now all the co-registration coordinates are known. The MALDI FM images are not aligned with all SIMS FM completely due to the nature of matrix crystallization and lower spatial resolution of MALDI measurements. The described workflow can also be applied on selected parts of the tissue samples. This might be especially useful when examining regions of interest with high spatial resolution. For example a co-registration of the selected highresolution SIMS images at $\mathrm{m} / z, 184$ and 229 from Figure 4.6b and 4.6e can be overlaid with the MALDI image of the whole BC tissue section as shown in Figure $4.8 \mathrm{~b}$. The MALDI image for the co-registration consists of an overlay of the distribution of $\mathrm{m} / \mathrm{z}=490$ and the image of the mass window 868-879 from Figure 4.6j with all observed FM.

a)

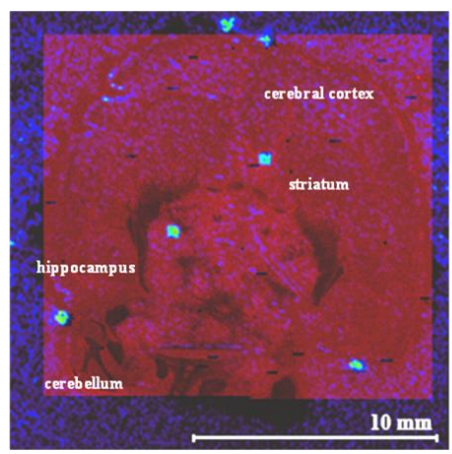

b)

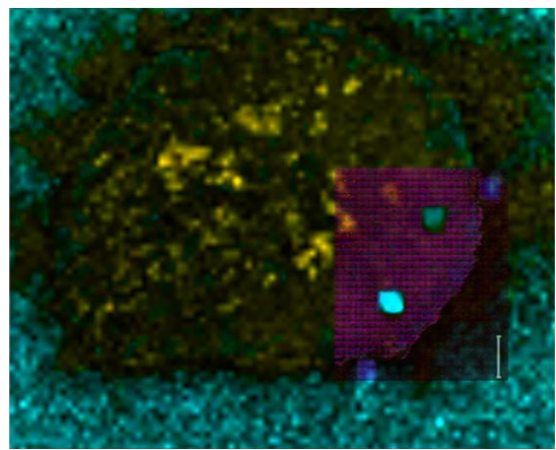

Figure 4.8 Manually co-registered overlay of (a) MALDI image (back) with 6 FM shown within the mass window $\mathrm{m} / \mathrm{z}$ 868-870 in green and the SIMS overlaid images of $\operatorname{In}_{2}{ }^{+}(\mathrm{m} / \mathrm{z} 229)$ and potassium $(\mathrm{m} / \mathrm{z}, 39)$ in red (front). (b) MALDI image of the whole tissue section (back) with 4 FM shown within the mass window $\mathrm{m} / \mathrm{z} 868-870$ and $\mathrm{m} / \mathrm{z} 490$ and the SIMS overlaid image of the selected region of $\operatorname{In}_{2}{ }^{+}(\mathrm{m} / \mathrm{z}, 229)$ and $\mathrm{PC}(\mathrm{m} / \mathrm{z}, 184)$. 


\subsection{Conclusions}

Multimodal imaging requires appropriate co-registration of different images in order to obtain unique complementary information about the structure and composition of tissue sections. This chapter describes a clean and simple way to generate FM both on-tissue and off-tissue by means of a primary ion beam generated by the SIMS instrument. We have shown that the gold sputtered FM can be visualized by SIMS and MALDI imaging on the brain tissue sample as well as on the breast cancer tissue sample. We conclude that the sputter generated FM are suitable technique for MSI image co-registration. 



\section{The Allen Brain Atlas}

Based on: Karolina Škrášková, Artem Khmelinskii, Walid M. Abdelmoula, Stephanie De Munter, Jouke Dijkstra, Myriam Baes, and Ron M. A. Heeren: Precise anatomical localization of accumulated lipids in Mfp2 deficient murine brains through automated registration of SIMS images to the Allen Brain Atlas. J. Amer. Soc. Mass Spectrom. 26, 948-957 (2015). 


\section{Co-registration of secondary ion mass spectrometry images and the Allen Brain Atlas for precise anatomic localization of accumulated lipids}

Mass spectrometry imaging (MSI) is a powerful tool for the molecular characterization of specific tissue regions. Histochemical staining provides anatomical information complementary to MSI data. The combination of both modalities has been proven to be beneficial. However, direct comparison of histology based and MS based molecular images can become problematic due to potential tissue damages or changes caused by different sample preparation. Curated publicly available atlases such as the Allen Brain Atlas (ABA) offer a collection of highly detailed and standardized anatomical information. Direct comparison of MSI brain data to the ABA allows for conclusions to be drawn on precise anatomical localization of the MSI derived molecular signatures. Here we applied secondary ion mass spectrometry imaging (SIMS) at high spatial resolution to brains sections of knock-out mouse models with impaired peroxisomal $\beta$-oxidation. Murine models were lacking MFP2 (D-multifunctional protein) which is involved in degradation of very long chain fatty acids. SIMS imaging revealed deposits of fatty acids within distinct brain regions. Manual comparison of the MSI data with the histological stainings did not allow for an unequivocal anatomical identification of the fatty acids rich region. We further employed an automated pipeline for co-registration of the SIMS data to the ABA. The registration enabled precise anatomic annotation of the brain structures with the revealed lipid deposits.

\subsection{Introduction}

Molecular characterization and classification of specific tissue regions has become crucial in understanding of the mechanisms of various tissue pathologies. In the past decade mass spectrometry imaging has proven to be a tremendously useful technique for the spatial investigation of molecular tissue patterns [34, 64, 78, 217 , 218]. The uniqueness and strength of MSI lie in the ability to simultaneously localize and identify hundreds of molecules from a tissue surface in a single experiment. MSI is also referred to as molecular histology [11, 64]. It can tell apart distinct tissue regions even if they are not differentiated by any of the established histochemical methods. MSI allows for partitioning of the tissue surface into regions with the same or similar MS profiles. Hence, it enables the investigation of molecular changes that 
occur prior to or without any morphological changes [219] and the identification of hidden anatomical features.

The better the spatial resolution of a MSI experiment, the finer structures of the tissue sample can be revealed. Currently the best spatial resolution within the MSI field is provided by secondary ion mass spectrometry instruments. The spatial resolution achievable with SIMS reaches well below $1 \mu \mathrm{m}$ [218]. SIMS makes use of a primary ion beam to probe the sample surface. The mass range of the examined molecules is limited because the impact of the atomic ion beam with the substrate causes extensive molecular fragmentation. Modern molecular ion or cluster ion beams limit the extent of fragmentation. SIMS has been used for the investigation of lipids and inorganic fragments in the study of several biological samples [64, 218].

Employment of multivariate statistical analysis to MSI datasets helps to reveal / highlight MS-profiled tissue regions. Principal component analysis and clustering methods are frequently used in probing the enormous MSI dataload [217, 220]. Results of such a computational segmentation are commonly compared with the results obtained by (immuno)histochemical staining of the same or an adjacent tissue section. The complementary information provided by MSI and histology can then be advantageously combined. MS acquired and the histology-based optical images are typically co-registered for an accurate and efficient comparison. So far this has mostly been done manually with the guidance of fiducial markers or using clear anatomical detail [213, 221]. Matusch et al. took advantage of a commercially available software (Pmod) for alignment of histology and MS images [222]. The general unavailability of the programme, however, prevents from the common use in academia. Recently progress has been made by automating the histology-MSI coregistration process. Abdelmoula et al. [223] described methodology based on tdistributed stochastic neighbour embedding (tSNE) technique. tSNE is a non-linear dimensionality reduction technique developed by van der Maaten et al. [224]. It highlights specific anatomical regions representing the global MSI dataset. The regions can be subsequently used as markers for the alignment. It was demonstrated [223] that tSNE representation of MSI data reveals enough anatomical details that can be treated as landmarks for its registration to histology.

Various folds, tears, or general surface changes of the tissue sections, resulting from sample preparation or from MSI measurements, can significantly hamper their histological evaluation. This phenomenon is accentuated when dealing with fine structural details. It becomes thus plausible to compare MSI datasets to curated anatomical atlases. These contain highly standardized data that describe the anatomy of particular tissue types in high detail. Rodent brain remains one of the most frequently employed tissue types analyzed with MSI. Rodents are often used as models of various neurological and neurodegenerative diseases [64] and also serve as a model organism for mammalian brain development studies and behavioural 
genetics [225]. Example of such a curated atlas commonly employed in brain research is the Allen Brain Atlas (ABA) (http://www.brain-map.org/) [225]. ABA is a freely accessible database of gene expression and neuroanatomical reference data in mouse brain, developing mouse brain and human brain [221]. ABA provides standardized high-resolution histological images combined with hierarchically organized taxonomy of the respective brain regions. Combination of the histological and anatomical information extracted from the ABA with the molecular information provided by MSI can become a powerful tool for brain tissue analysis, and in chemical neuroscience. A couple of papers recently published describe pipelines for alignment of MSI and the ABA data [221, 226]. The methodologies are based on the use of a histochemically stained tissue section that was previously MS imaged. The stained sample serves as an intermediate between the ABA and MSI data. Once MS and $\mathrm{ABA}$ images are aligned, direct conclusions about anatomical localization of MS signal and the affected genetic pathways can be made.

In our inhere presented experiments, $M f p 2$ (multifunctional protein 2) deficient mouse models were involved. MFP2 is a protein that is responsible for formation of bile acids and the degradation of pristanic acid and the very-long chain fatty acids (VLCFA) [227]. $M f p 2^{-1-}$ mice suffer from severe neuromotor dysfunctions and die before the age of 6 months [228]. Histologically, severe neuroinflammation and cerebellar degeneration are observed. Histochemistry showed presence of lipid droplets especially within ependymal cells along the entire ventricular system and in the molecular layer of the cerebellum [229]. Here we imaged $M f p 2^{-/}$brain sections of three biological replicates at high spatial resolution using SIMS. We revealed accumulation of fatty acids (FA) in a sharply defined region underneath the cerebellum. Since manual comparison of the SIMS data with the histological staining did not allow for an unequivocal identification of the brain region with the lipid deposits, the MS images were further co-registered to the ABA. The fully automated pipeline of the co-registration enabled precise anatomic annotation of the FA rich hotspots.

\subsection{Methods}

Mouse breeding. The generation and breeding of $M f p 2^{-/}$mice has been in detail described elsewhere [228]. Mice were bred in the specific-pathogen free animal housing facility of KU Leuven, had ad libitum access to water and standard rodent food, and were kept on a 12 hour light and dark cycle. All animal experiments were performed in accordance with the "Guidelines for Care and Use of Experimental Animals" and fully approved by the Research Ethical Committee of the KU Leuven (177/2012). 
Tissue preparation. 12 -week old $M f p 2^{-/}$mice and a wild type (WT) littermate were euthanized by an intraperitoneal Nembutal injection. Mice were perfused with cold ammonium acetate $(50 \mathrm{mM}, \mathrm{pH} 7.3)$ in a $4{ }^{\circ} \mathrm{C}$ room to prevent lipid degradation. Brains were rapidly removed, cut in the midsagittal plane and frozen on dry ice. Brains were kept in glass vials at $-80^{\circ} \mathrm{C}$ before further processing. Contamination and lipid diffusion were avoided by washing the working space and instruments with hexane.

Tissue preparation for SIMS-MSI. $12 \mu \mathrm{m}$ thick tissue sections were obtained using cryomicrotome (Microm International, Germany). The sections were thaw mounted [230] onto Indium Tin Oxide (ITO) covered glass slides (Delta Technologies, USA). The tissue sections were stored at $-80{ }^{\circ} \mathrm{C}$ until further analysis. Prior to MSI the tissue sections were placed into a vacuum desiccator for 20 minutes to reach room temperature while avoiding condensation of water on top of their surface. The tissue sections were covered with a $1 \mathrm{~nm}$ gold layer using a Quorum Technologies SC76440 sputter coater (New Haven, UK). Gold coating enhances the molecular signal in Metal-Assisted SIMS through increases stopping power of the primary ions and an improvement of the surface conductivity.

SIMS Mass Spectrometry Imaging. The samples were analyzed on TRIFT II (Physical Electronics, USA) time of flight secondary ion mass spectrometer (TOFSIMS) equipped with a Au liquid ion metal gun, producing a $22 \mathrm{keV} \mathrm{Au}{ }^{+}$primary ion beam. A region of interest (roughly $6 \times 6 \mathrm{~mm}$ ) that included the cerebellum and brain stem was imaged in a mosaic mode consisting of $64 \times 64$ squared tiles. Each tile contained $256 \times 256$ pixels. The size of a tile was $98.2 \mu \mathrm{m}, 106.3 \mu \mathrm{m}$, and $82.8 \mu \mathrm{m}$, for the three knock-out replicates (KO1, KO2, KO3), respectively. The size of a tile for the WT mouse was $78.1 \mu \mathrm{m}$. The spatial resolution of the raw data was well below $0.5 \mu \mathrm{m}$. Acquisition time per tile was set to $11.4 \mathrm{~s}$. Spectra were acquired in negative mode within the mass range of 1-1,000 Da. Spectra were first calibrated on low mass fragments $(\mathrm{O}, \mathrm{Cl}, \mathrm{Au})$, and then re-calibrated on gold clusters.

Data pre-processing. The data was converted into MATLAB format employing our in-house developed ChemomeTricks toolbox for MATLAB (MathWorks, Natick, MA). Mass channels were binned into 0.05 Da wide mass bins. An average spectrum of all pixels was used for subsequent peak picking. Peak picking was performed on a basepeak mass spectrum with an algorithm which is in detail described elsewhere [231]. The peak list contained 879, 1065, and 1443 peaks for KO1, 2 and 3, respectively, and 1400 peaks for the WT. The peak lists were used to integrate each pixel's mass spectrum within the respective raw datasets. During the integration the pixels were spatially binned resulting in 256x256 pixels datasets. A multiorder correction algorithm based on linear discriminant analysis was applied to remove image distortions caused by the mosaic character of the data acquisition. The algorithm is in detail described elsewhere [232]. The number of iterations differs 
according to the data quality. The number of iterations for presented datasets was 3 , 7, and 8 for the three KO replicates, respectively, and 5 for the WT. Finally the SIMS data was recalibrated on gold coating related peaks with well known $m / z$ values in MATLAB environment using polynomial fit.

Principal component analysis and Hierarchical cluster analysis. The preprocessed data was further used for PCA and hierarchical cluster analysis (HCA) which were both performed using the ChemomeTricks toolbox. The data was normalized on TIC and autoscaled prior to PCA. (N.B. By autoscaling we refer to a process in which each column of the data matrix was divided by its standard deviation and its mean was subtracted.) The first principal component separating the tissue from the surrounding was used to remove pixels of the latter. HCA was performed with the first 20 PCs employing the correlation distance and the average linkage approach.

Tissue staining. After SIMS imaging the tissue was stained with Cresyl violet (Nissl stain) and scanned using a Mirax DESK digital slide scanner (Zeiss, Germany).

MSI data registration to the Allen Brain Atlas. The pipeline used to automatically register the SIMS datasets to the ABA is based on a combination of two recently published techniques developed by Abdelmoula et al.: 1.) automatic registration of MSI data to histology using tSNE [223] and 2.) the automatic registration of histology to the ABA [221]. The whole co-registration pipeline can be divided into 5 main steps: i.) Pre-processing of the histological images, ii.) Registration of the pre-processed ABA histology to the pre-processed experimental histology, iii.) tSNE of the SIMS dataset, iv.) Registration of the tSNE representation of the SIMS dataset to the experimental pre-processed histology, and v.) Final coregistration of the SIMS dataset and the ABA. A diagram of the complete coregistration workflow together with a more detailed description of all five steps are included in Figure 5.1. For more details the readers are kindly referred to the corresponding publications. 


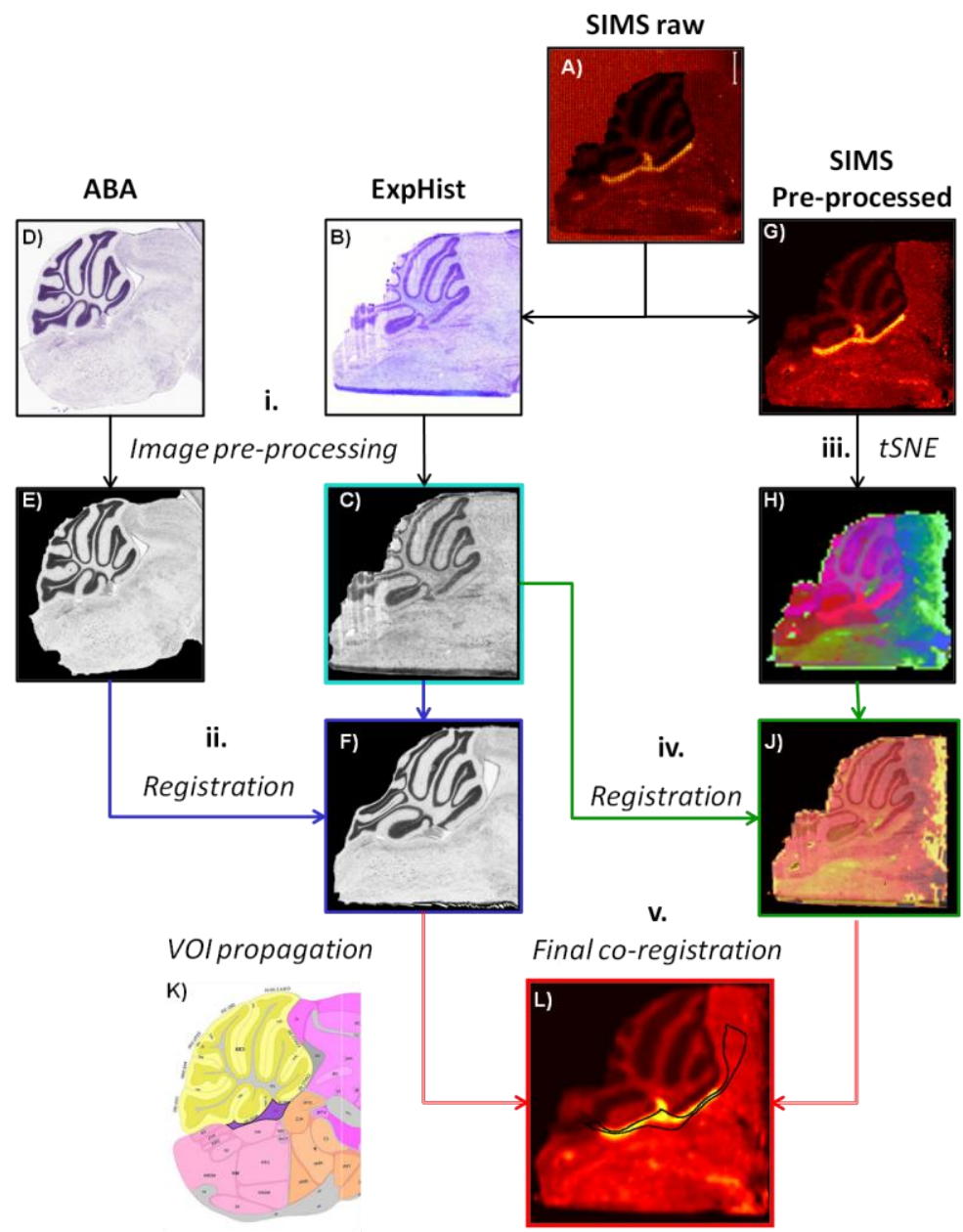

Figure 5.1 Diagram and description of the complete co-registration workflow. The preprocessed high resolution experimental histology (panel $C$ ) plays a pivotal role in the coregistration pipeline. It serves as an intermediate between mass spectrometry imaging (MSI) data and the Allen Brain Atlas (ABA). The whole co-registration pipeline can be divided into 5 main steps:

i.) Pre-processing of the histological images: Both the experimental histology (Figure 5.1B) and the $A B A$ reference section from the experiment-matching brain depth (Figure 5.1D) were pre-processed to exclude background noise, correct for potential image acquisition artefacts, and to maximize the contrast. The images were classified into two clusters using $k$-means clustering, followed by morphological operations to close any potential gaps in the clustered images. The resulting binary masks were used to separate the tissue from the background.

ii.) Registration of the pre-processed ABA histology (Figure 5.1E) to the pre-processed experimental histology (Figure 5.1C): Registration was performed in a coarse-to-fine process. First, an affine registration was performed for rough global alignment and scaling. 
Subsequently, a multi-resolution non-rigid B-spline registration was applied. A Gaussian image pyramid was employed in all registration steps applying three resolutions for the affine, and twelve for the B-spline part. Mutual information was used as a similarity metric. The registration was implemented using the open source image registration toolbox Elastix. [217] For more details see reference. [221]

iii.) tSNE of the SIMS dataset: tSNE was applied on the pre-processed SIMS dataset (Figure $5.1 G)$ to find its low-dimensionality representation. It was performed using the default settings described in [224] and the tSNE Matlab toolbox. [233]

iv.) Registration of the tSNE representation of the SIMS dataset (Figure 5.1H) to the experimental pre-processed histology (Figure 5.1C): The tSNE image was registered to the experimental histological image and the obtained deformation field (Figure 5.1J) was used to deform each mass channel to the histological reference space. For more details see reference. [223]

v.) Final co-registration of the SIMS dataset and the ABA: Using the information provided by the deformation fields obtained in steps ii.) and iv) the selected ABA anatomical labels (volumes of interest - VOIs, Figure 5.1K), were finally propagated onto each mass channel of interest (Figure 5.1L). The VOIs are hierarchically organized, and sorted through the ABA anatomical segmentation maps.

\subsection{Results}

SIMS images tissue samples at the highest spatial resolution of current MSI techniques. Owing to the character of its probing beam, the spatial resolution of SIMS experiments can reach below $1 \mu \mathrm{m}$. Here we employed SIMS imaging to sagittal brain sections of $M f p 2$ deficient mouse models. The MSI experiments were designed so that the cerebellum and brain stem were imaged at high spatial resolution. The preselection of the region of interest (ROI) was guided by pathological features such as cerebellar degeneration and neuroinflammation in the brainstem of $\mathrm{Mfp}^{-/}$mice [229]. In addition, Oil Red O positive lipid deposits have been identified in the ependymal cells of the entire ventricular system and in cerebellar Bergmann glia fibers [229].

In the raw SIMS images of KO1 some lipid-related mass channels \{e.g. oleic acid (18:1) at $\mathrm{m} / \mathrm{z} 281\}$ showed a significant spatial pattern manifested as a sharply denoted region beneath the cerebellum (Figure 5.2a). The pre-processing of the raw data significantly improved the image quality even though the spatial resolution was compromised due to the employed spatial binning (Figure 5.2b). PCA further highlighted the region which is clearly visible on the negative score image of the third principal component (PC) (Figure 5.2c). (N.B. The negative score image is an image of pixels with negative score values.) The negative part of the corresponding loadings plot shows amongst others mass channels belonging to various fatty acids (FA) marked with blue arrows in Figure 5.2d. Most of them represent fatty acids with chain lengths ranging from 16 to 24 carbons. PCA enabled molecular characterization 
of the revealed hotspot through association of a specific molecular pattern. All three biological replicates manifested the presence of the fatty acids-related hotspot. The results of PCA for the two KO biological replicates are presented in Figure 5.3. The WT littermate did not manifest the region-specific accumulation of fatty acids. Figure 5.4 shows results of HCA of WT and KO1 mouse. Shown are RGB overlays of three clusters: green and blue clusters contain mass channels of FA. Whereas a specific spatial pattern formed by the FA mass channels is formed in the KO models, no such a FA rich hotspot was observed in the WT mouse.
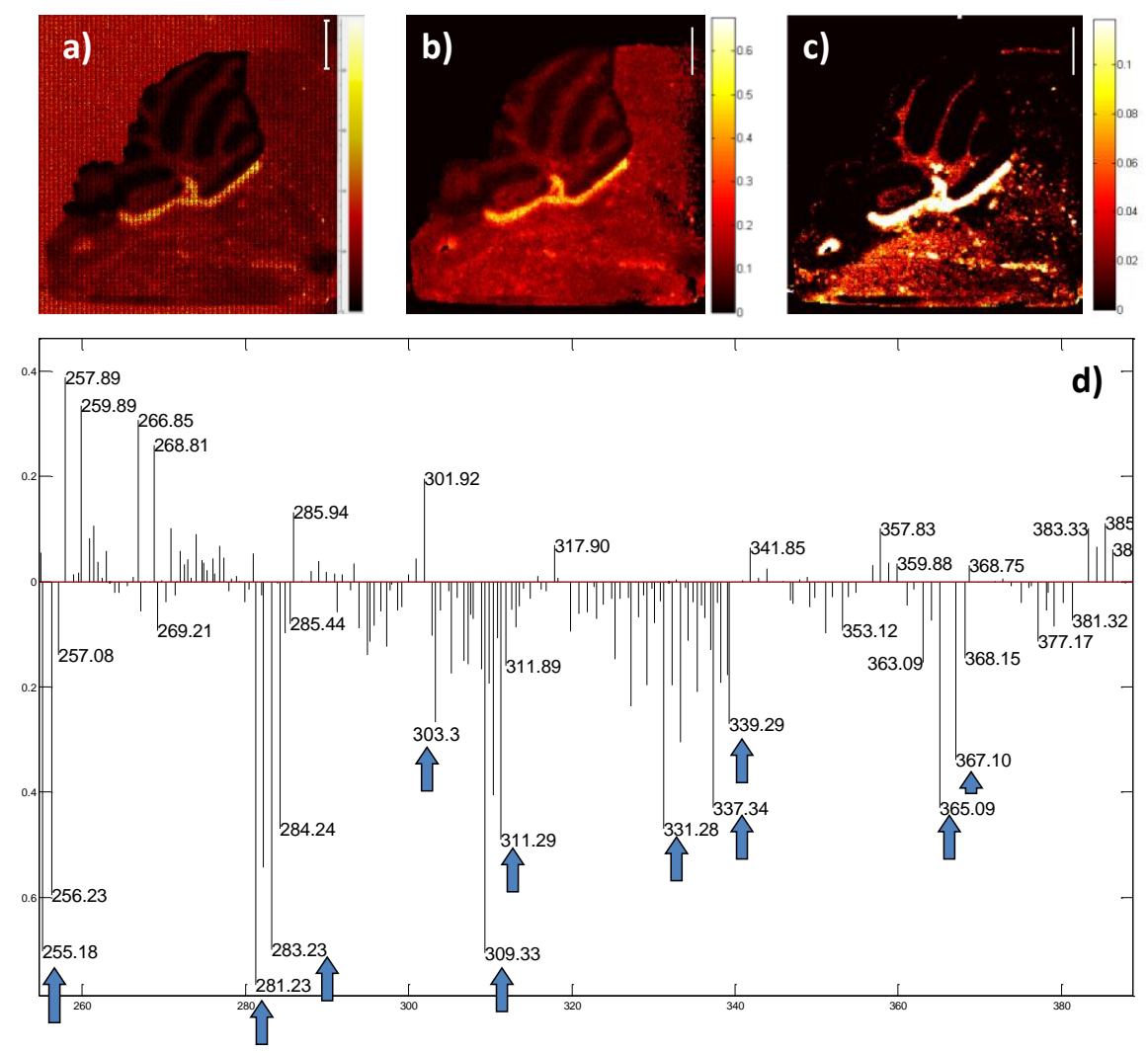

Figure 5.2 a) Raw data MS image of $\mathrm{m} / \mathrm{z}, 281$ (oleic acid 18:1), b) MS image of $\mathrm{m} / \mathrm{z}, 281$ after data pre-processing, c) Score image of pixels with negative score values of principal component $(P C)$ number $3, \boldsymbol{d})$ Loadings plot corresponding to PC 3. The mass channels in the negative part of the loadings plot are associated with the hotspot highlighted on the score image (c). The mass channels marked with the blue arrows correspond to the following fatty acids (FA): m/z 255 FA (16:0), m/z 281FA (18:1), m/z 283 FA (18:0), m/z 303 FA (20:4), m/z 309 FA (20:1), m/z 311 FA (20:0), m/z 331 FA (22:4), m/z 337 FA (22:1), m/z 339 FA (22:0), $\mathrm{m} / z, 365$ FA (24:1), m/z 367 FA (24:0). Scale bar $1 \mathrm{~mm}$. 
a)

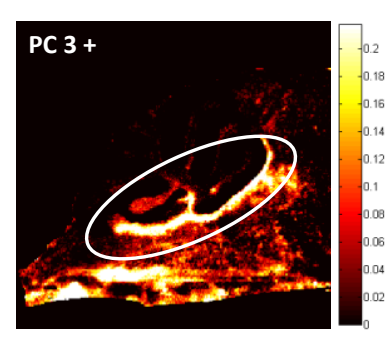

c)

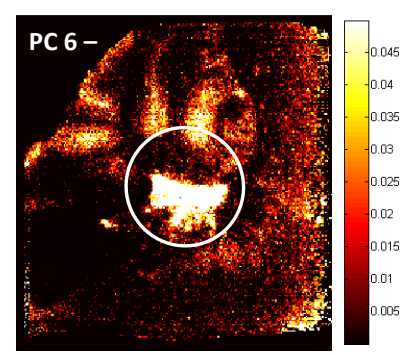

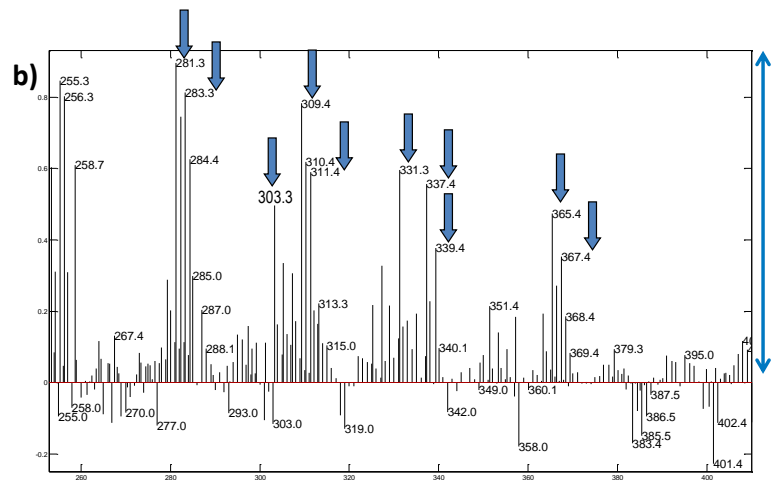

d)

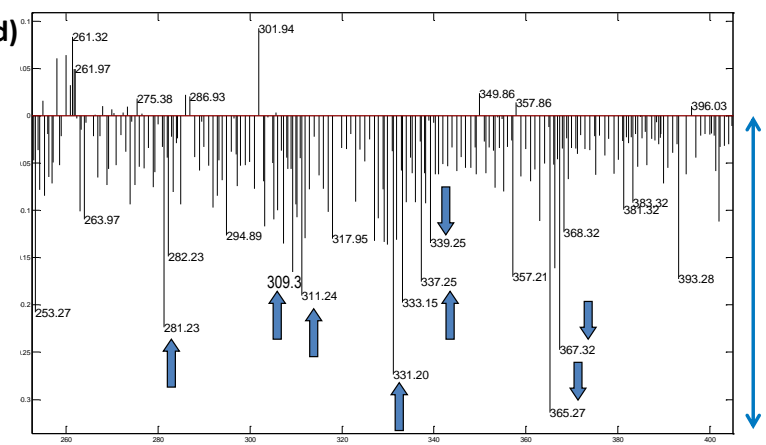

Figure 5.3 Validation of results of the principal component analysis for two biological replicates. Shown are score images of principal components that highlighted the tissue region localized underneath the cerebellum, KO2 in panel a), and KO3 in panel c). The ranking of the principal components (PCs) showing the hotspot differed. Whereas for KO1 and KO2 the hotspot was visible on PCs 3, KO3 highlighted the hotspot through PC 6. Panels b), and d) show the corresponding loadings plots with the fatty acids marked via blue arrows. 

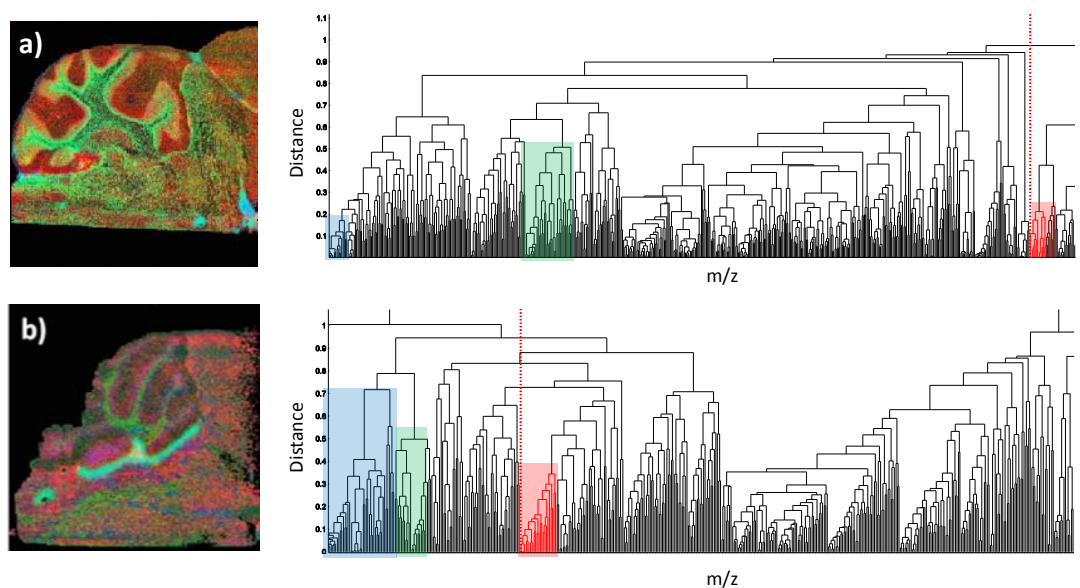

Figure 5.4 Comparison of hierarchical cluster analysis of a) wild type and $\boldsymbol{b}$ ) knock-out mouse KO1. Green and blue clusters represent fatty acids containing clusters. Red clusters contain chlorine. Whereas FA are present in the WT mouse, no accumulative pattern is observed.

After SIMS imaging the tissue sections were stained using Cresyl violet and optical images were recorded. Figure 5.5a and Figure 5.5b show the ABA and the experimental histology for KO1, respectively. Based on their manual comparison and taking into account the shape of the observed FA hotspot, it was tentatively linked with the $4^{\text {th }}$ ventricle and partly with the cerebellar aqueduct. In order to assess an unequivocal anatomical localization of the hotspot, the respective KO SIMS datasets were further coregistered to the corresponding ABA reference sections. Figure 5.5c-d shows the pre-processed histologies of $\mathrm{KO} 1$ and their co-registration result, e. In black contour are shown the volumes of interest (VOIs), i.e. corresponding $\mathrm{ABA}$ labels, of the $4^{\text {th }}$ ventricle and the cerebellar aqueduct. 

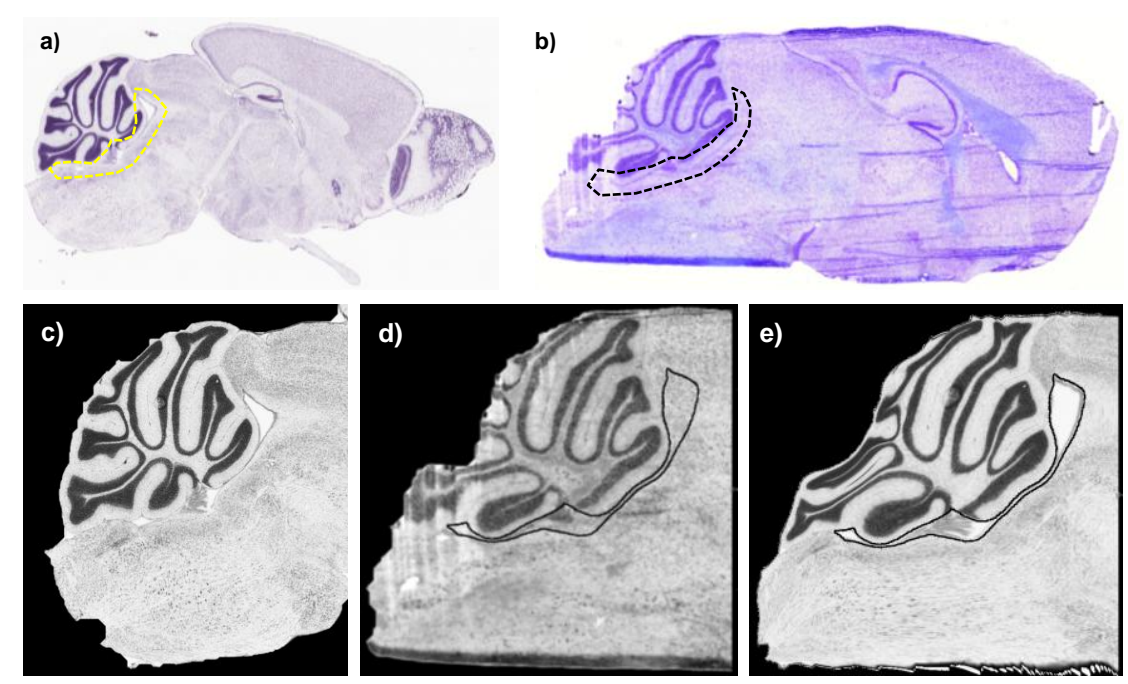

Figure 5.5 Comparison of the raw and pre-processed histologies. a) the raw ABA reference section for the $\mathrm{KOl}, \boldsymbol{b})$ the raw $\mathrm{KOl}$ experimental section, $\boldsymbol{c})$ the pre-processed experimental histology, $\boldsymbol{d})$ the pre-processed ABA histology, and $\boldsymbol{e})$ the resulting image after registering the image in c) to the image in d). The black and yellow contour in panels a) and b) manually delineate the $4^{\text {th }}$ ventricle and the cerebellar aqueduct. Contours in panels d) and e) represents the ABA VOIs of those particular structures.

Figure 5.6 shows the final MSI-ABA co-registration results for the three biological replicates. The analyzed tissue sections of the respective knock-outs were taken from different brain depths: The KO1 was cut approximately $700 \mu \mathrm{m}$ deep away from the brain mid-line, the $\mathrm{KO} 2$ was sectioned right at the brain midline, and the KO3 section was acquired from a site more than $1 \mathrm{~mm}$ far from the mid-line. The cross-section of particular brain structures naturally changes within the brain depth. Also the FA rich region observed in the all three KO replicates was manifested in different shapes. The ABA reference sections were selected as follows: KO1 - No.18, $\mathrm{KO} 2$ - No.21, and KO3 - No.16, based on their spacing in the ABA. The correct selection of the corresponding $\mathrm{ABA}$ reference section is essential as is further explained in the "Discussion" section. Figure 5.6a, e, j show the anatomical segmentation maps with the VOIs of the selected ABA reference sections for the respective samples. The VOI of the $4^{\text {th }}$ ventricle is highlighted in violet colour. The shape of the fatty acids-related hotspot for the KO1-3 is in high contrast shown in panels $b, f$, and $k$ depicting the score images resulting from the PCA of the respective SIMS datasets. Panels c, g, l, and d, h, m show the final results of the SIMS-ABA coregistration. The VOI of the $4^{\text {th }}$ ventricle ( 44 r lateral recess) is propagated onto the mass channels $339\{(\mathrm{FA}(22: 0)\}$, and $365\{(\mathrm{FA}(24: 1)\}$, respectively. The anatomical label is visible as black (or white where the contrast of the image required) contour. 
Note that for $\mathrm{KO} 1$ and $\mathrm{KO} 2$ a VOI of the cerebellar aqueduct $(A Q)$ was also projected onto the MS images. The label(s) and the hotspot co-localize thus confirming the presence of fatty acids in the $4^{\text {th }}$ ventricle. Note that the VOIs extracted from the ABA and the FA hotspots demonstrate almost identical shapes, even though in some cases the hotspot signal does not fill the label to the full extent. Hence making the link between the hotspot and the anatomical label stronger. The match is valid for all three KO replicates although the shape of the hotspot varies due to the different sectioning depths. Figure 5.7 shows an overview of all FA mass channels labelled with the $V 4$ r lateral recess and $A Q$ VOI.
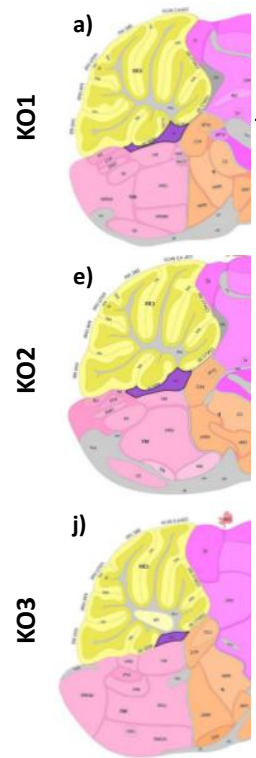

ABA
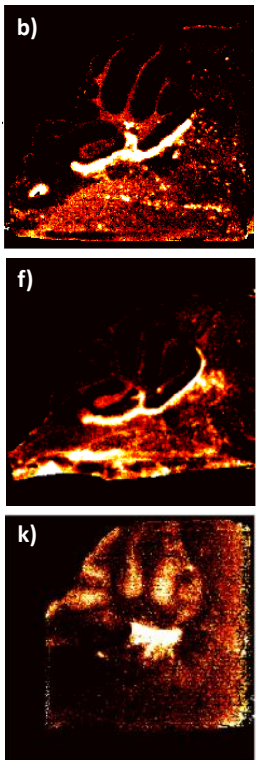

PCA

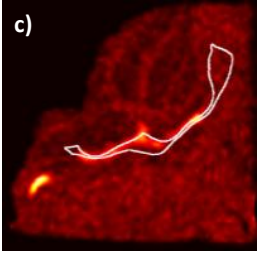

g)
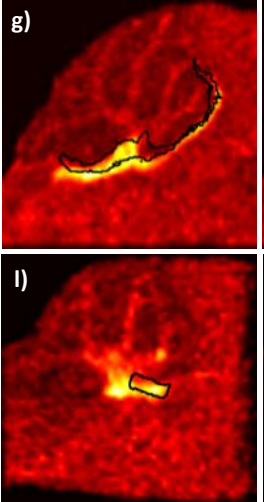

$m / z 339$
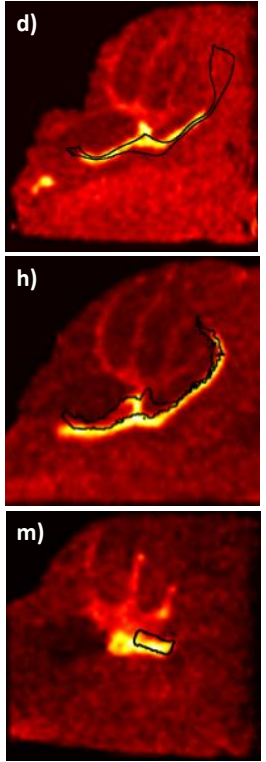

$m / z 365$

Figure 5.6 Results of the final ABA-MSI co-registration presented for three knock-out biological replicates $(\mathrm{KO}, 2,3)$. Panels $\boldsymbol{a}), \boldsymbol{e})$, and $\boldsymbol{j})$ show the anatomical segmentation map of the ABA reference section used for the co-registration pipeline of the respective samples. Highlighted in violet colour is the region of the $4^{\text {th }}$ ventricle. Panels $\left.\left.\boldsymbol{b}\right), \boldsymbol{f}\right)$, and $\boldsymbol{k}$ ) show corresponding score images of the principal components that extracted the hotspots correlated with the presence of fatty acids in the three knock-outs. Panels $\boldsymbol{c}$ ), $\boldsymbol{g}$ ), $\boldsymbol{l}$ ) and $\boldsymbol{d}), \boldsymbol{h}), \boldsymbol{m})$, depict co-registration examples of two mass channels correlated with the hotspots ( $\mathrm{m} / \mathrm{z} 339$ - fatty acid (22:0), and $\mathrm{m} / \mathrm{z} 365$ - fatty acid (24:1), respectively). In black (or white) is shown the label of the $4^{\text {th }}$ ventricle (and for KO1 and KO2 also of cerebellar aqueduct). The scalebar indicates a $1 \mathrm{~mm}$ distance. 

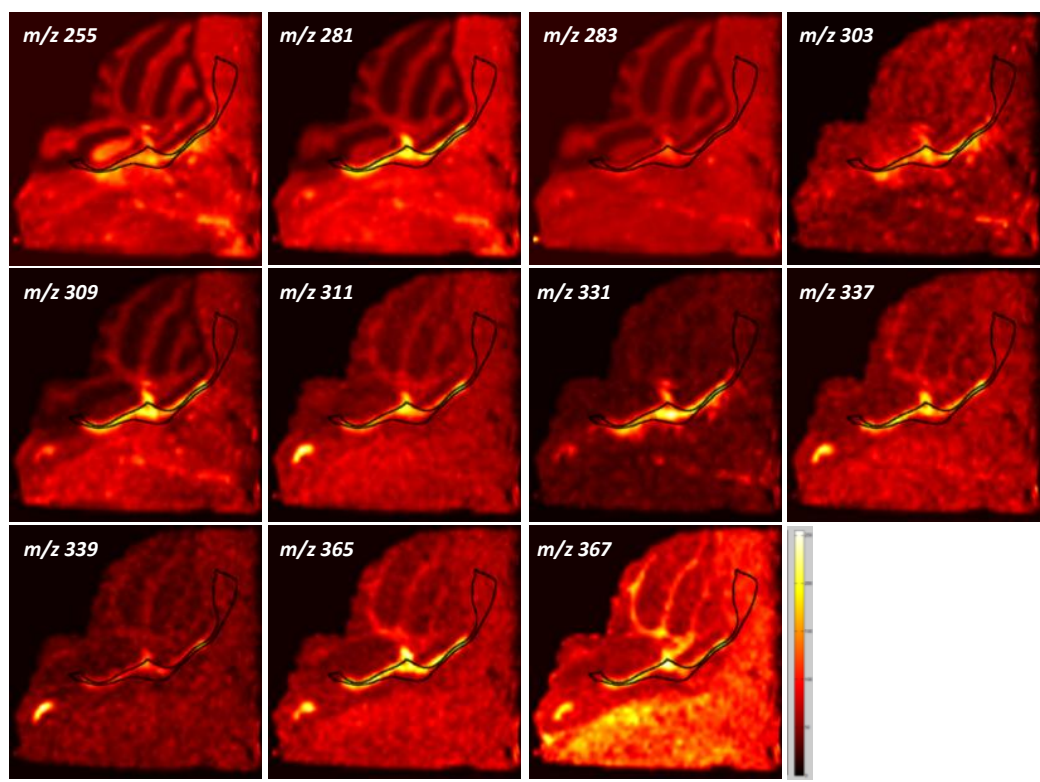

Figure 5.7. Overview off all fatty acids mass channels for KO1 co-registered to the ABA. Shown are mass channels which were correlated with the hotspot via the principal component analysis. Highlighted in white black is the anatomical region of the 4th ventricle and the cerebellar aqueduct. The mass channels correspond to the following fatty acids (FA): $\mathrm{m} / \mathrm{z} 255$ FA (16:0), m/z 281FA (18:1), m/z 283 FA (18:0), m/z 303 FA (20:4), m/z 309 FA (20:1), m/z 311 FA (20:0), m/z 331 FA (22:4), m/z 337 FA (22:1), m/z 339 FA (22:0), m/z 365 FA (24:1), $m / 2367$ FA (24:0).

\subsection{Discussion}

MSI has the ability to extract anatomical features from tissue sections based on their molecular profiles. MSI distinguishes tissue regions even if they cannot be differentiated by conventional histochemical approaches. The combination of both, however, is beneficial as they provide complimentary information. Histochemical information, for instance, can be used to assign anatomical labels to the regions highlighted by MSI. Even though manual inspection is possible and represents an easy and fast way of MSI-anatomy comparison, it does not provide sufficient precision. Figure 5.8 shows the results of our ABA-MSI automated co-registration for KO3. A manual comparison of the ABA anatomical segmentation maps with the MSI data would assign different anatomical labels to the observed hotspot, namely: the $4^{\text {th }}$ ventricle ( 44 r lateral recess) (Figure 5.8a), medial vestibular nucleus $(M V)$ (Figure 5.8b), and laterodorsal tegmental nucleus (LDT) (Figure 5.8c) (N.B. The structures are highlighted in violet colour in the respective anatomical maps.) The MSI-ABA co-registration allowed for correct anatomical identification. To 
demonstrate this, the listed labels were propagated onto the mass channel 339 \{(FA $(22: 0)\}$. From panels d-e it is visible that the VOI V4r lateral recess matches the fatty acids hotspot. Even though the MSI signal does not fill in the whole VOI, the shapes of the hotspot, and the deformed $\mathrm{V4r}$ lateral recess label are close to identical.
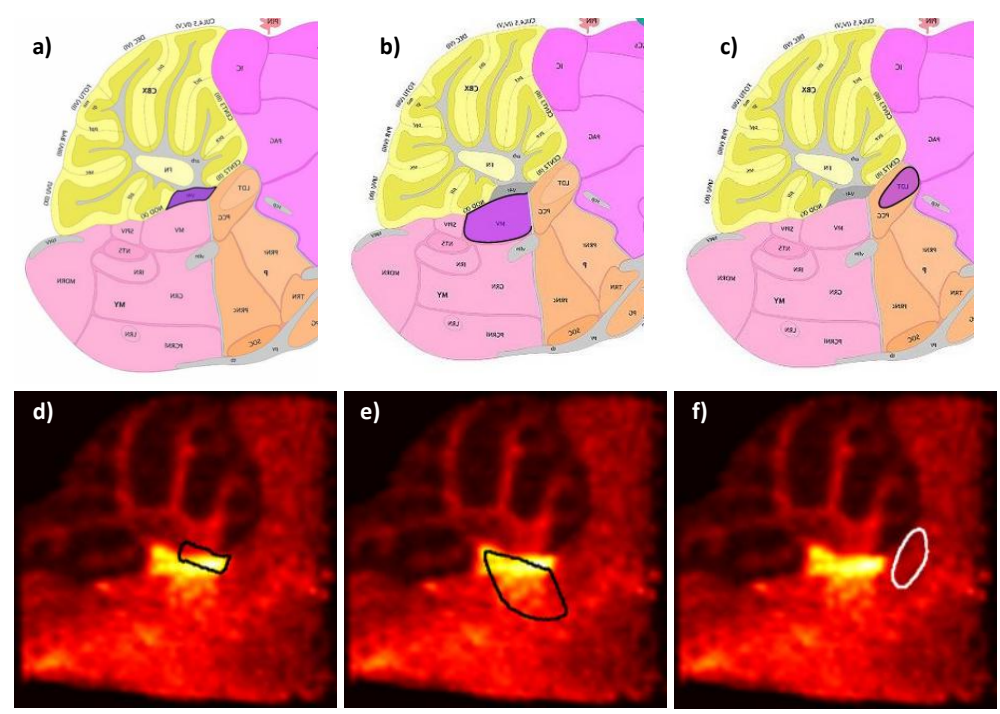

V4r lateral recess

MV

LDT

Figure 5.8 By comparing the anatomical segmentation map of the ABA reference section with the MSI data of the KO3, the hotspot can be assigned to several regions: most prominent candidates are (highlighted in violet colour): $\boldsymbol{a})$ the $4^{\text {th }}$ ventricle (V4r lateral recess), b) the medial vestibular nucleus $(M V)$, and $c)$ the laterodorsal tegmental nucleus (LDT). The accurate conclusion cannot be derived until the MSI-ABA co-registration is performed and the particular labels overlaid. Panels $\boldsymbol{d}$ )-f) show mass channel 309, fatty acid (20:1), with the respective anatomical labels shown in black or white contour. The $4^{\text {th }}$ ventricle shows by far the best match.

Note that the quality of the co-registration process is highly dependent on the quality of the experimental tissue section and on the correct choice of the ABA reference section. While the practice of MSI has improved tremendously over the past decade, there is still a fair amount of developmental work needed to refine the technique. The most important is the sample throughput. Its significant increase would allow for faster, and maybe even automated, methods development. To illustrate the time demands of a MSI experiment: the SIMS data acquisition of one of described samples took roughly 15 hours. Additional time is required for the sample preparation, data pre-processing and analysis, tissue staining, and the steps included 
in the MSI-ABA registration step. All combined, the amount of time required for a complete analysis of one sample is on the order of days.

SIMS is considered a "gentle" MS imaging method since it causes only superficial, minimal damage to a tissue surface. Even though it does not happen often, matrix-assisted laser desorption ionization (MALDI) MSI, can also damage the tissue surface as the laser impact is much higher than the impact of the primary ion beam in SIMS. Some matrices, such as 9-aminoacridine, require high laser power. The laser ablated areas are often clearly visible on the tissue after histochemical staining. Tissue with such a changed morphology is especially challenging to retrospectively co-register, let alone to determine the identity of various anatomical features. Figure 5.9 shows a comparison of a SIMS and a MALDI analyzed tissue section that was Nissl stained after the MSI experiments were completed. (Nissl) staining (and hence the histology image quality) of SIMS imaged samples can be hampered due to the gold layer often applied on top of the tissue surface. It causes a decrease in the dye intensity, and makes the co-registration challenging. The staining procedure is further complicated by the use of the ITO glass slides required for the SIMS experiments. The thin layer of ITO compensates for the charge that builds up on top of the sample during the SIMS imaging. The tissue sections have the tendency to float away from the glass surface during the staining. If not performed with special care, the tissue can be damaged during the staining protocol. (Note that the SIMS imaged tissue section of $\mathrm{KO} 2$ was damaged during the staining process. The MALDI imaged adjacent tissue section - shown in Figure 5.9b - was thus used in the $\mathrm{KO} 2$ coregistration pipeline.)
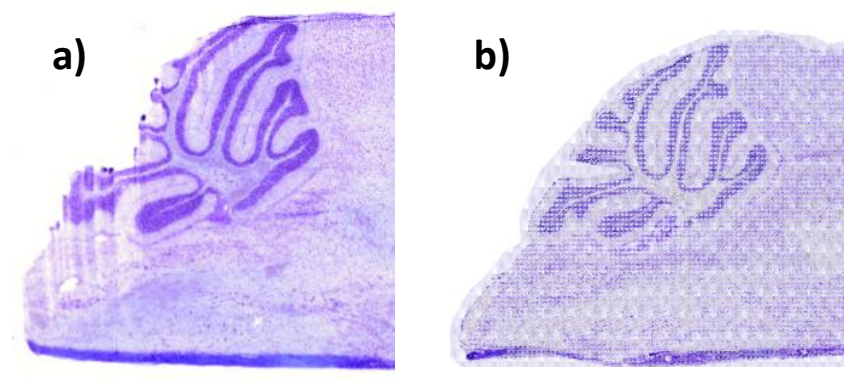

Figure 5.9 Comparison of SIMS and MALDI imaged tissue section. Whereas SIMS is considered as a non-invasive ionization technique that causes minimal damage / changes to the sample surface, the impact of laser during a MALDI experiment can significantly change tissue morphology. a) SIMS imaged Nissl stained experimental tissue section for KOI without surface changes. b) MALDI imaged Nissl stained tissue section with clearly visible imprints of the laser shots. Drawing anatomical conclusion from such a damaged tissue section is difficult if not impossible. Note that the SIMS imaged tissue section of KO2 was damaged during the staining process. The MALDI imaged adjacent tissue section was thus used in the KO2 co-registration pipeline. 
The choice of the correct $\mathrm{ABA}$ reference section is crucial to the success of the co-registration procedure. Whereas the ABA features 132 coronal mouse brain sections evenly spaced at $100 \mu \mathrm{m}$, there are only 21 sections in the sagittal plain. The sagittal sections were taken only from one hemisphere as the left and right hemispheres are anatomically symmetrical. The narrower width of the brain in sagittal plain also contributes to the lower number of the sections. Nevertheless, the sagittal sections in the ABA were taken at $200 \mu \mathrm{m}$ spacing. In practice it means that 16 twelve-micron-thick experimental tissue sections fit to only one ABA reference section. On one hand, the choice of the correct reference section is less difficult, on the other hand, it is less accurate, and thus can cause imprecision in the histology-tohistology registration step.

Accurate and reproducible sample preparation should always be at the top of the researcher's priority list. Recently a paper devoted to a good practice for tissue sectioning and general sample preparation before (MALDI) MSI was published and features several tips and tricks [182]. Sectioning with anatomical atlas as a reference is recommended especially for complex tissue samples such as brain.

Our approach assisted in the assignment of the correct anatomical label to the hotspot of FA observed in the $M f p 2^{-/}$mouse models. MFP2 is a protein that catalyzes specific reactions of the peroxisomal $\beta$-oxidation. This process is responsible for chain shortening of several carboxylate substrates, among others the VLCFA. Patients with severe MFP2 deficiency suffer from neonatal hypotonia, brain malformations, seizures, and psychomotor retardation and their life time typically does not exceed one year [234]. Similarly the KO mouse models manifest motor impairment, ataxia, and die within 6 months [234]. Oil Red O staining of the brain sections of $M f p 2^{-/}$mice showed significant lipid accumulation within several brain regions, most specifically within the ependymal cells of the whole ventricular system [229]. Dinkel et al. [235] revealed that Mfp2 is expressed in the ependymal cells isolated from the $4^{\text {th }}$ ventricle. Ependymal cells play a role in production of the cerebrospinal fluid (CSF).

We observed that in MFP2 knock-outs FA accumulate in the $4^{\text {th }}$ ventricle and in the cerebellar aqueduct. Borges et al. demonstrated in 1985 that Purkinje cells can selectively accumulate certain low- and high-molecular mass compounds from the CSF [236]. Purkinje cells provide the sole output of the cerebellum and project to the vestibular and deep cerebellar nuclei in order to control body movement and coordination. These findings suggest that in the $M f p 2^{-/}$mice the function of Purkinje cells or the related structures such as the cerebellar nuclei could be influenced by the VLCFA presence in the $4^{\text {th }}$ ventricle. However, further investigations are needed.

Because of the high impact energy of the primary ion beam in SIMS, we could assume that the observed VLCFA result from fragmentation of intact lipids. Whether 
these are glycerophospholipids, sphingolipids, triglycerides or cholesterylesters could not be determined. Whereas very long chain fatty acids with chain lengths of 22 or more carbons are selective substrates for peroxisomal $\beta$-oxidation, the shorter ones can be degraded by mitochondria and it is not clear why they accumulate in MFP2 deficient brain. As the MSI methodology does not allow quantification, it would be interesting to determine the relative contribution of each of the fatty acids by sampling CSF from the mice.

The ABA further features an application which allows for comparison of genes with similar expression pattern in terms of neuroanatomical distribution. The comparison is based on Pearson's correlation. The function produces a list of genes sorted according to their decreased correlation with the targeted gene, i.e. according to decreasing values of the Pearson's coefficient. Da Silva et al. [237] published a gene correlation study based on the ABA tool. Their targeted gene was SLC20A2 whose mutations cause the Familial idiopathic basal ganglia calcification (Fahr's disease) molecularly demonstrated as deposits of calcium in basal ganglia and other distinct brain regions. The group took the advantage of The Brain Explorer (a tool offered by the ABA) to quantitatively assess expression of the SLC20A2 gene in different brain regions. MFP2 is encoded by the $17 \beta$-hydroxysteroid dehydrogenase 4 gene $(H s d 17 b 4)$ [228]. We applied the ABA search tool to find genes correlated with $H s d 17 b 4$. The search listed the total number of 977 genes with various degrees of (anti-)correlation. Da Silva et al. used as a cut-off for the genes of interest $r \geq 0.5$ for the correlates, and $r \leq-0.5$ for the anti-correlates. We summarized the first ten hits of the correlates in Table 4.1. The search found only one gene with Pearson's coefficient lower than -0.5. The listed genes were examined through the UniProt [238] database and explanation of their function (if available) was provided. The Fatty aldehyde dehydrogenase (Aldh3a2) showed the highest correlation of all genes. It catalyzes the oxidation of long-chain aliphatic aldehydes to fatty acids, and is responsible for conversion of the sphingosine 1-phosphate degradation product hexadecenal to hexadecenoic acid [238]. It is also considered to be involved in the degradation of phytanic acid, a saturated branched chain fatty acid [239]. Another possibility is to search for genes that are expressed in particular brain regions. In our example the cells adjacent to the $4^{\text {th }}$ ventricle, i.e. ependymal cells and choroid plexus were unfortunately not included in the structural list. Further deep exploration of the genes related to MFP2 and the associated anatomical structures might provide more information on the molecular mechanism of peroxisomal dysfunctions. 


\begin{tabular}{|c|c|c|c|}
\hline Gene abbrev & Gene name & PC & Notes \\
\hline Aldh3a2 & $\begin{array}{l}\text { Aldehyde dehydrogenase family } \\
3 \text {, subfamily } A 2\end{array}$ & 0.864 & $\begin{array}{l}\text { Catalyzes the oxidation of long- } \\
\text { chain aliphatic aldehydes to fatty } \\
\text { acids. Responsible for conversion } \\
\text { of the sphingosine 1-phosphate } \\
\text { (S1P) degradation product } \\
\text { hexadecenal to hexadecenoic acid }\end{array}$ \\
\hline Atg13 & Autophagy related 13 & 0.838 & \\
\hline Brox & $\begin{array}{l}\text { BRO1 domain and CAAX motiff } \\
\text { containing }\end{array}$ & 0.832 & $\begin{array}{l}\text { Subcellular location: Lipid anchor = } \\
\text { Protein bound to the lipid bilayer } \\
\text { of a membrane through a } \\
\text { posttranslationally modification by } \\
\text { the attachment of at least one lipid } \\
\text { or fatty acid, e.g. farnesyl, } \\
\text { palmitate and myristate. }\end{array}$ \\
\hline Mphosph6 & M-phase phosphoprotein 6 & 0.814 & \\
\hline Pddc1 & $\begin{array}{l}\text { Parkinson disease } 7 \text { domain- } \\
\text { containing protein } 1\end{array}$ & 0.777 & \\
\hline Wdr5 & WD repeat domain 5 & 0.770 & \\
\hline Tacc2 & $\begin{array}{l}\text { Transforming acidic coiled-coil- } \\
\text { containing protein } 2\end{array}$ & 0.757 & \\
\hline Fam133b & Protein FAM133B & 0.751 & \\
\hline Mrps16 & $\begin{array}{l}28 \mathrm{~S} \text { ribosomal protein } \mathrm{S} 16 \text {, } \\
\text { mitochondrial }\end{array}$ & 0.741 & \\
\hline Hnrnpk & $\begin{array}{l}\text { Heterogeneous nuclear } \\
\text { ribonucleoprotein } \mathrm{K}\end{array}$ & 0.732 & \\
\hline Cacna1a & $\begin{array}{l}\text { Voltage-dependent P/Q-type } \\
\text { calcium channel subunit alpha- } \\
\text { 1A }\end{array}$ & -0.879 & $\begin{array}{l}\text { Voltage-sensitive calcium channels } \\
\text { (VSCC) mediate the entry of } \\
\text { calcium ions into excitable cells } \\
\text { and are also involved in a variety of } \\
\text { calcium-dependent processes, } \\
\text { including muscle contraction, } \\
\text { hormone or neurotransmitter } \\
\text { release, gene expression, cell } \\
\text { motility, cell division and cell } \\
\text { death. }\end{array}$ \\
\hline
\end{tabular}

Table 4.1 List of genes with a similar expression pattern (in the terms of neuroanatomical distribution) as manifests the gene Hsd17b4 (the gene encoding the MFP2 protein). The correlation calculation is based on Pearson's coefficient. The calculation was done via tool provided by the ABA. Listed are 10 most correlated genes. Cacnal a showed on the contrary high anti-correlation (the only gene with PC lower that -0.5). 


\subsection{Conclusions}

The presented work demonstrates the potential of the automated co-registration of mass spectrometry imaging (MSI) data to curated anatomical atlases for precise anatomical localization of the MSI-derived molecular signatures. We employed a fully automated pipeline to co-register brain MSI data of Mfp2 deficient mouse models to the Allen Brain Atlas (ABA). High resolution secondary ion mass spectrometry imaging (SIMS) revealed accumulation of fatty acids in specific brain regions. The advantage of high spatial resolution MSI is the enhanced similarity to histological images which improves automated co-registration. Manual comparison of SIMS images with the histological information provided by the Nissl stained tissue sections did not allow for an unequivocal annotation of the FA rich brain regions. The result of MSI-ABA co-registration linked the FA hotspot to the $4^{\text {th }}$ ventricle. The ABA tools were further used to generate a list of genes correlated with the MFP2 expression pattern. Various molecular substrates linked to the correlated genes can be used for future targeted molecular analysis of $M f p 2^{-/}$mouse models. 


\section{Epilogue: The Dynamic Knowledge}




\section{Epilogue: The Dynamic Knowledge}

\subsection{General discussion}

The brain is the control organ of our bodies. To fully understand its functioning, we need to gain solid knowledge of its structure and processes at the lowest, i.e. molecular, level. The same is valid for the understanding of the brain pathophysiology. To this time, various analytical methods have been developed to study the molecular composition of the brain. In this thesis, we presented mass spectrometry imaging (MSI) as a powerful technique to study the brain at molecular level. The presented work was aiming at the analysis of the molecules that create more than half of the brain content: the lipids. Lipids play various roles within the brain and our entire bodies. Understanding the brain lipidome thus becomes crucial in the investigation of both, the brain function and dysfunction. Owing to its high molecular specificity and relatively high spatial resolution, MSI allows us to study the brain lipidome in a great detail. MSI can thus contribute to the molecular understanding of the brain and to monitor its lipidome alterations. We must stress, however, that understanding the brain requires knowledge from many different areas and only interdisciplinary research can provide us with a broader perspective on its function. Although it is a powerful molecular imaging technique, MSI is not a universal, all-answering analytical method. However, to overcome some of its shortcomings, it is advantageously combined with other research methods as discussed in this thesis (see for example Chapter 2).

\subsubsection{Multimodal MSI of lipids}

So far, not a single MS ionization technique or protocol has been reported for ionization and detection of all lipid classes in a single experiment (nor exclusively for positive and negative ionization mode). Thus, using MSI, the complete lipidome of a sample surface can be assessed only by a combination of different MSI techniques or protocols. In this thesis, we have discussed molecular complementarity of the three most frequently employed MSI techniques: matrix-assisted laser desorption ionization (MALDI), desorption electrospray ionization (DESI), and secondary ion mass spectrometry (SIMS) (see Table 1.1 and Chapter 3).

Providing the quality of a sample surface is not compromised, a single tissue section can be imaged with different modalities. Regarding the surface damage, SIMS is a close to non-destructive ionization technique, sputtering away only nanometres of a sample's surface [240]. Thus, a SIMS imaged tissue section can be used for a 
subsequent imaging with another MS modality. We have shown this in Chapter 4 of this thesis. Similarly, Eberlin and co-workers reported on a combined DESI and MALDI imaging of a single tissue section [44]. In their report they stressed the use of solvent systems limiting tissue surface destruction during DESI experiments [44, 241]. MALDI, on the contrary, can change sample morphology, as we showed in Figure 5.9 of this thesis.

To spatially compare complementary molecular (lipid) information obtained by different MSI modalities from a single tissue section, it becomes useful to employ a system of fiducial markers which allows for a co-registration of the acquired molecular signatures. In Chapter 4, we reported on a novel methodology which allows for the spatial co-registration of MS images obtained with SIMS and MALDI performed on a single tissue section. Even though our work did not include DESI experiments, it could be assumed that DESI, too, could be capable of imaging the gold sputtered fiducial markers. In an ideal case, these fiducial markers could be implanted next to a tissue section, which would be subsequently imaged by SIMS, followed by DESI imaging and finally, after application of matrix, by MALDI MSI. Owing to the molecular complementarity of these MSI ionization techniques, such an approach would lead to an especially broad overview of a tissue lipidome (moleculome). Importantly, the spatial distribution of the lipid molecular patterns obtained by individual MSI could be compared through the co-registration enabled by the system of the fiducial markers. However, it has to be noted that before such a bold idea can be executed, two steps need to be taken: 1 . The suggestion that DESI is capable of imaging the gold sputtered fiducial markers as described in Chapter 4 has to be proven. 2. Protocols for each of the MSI technique need to be optimized to minimize the sample destruction and to maximize the molecular complementarity provided on the SIMS-DESI-MALDI axis.

The idea of multimodal imaging of lipids from a single tissue section is integrated in the diagram presented in Figure 6.1. It shows a MSI workflow that includes the methodologies presented in this thesis. The spine of the workflow is represented by graphics framed in black colour. Similarly to the Figure 1.1 shown in Chapter 1, they include sample preparation, data acquisition using MSI, data pre-processing and multivariate analysis for assessment of regional molecular differences of an analyzed sample. The basis of the pipeline further branches into two directions: 1. enhanced molecular information through multimodal MSI imaging, and 2. combination with the Allen Brain Atlas for precise anatomic annotation of the MSI derived molecular patterns. Each colour-coded branch of the diagram represents one experimental chapter of this thesis. Chapter 3 (red frames) suggests that a combination of MSI modalities increases the qualitative lipid information obtained about a sample. 
Implanting gold sputtered fiducial markers before MSI measurements, as demonstrated in Chapter 4 (green frames), allows for a co-registration of the molecular images obtained by different MSI modalities. In Chapter 5 (yellow frames), we showed how a comparison of the MSI acquired molecular data with the anatomic information provided by the Allen Brain Atlas allows for precise anatomic localization of the molecular signatures. Finally, one of the future directions of the involvement of the pipeline is marked in the graphics framed in blue. If we consider molecules beyond the lipids, protocols for MSI analysis of metabolites, peptides and proteins could be further added into the workflow. Through such a step, we could obtain a complete moleculome of specific anatomic areas of the brain. Moreover, we could spatially monitor metabolic or signalling pathways in which the detected molecules are involved [53].

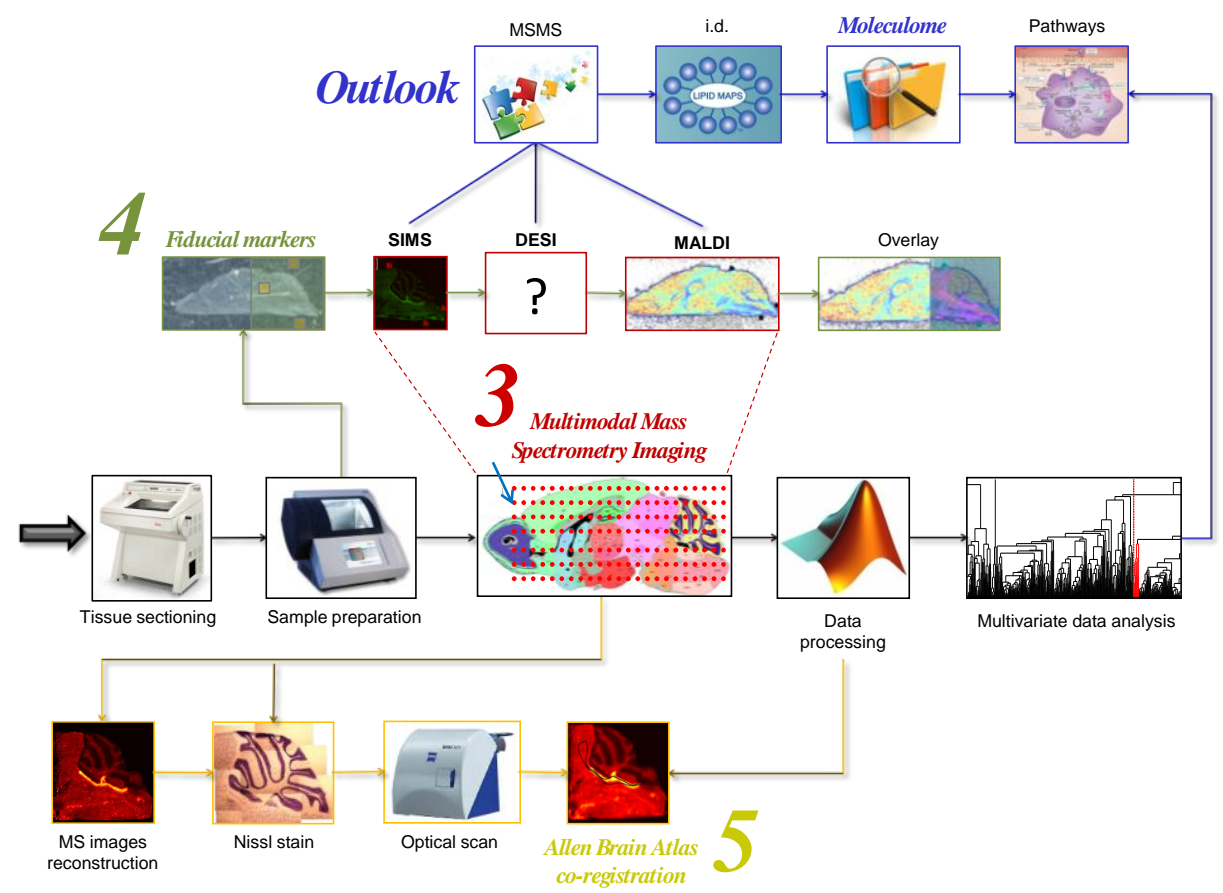

Figure 6.1 A diagram summarizing the research described in this thesis and a possible direction of its further involvement. The central theme of the workflow is represented by the "sample preparation - data acquisition - data analysis" axis (in the black frames). Each colour-coded branch of the diagram (red, green and yellow) represents one experimental chapter of this thesis (3, 4 and 5, respectively). The blue branch suggests a possible application of the protocols for obtaining information on metabolic and signalling pathways in the brain and other types of biological tissues. 
This thesis presented novel methodologies for multimodal imaging of lipids in the brain tissue. Simultaneously, however, it has generated a number of questions and possible directions of further research. I believe it is imperative for every researcher to continue generating questions. For it is the dynamism of our knowledge, not its rigidity, that allows for a constant improvement of the understanding of the world around us.

\subsubsection{Limitations of MSI}

Although big improvements were made in the MSI field since its introduction twenty years ago, the technique suffers from a number of drawbacks. Some of them, such as ionization suppression and challenges for identification of lipids, have been already mentioned in Chapter 2 and Chapter 3, respectively. Some of other limitations are briefly discussed in the following paragraphs.

\section{- Ex vivo setup}

Before (a brain) tissue sample is MS imaged, a model animal is euthanized, optionally perfused, its skull is cut open and the brain is taken out and frozen. Subsequently, thin sections of the brain are made, matrix is applied (for MALDIMSI) and then, finally, the sample is analyzed. On top of that, we have to include transport of the sample and its storage. These steps can potentially influence molecular composition of the brain tissue. For example, Dill and co-workers detected an increased number of fatty acid dimers after 24 hours of a sample's storage at room temperature [242]. These findings suggest that enzymatic processes were still present in the tissue, and thus influencing its molecular composition [242]. We can assume that also other molecular changes occur during the retrieval of the brain tissue. Thus, while the ex vivo molecular imaging provides a great deal of information about the brain, we have to bear in mind that one never knows how much the ex vivo form of the brain reflects its actual in vivo state.

- Low through-put

As demonstrated in Chapter 5, the amount of time required for a complete MSI analysis of one sample is on the order of days, if not weeks (especially if time for the actual method development is included). Thus, the room for improvement of the sample throughput in MSI analysis is huge. Its increase could allow for faster methods development and data collection. Progress has been made lately by the development of a new commercial MALDI mass spectrometers, which enable a significant (up to 50-fold) increase in speed of the data acquisition [13]. Importantly, however, faster generation of data requires improved and more efficient data management [243]. 
- Not unified protocols

Being just introduced in 1997 [9], MSI is a relatively young analytical technique. Its fast expansion in the last two decades included improvements of the instrumentation, sample preparation, and applications in a number of clinical, pharmaceutical or plant-based projects [62]. Project-targeted approaches are often employed in MSI, thus leading to a colossal amount of MSI protocols reported. For example, a plethora of various workflows has been reported for MALDI-MSI of lipids (in the brain tissue), using different matrices of various concentrations, dissolved in solvent mixtures of variable composition. In a recently published discussion note, the authors suggested a guideline for reporting the MSI protocols [244]. A database of protocols for a specific tissue type and molecular classes would become especially useful for a successful implementation of MSI into the clinical environment. 


\subsection{Summary}

Constant methodology improvement is essential in all analytical fields. In this thesis, I described novel methodologies to study lipids in the brain using multimodal mass spectrometry imaging (MSI). The described protocols improved both, the qualitative and the spatial aspects of MSI. They broadened the general workflow of the MSI analytical approach, as presented in Chapter 1.

Chapter 2 introduced the readers to the principles of MSI in greater detail. This chapter focused on the most commonly employed MSI ionization technique: matrixassisted laser desorption ionization (MALDI). The primary strength of MSI is in providing the information about the position of individual molecules within a tissue sample. However, the price to pay for the spatial information is in increased ionization suppression. Separation techniques have the ability to significantly reduce it, hence to improve ionization of low-abundant molecular species. However, due to nature of MSI, it is impossible to incorporate a separation step prior to an imaging experiment. As a result, MSI suffers from ionization suppression to a great extent. This chapter stressed that a comprehensive local sample composition information can be obtained by combination of MSI and separation techniques in a single analytical approach. We reviewed and discussed examples of MSI combined with electrophoresis, chromatography and blotting.

In Chapter 3 we introduced another MSI technique, namely desorption electrospray ionization (DESI). We employed DESI coupled to ion mobility separation (IMS) for the analysis of lipids in murine brain tissue. When coupled to MSI, IMS cannot reduce the ionization suppression as discussed in Chapter 2, since the separation occurs after the ionization process. However, it simplifies imaging data interpretation by grouping molecules based on their structure and charge. In our DESI-IMS-MSI study, we detected gangliosides, including low abundant GT and GQ species and their acetylated versions as multiply charged ions directly from the murine brain tissue. Using IMS, complex gangliosides were separated from the rest of the lipid classes based on their structure and charge state. Findings demonstrated in this chapter represent a major improvement in the spatial analysis of fragile lipid species. Gangliosides are highly abundant in the nervous system, where they play various biological roles and are involved in some brain pathologies, including lysosomal storage disorders. Knowledge of ganglioside spatial distribution can contribute to broadening our molecular understanding of the brain and its pathologies.

In this chapter, we also compared DESI imaging to MALDI-MSI experiments performed on the same ion mobility enabled instrument. We show that MALDI-IMS enabled separation of background and tissue related molecules. DESI mobilograms, 
however, contained higher number of trendlines, thus demonstrating a better separation of endogenous molecular species. We also showed that by combination of the two imaging modalities under the reported conditions, complementary lipid information is obtained. It should be noted, however, that by optimizing sample preparation or experimental conditions for both DESI and MALDI, ionization of particular lipid classes can be improved for each of the techniques. Thus far a methodology enabling MSI of all lipid classes with high sensitivity under single experimental conditions has not been reported. Consequently, it can be advantageous to utilize a combination of MSI techniques.

Finally, Chapter 3 showed examples of fragmentation spectra of various lipid species and discussed briefly the identification of lipids in the MSI analytical approach.

Secondary ion mass spectrometry (SIMS), introduced in Chapter 4, also offers complementary lipid (molecular) information when compared to MALDI. However, in order to obtain unique information about the structure and composition of tissue sections, multimodal imaging requires appropriate co-registration of different images. Consequently, this chapter focused on the problem of co-registration of MS images acquired by different modalities. We described the development and proof-ofprinciple for a novel co-registration method combining SIMS and MALDI. Our protocol is based on gold sputtered fiducial markers, implanted on and next to a tissue section prior to multimodal MSI. As SIMS has minimal mechanical impact on a sample during MSI, it is possible to re-image the same tissue section using MALDI. We showed that the markers can be visualized with SIMS and MALDI and thus enable co-registration of MS images acquired by both modalities. We showed that the method is universal and can be used for various tissue types, including brain tissue.

Chapter 5 explored the co-registration of MS images from a different perspective. MSI is often combined with other imaging modalities, such as histochemical staining, to assess anatomical information of specific tissue regions (possibly highlighted during an MSI experiment). However, direct comparison of histology based and MS based molecular images can become problematic due to potential tissue damage or changes caused by different sample preparation. On the contrary, automated comparison of MSI to curated atlases such as the Allen Brain Atlas (ABA) allows for conclusions to be drawn on the precise anatomical localization of MSI derived molecular signatures.

In this chapter we imaged brain sections of mice with an impaired lipid metabolism using SIMS. The mice were lacking a gene (MFP2) responsible for degradation of very long chain fatty acids. We found fatty acid rich deposits manifesting specific spatial patterns within the brain sections of the knockout-out 
mice. However, we were unable to unequivocally determine the anatomic localization of the SIMS-derived lipid signatures. We employed a pipeline for automated comparison of SIMS images with the Allen Brain Atlas, which resulted in a precise anatomic localization of the accumulated fatty acids. After the co-registration, the hotspots were linked to the $4^{\text {th }}$ ventricle.

The ABA contains highly standardized information on gene expression anatomically organized in a hierarchic order. We further explored the tools provided by the $\mathrm{ABA}$, and used one of them to generate a list of genes correlated with the MFP2 expression pattern. Various molecular substrates linked to the correlated genes can be used for future targeted molecular analysis of MFP2 deficient mouse models. Although the biological impact of our work is minimal, we suggested future directions of research and opened the door to further investigations. Importantly, the work presented in Chapter 5 represents an enhancement in the spatial capabilities of MSI through the automated combination with a brain anatomical atlas.

The novel protocols described in this thesis led to a proposal of a comprehensive workflow which can applied in further molecular investigations of the brain and other tissue types. The workflow, its suggested methodological improvements, and involvement in future research were described and discussed in Chapter 6. 


\subsection{Samenvatting}

Continue methodologie verbeteringen zijn essentieel in alle velden van analyse. In deze thesis beschrijf ik nieuwe methodologieën om met behulp van multimodale massa spectrometrie imaging (MSI) lipiden te bestuderen. The beschreven protocollen hebben zowel kwalitatieve als spatiele aspecten van MSI verbeterd. Zij hebben de algemene werkwijze van de MSI analytische aanpak verbreedt, zoals beschreven in Hoofdstuk 1.

Hoofdstuk 2 introduceert de lezers in de meer gedetailleerde principes van MSI. Dit hoofdstuk is gericht op de meest gebruikte MSI ionisatie techniek: matrixgeassisteerde laser desorptie ionisatie (MALDI). De voornaamste kracht van MSI ligt in het verstrekken van informatie over de locatie van individuele moleculen binnen een te onderzoeken weefsel. Echter, deze spatiele informatie gaat ten koste van het vermogen om moleculen te detecteren die in mindere mate aanwezig zijn, een fenomeen genaamd ionen onderdrukking. Scheidingtechnieken worden traditioneel ingezet om dit te overkomen. Echter, vanwege het principe van MSI is het niet mogelijk om een scheidingsstap te verwerken in een imaging experiment. In dit hoofdstuk hebben wij laten zien dat de volledige lokale moleculaire compositie kan worden achterhaald door een combinatie van MSI en scheidingstechnieken te gebruiken. We hebben hier voorbeelden van combinaties tussen MSI en elektroforese, chromatografie en blotting besproken en bediscussieerd.

In Hoofdstuk 3 hebben wij een andere MSI techniek geïntroduceerd, namelijk desorptie electrospray ionisatie (DESI). Wij hebben met DESI gekoppeld aan ionen mobiliteitsscheiding (IMS) analyses uitgevoerd van lipiden in muizen hersenweefsel. Wanneer IMS gekoppeld is aan MSI kan deze het ionen onderdrukking fenomeen, beschreven in Hoofdstuk 2, niet reduceren omdat de scheiding gebeurt na het ionisatie proces. Echter, het versimpeld de imaging data interpretatie door moleculen te groeperen op basis van hun structuur en lading. In onze DESI-IMS-MSI studie hebben wij gangliosiden inclusief lage concentraties GT en GQ moleculen en hun geacytyleerde versies in muis hersenweefsel kunnen detecteren als meervoudig geladen ionen. Dankzij IMS konden wij complexe gangliosiden scheiden van de andere lipiden soorten op basis van hun structuur en lading. Bevindingen gepresenteerd in dit hoofdstuk representeren een grote verbetering in de spatiele analyse van fragiele lipiden soorten. Gangliosiden zijn veelvoorkomend in het zenuwstelsel, waar zij diverse biologische rollen vervullen en betrokken zijn bij bepaalde hersen ziektebeelden, waaronder lysosoom opslag aandoeningen. Kennis van de spatiele verdeling van gangliosiden kan van waarde zijn in het vergroten van ons inzicht in de hersenen en hersenaandoeningen. 
In dit hoofdstuk hebben wij DESI imaging vergeleken met MALDI-MSI experimenten uitgevoerd op hetzelfde, met ionen mobiliteitsscheiding uitgeruste, instrument. Wij hebben laten zien dat het gebruik van MALDI-IMS de onderzoeker in staat stelt achtergrond en weefsel gerelateerde moleculen van elkaar te scheiden. DESI mobilograms bevatten echter meer trendlijnen, wat duidt op een betere scheiding van endogene moleculaire soorten. Wij hebben laten zien dat de combinatie van de beide imaging modaliteiten onder de gerapporteerde condities complementaire informatie levert. Daarbij moet worden opgemerkt dat door het optimaliseren van monster voorbereiding en experimentele condities voor zowel DESI als MALDI de ionisatie efficiëntie kan worden verhoogd. Tot dusverre is er nog geen methodologie met hoge gevoeligheid voor alle lipiden soorten onder gelijke experimentele condities gerapporteerd. Het gebruik van een combinatie van MSI technieken kan dus voordelig zijn.

Tot slot presenteert Hoofdstuk 3 voorbeelden van fragmentatie spectra van verschillende lipiden soorten en wordt de identificatie van lipiden in de MSI analytische werkwijze bediscussieerd.

Secundaire ionen massa spectrometrie (SIMS), geïntroduceerd in Hoofdstuk 4, kan additionele moleculaire informatie leveren over lipiden, in aanvulling op MALDI. Echter, om unieke informatie te verkrijgen over de structuur en compositie van weefsel coupes dienen de beelden verkregen uit meerdere MSI modaliteiten correct te worden gecombineerd. Daarom is dit hoofdstuk gecentreerd rondom het probleem van co-registratie van datasets verkregen uit verschillende MSI modaliteiten. Wij hebben de ontwikkeling van een nieuwe methode om MSI datasets te co-registreren beschreven en gedemonstreerd met SIMS en MALDI data. Ons protocol is gebaseerd op referentiemarkeringen die met een goud bundel zijn aangebracht naast en op een weefsel coupes in voorbereiding op multimodale MSI experimenten. Omdat SIMS minimale mechanische impact heeft op een monster tijdens een MSI experiment is het mogelijk om hetzelfde weefsel opnieuw te analyseren met MALDI. We hebben aangetoond dat de markeringen kunnen worden gevisualiseerd met SIMS en MALDI en dus de co-registratie van MSI datasets mogelijk maken. Wij hebben laten zien dat de methode universeel is en dus kan worden gebruikt voor allerlei weefsel types, waaronder dus ook hersenweefsel.

In Hoofdstuk 5 is de co-registratie van MSI beelden verkend vanuit een ander perspectief. MSI wordt vaak gecombineerd met andere imaging modaliteiten, zoals immuunhistochemische kleuring, om anatomische informatie van specifieke weefselregionen (mogelijk gestuurd door MSI resultaten) te beoordelen. Echter kan directe vergelijking van op histologie en MS gebaseerde beelden problematisch zijn door eventuele schade of veranderingen aan het weefsel als gevolg van monster 
voorbereiding. Tegelijkertijd maakt geautomatiseerde vergelijking van MSI beelden met anatomische atlassen zoals de Allen Brain Atlas (ABA) het mogelijk om conclusies te trekken over exacte anatomische lokalisatie van moleculaire verdelingen geobserveerd in MSI experimenten.

In dit hoofdstuk hebben wij met SIMS MSI beelden gemaakt van hersencoupes van muizen met vertraagd lipiden metabolisme. Bij deze muizen ontbrak het MFP2 gen dat verantwoordelijk is voor de afbraak van zeer lange vetzuren. Wij hebben in de hersencoupes van deze muizen vetrijke afzettingen geobserveerd in specifieke spatiele patronen. Wij waren echter niet in staat om ondubbelzinnig aan te tonen waar deze SIMS-afgeleide lipiden patronen anatomisch gelokaliseerd waren. Met behulp van een protocol voor automatische vergelijking van SIMS images met de Allen Brain Atlas waren in staat de samengeklonterde vetzuren anatomisch te lokaliseren. $\mathrm{Na}$ de co-registratie konden wij deze plaatsen in het vierde ventrikel. Gebruikmakend van andere mogelijkheden van de Allen Brain Atlas konden wij een lijst met aan het MFP2 expressie patroon gecorreleerde genen opstellen. Verscheidene moleculaire substraten die gekoppeld zijn aan de gecorreleerde genen kunnen worden gebruikt voor toekomstige gerichte moleculaire analyse van MFP2 gen missende muismodellen. Hoewel de biologische waarde van ons onderzoek minimaal is hebben wij toekomstige onderzoeksrichtingen voorgesteld en een basis gemaakt voor aanvullend onderzoek. Vooral representeert het onderzoek getoond in Hoofdstuk 5 een verhoging van de spatiele mogelijkheden van MSI door de geautomatiseerde combinatie met een anatomische atlas.

De nieuwe protocollen beschreven in deze thesis hebben geleidt tot een voorstel voor een veelomvattende werkwijze die toegepast kan worden in verdere moleculaire onderzoeken naar hersen- en ander weefsel. Deze werkwijze, de methodologische verbeteringen die hierbij voorgesteld worden en de toepassing in toekomstig onderzoek is beschreven en bediscussieerd in Hoofdstuk 6. 


\subsection{Valorisation}

The brain is the control organ of our bodies and the desire to understand its organization and functioning is as old as mankind. A number of ongoing international brain research projects, such as The Human Brain Project [245], The Allen Brain Atlas [196] or The BRAIN Initiative [246], prove that brain research has been receiving particular attention in the past decade. These ambitious projects are focused on elucidating how the brain functions and "how it enables the human body to record, process, utilize, store, and retrieve information" [246]. They also target pathological states of the brain and are "seeking new ways of treating, curing, and even preventing brain disorders." [246] Maladies such as neurodegenerative diseases, mental disorders and stroke are, indeed, of great clinical importance. Many of these disorders are currently incurable. As such, they have a great impact not only on the quality of life of the patients and their families but also represent a significant economic burden for the society [29].

The brain's biochemical processes are dependent on mutual interactions and spatiotemporal translocations of molecules [5, 64]. Various chemical entities are also known to be involved in some brain disorders including depression (decrease of serotonin), Parkinson's disease (decrease of dopamine), Alzheimer's disease (decrease of acetylcholine and accumulation of excitatory amino acids such as glutamic acid, GABA, aspartic acid and glycin) or schizophrenia (increase of dopamine). Hence, in order to better understand the brain and the processes underlying its pathological states, it is imperative to gain more knowledge of the brain at the molecular level.

To this date, various analytical methods have been developed to study the molecular composition of the brain. Among them, molecular neuroimaging has the unique capability of providing simultaneous information on the identity and position of molecules within the brain. The major challenge in molecular neuroimaging is to achieve high molecular specificity at high spatial resolution. Mass spectrometry imaging (MSI) is a relatively young molecular imaging technique which addresses both of these aspects with a particular success.

In this thesis, I presented MSI as a powerful technique to study the brain at the molecular level, with the emphasis on lipids. Brain and the central nervous system (CNS) are extremely rich in lipids, which play structural roles and also participate in many biochemical processes of the CNS [29]. On one hand, changes in lipid metabolism can significantly influence brain function (for example, the case in lysosomal storage disorders such as Niemann-Pick disease [24]). On the other hand, many brain pathologies have been shown to be accompanied by qualitative, quantitative or spatial alterations of the lipids within the brain [25, 28]. For example, 
links between lipids and the pathological mechanism of several neurodegenerative disorders have been described: It was shown that cholesterol contributes to the formation of amyloid plaques in the brains of Alzheimer's disease patients [25] and the same role was attributed to docosahexaenoic acid during Parkinson's disease [247].

Owing to its high molecular specificity and relatively high spatial resolution, MSI allows us to study the brain lipidome in a great detail. For example, MSI was employed to study not only cholesterol distribution [45, 60] but also global phospholipid changes [59] in the brains of Alzheimer's disease patients and transgenic mouse models of the disease. Results of these studies confirm the role of MSI as a powerful tool for the investigation of brain's lipidome (and its entire "moleculome").

Constant improvement in methodology is essential in all scientific fields, including mass spectrometry (imaging). In this thesis I described novel methods for MSI of lipids in the brain tissue. The protocols improved both, the qualitative and the spatial aspects of MSI. Moreover, they led to a proposal of a comprehensive workflow that can be applied in further molecular investigation of the brain and partly other tissue types. This suggested workflow can ultimately contribute to the molecular understanding of the brain and to monitor lipidome alterations related to its pathological states.

The work presented in this thesis is of interest to researchers working in biomedical fields, including neuroscientists involved in research of the molecular pathology of brain disorders, such as Alzheimer's disease or neuropsychiatric disorders. MSI can complement analytical methodologies routinely employed in neuroresearch. Thus, it can offer a deeper understanding of the changes in the molecular organization of the brain caused by its pathological states. Furthermore, researchers involved in the investigation of lipids and mass spectrometry (imaging) will also benefit from the new protocols described herein. The work reported in this thesis includes collaborative studies involving research groups specialized in different fields of science. These include groups devoted to instrumentation development, software development (image co-registration) and the study of lipid metabolism disorders. By linking a broad range of expertise, this thesis presents novel analytical approaches and the software capability to deliver practical and powerful analytical tools. 
Appendix 


\section{Appendix}

\subsection{Bibliography}

1. Mosso, A., Sulla circolazione del sangue nel cervello dell'uomo. Atti. R. Accad. Lincei Mem. Cl. Sci. Fis. Mat. Nat., 1880. III: p. 237-358.

2. $\quad$ Sandrone, S., et al., Weighing brain activity with the balance: Angelo Mosso's original manuscripts come to light. Brain, 2014. 137(Pt 2): p. 621-33.

3. McNamara, R.K., Deciphering the role of docosahexaenoic acid in brain maturation and pathology with magnetic resonance imaging. Prostaglandins Leukot Essent Fatty Acids, 2013. 88(1): p. 33-42.

4. De Carlos, J.A. and J. Borrell, A historical reflection of the contributions of Cajal and Golgi to the foundations of neuroscience. Brain Res. Rev., 2007. 55(1): p. 8-16.

5. Shariatgorji, M., P. Svenningsson, and P.E. Andren, Mass spectrometry imaging, an emerging technology in neuropsychopharmacology. Neuropsychopharmacology, 2014. 39(1): p. 34-49.

6. Thomson, J.J., XXVI. Rays of positive electricity. Philosophical Magazine Series 6, 1911. 21(122): p. 225-249.

7. Karas, M., D. Bachmann, and F. Hillenkamp, Influence of the wavelength in highirradiance ultraviolet laser desorption mass spectrometry of organic molecules. Anal. Chem., 1985. 57(14): p. 2935-2939.

8. $\quad$ Fenn, J.B., et al., Electrospray ionization for mass spectrometry of large biomolecules. Science, 1989. 246(4926): p. 64-71.

9. $\quad$ Caprioli, R.M., T.B. Farmer, and J. Gile, Molecular imaging of biological samples: localization of peptides and proteins using MALDI-TOF MS. Anal. Chem., 1997. 69(23): p. 4751-4760.

10. Alexandrov, T., MALDI imaging mass spectrometry: statistical data analysis and current computational challenges. BMC Bioinformatics, 2012. 13 Suppl 16: p. S11.

11. Jones, E.A., et al., Imaging mass spectrometry statistical analysis. J. Proteom., 2012. 75(16): p. 4962-4989.

12. Veloso, A., et al., Anatomical distribution of lipids in human brain cortex by imaging mass spectrometry. J Am Soc Mass Spectrom, 2011. 22(2): p. 329-38.

13. Ogrinc Potocnik, N., et al., Use of advantageous, volatile matrices enabled by nextgeneration high-speed matrix-assisted laser desorption/ionization time-of-flight imaging employing a scanning laser beam. Rapid Commun Mass Spectrom, 2015. 29(23): p. 2195-203.

14. Yao, I., et al., In situ proteomics with imaging mass spectrometry and principal component analysis in the Scrapper-knockout mouse brain. Proteomics, 2008. 8(18): p. 3692-701.

15. Alexandrov, T. and J.H. Kobarg, Efficient spatial segmentation of large imaging mass spectrometry datasets with spatially aware clustering. Bioinformatics, 2011. 27(13): p. i230-i238.

16. Alexandrov, T., et al., Super-resolution segmentation of imaging mass spectrometry data: Solving the issue of low lateral resolution. Journal of Proteomics 2011. 75: p. 237-245.

17. Deininger, S.r.-O., et al., MALDI Imaging Combined with Hierarchical Clustering as a New Tool for the Interpretation of Complex Human Cancers. Journal of Proteome Research, 2008. 7(12): p. 5230-5236. 
18. Mascini, N.E., et al., The use of mass spectrometry imaging to predict treatment response of patient-derived xenograft models of triple-negative breast cancer. $\mathrm{J}$ Proteome Res, 2015. 14(2): p. 1069-75.

19. Shevchenko, A. and K. Simons, Lipidomics: coming to grips with lipid diversity. Nat Rev Mol Cell Biol, 2010. 11(8): p. 593-8.

20. http://www.lipidmaps.org/.

21. Fahy, E., et al., A comprehensive classification system for lipids. J Lipid Res, 2005. 46(5): p. 839-61.

22. Bazan, N.G., Lipid signaling in neural plasticity, brain repair, and neuroprotection. Mol Neurobiol, 2005. 32(1): p. 89-103.

23. Mistry, P.K., et al., Understanding the natural history of Gaucher disease. Am J Hematol, 2015. 90 Suppl 1: p. S6-11.

24. Schuchman, E.H. and M.P. Wasserstein, Types A and B Niemann-Pick disease. Best Pract Res Clin Endocrinol Metab, 2015. 29(2): p. 237-47.

25. Puglielli, L., R.E. Tanzi, and D.M. Kovacs, Alzheimer's disease: the cholesterol connection. Nat Neurosci, 2003. 6(4): p. 345-51.

26. Chan, R.B., et al., Comparative lipidomic analysis of mouse and human brain with Alzheimer disease. J Biol Chem, 2012. 287(4): p. 2678-88.

27. Grela, A., et al., Application of fatty acid and lipid measurements in neuropsychiatry. Clin Chem Lab Med, 2015.

28. Muller, C.P., et al., Brain membrane lipids in major depression and anxiety disorders. Biochim Biophys Acta, 2015. 1851(8): p. 1052-65.

29. Adibhatla, R.M. and J.F. Hatcher, Role of Lipids in Brain Injury and Diseases. Future lipidology, 2007. 2(4): p. 403-422.

30. Paglia, G., et al., Ion mobility-derived collision cross section as an additional measure for lipid fingerprinting and identification. Anal Chem, 2015. 87(2): p. 113744.

31. Ellis, S.R., et al., Surface analysis of lipids by mass spectrometry: more than just imaging. Prog Lipid Res, 2013. 52(4): p. 329-53.

32. Dawson, G., Measuring brain lipids. Biochim Biophys Acta, 2015.

33. Almeida, R., et al., Quantitative spatial analysis of the mouse brain lipidome by pressurized liquid extraction surface analysis. Anal Chem, 2015. 87(3): p. 1749-56.

34. Cimino, J., et al., Towards lipidomics of low-abundant species for exploring tumor heterogeneity guided by high-resolution mass spectrometry imaging. Int J Mol Sci, 2013. 14(12): p. 24560-80.

35. Mehlem, A., et al., Imaging of neutral lipids by oil red $O$ for analyzing the metabolic status in health and disease. Nat Protoc, 2013. 8(6): p. 1149-54.

36. Brown, W.J., T.R. Sullivan, and P. Greenspan, Nile red staining of lysosomal phospholipid inclusions. Histochemistry, 1992. 97(4): p. 349-54.

37. Delikatny, E.J., et al., MR-visible lipids and the tumor microenvironment. NMR Biomed, 2011. 24(6): p. 592-611.

38. Shanta, S.R., et al., Global changes in phospholipids identified by MALDI MS in rats with focal cerebral ischemia. J Lipid Res, 2012. 53(9): p. 1823-31.

39. Roux, A., et al., Chronic ethanol consumption profoundly alters regional brain ceramide and sphingomyelin content in rodents. ACS Chem Neurosci, 2015. 6(2): p. 247-59.

40. Cerruti, C.D., et al., MALDI imaging and structural analysis of rat brain lipid negative ions with 9-aminoacridine matrix. Anal Chem, 2012. 84(5): p. 2164-71.

41. Chughtai, K., et al., Mass spectrometry images acylcarnitines, phosphatidylcholines, and sphingomyelin in MDA-MB-231 breast tumor models. J Lipid Res, 2013. 54(2): p. 333-44. 
42. $\quad$ Manicke, N.E., et al., Desorption electrospray ionization (DESI) mass spectrometry and tandem mass spectrometry (MS/MS) of phospholipids and sphingolipids: ionization, adduct formation, and fragmentation. J Am Soc Mass Spectrom, 2008. 19(4): p. 531-43.

43. Dill, A.L., et al., Mass spectrometric imaging of lipids using desorption electrospray ionization. J Chromatogr B Analyt Technol Biomed Life Sci, 2009. 877(26): p. 28839.

44. Eberlin, L.S., et al., Desorption electrospray ionization then MALDI mass spectrometry imaging of lipid and protein distributions in single tissue sections. Anal Chem, 2011. 83(22): p. 8366-71.

45. Lazar, A.N., et al., Time-of-flight secondary ion mass spectrometry (TOF-SIMS) imaging reveals cholesterol overload in the cerebral cortex of Alzheimer disease patients. Acta Neuropathol, 2013. 125(1): p. 133-44.

46. Debois, D., et al., In situ lipidomic analysis of nonalcoholic fatty liver by cluster TOF-SIMS imaging. Anal Chem, 2009. 81(8): p. 2823-31.

47. Sjovall, P., J. Lausmaa, and B. Johansson, Mass spectrometric imaging of lipids in brain tissue. Anal Chem, 2004. 76(15): p. 4271-8.

48. Passarelli, M.K. and N. Winograd, Lipid imaging with time-of-flight secondary ion mass spectrometry (ToF-SIMS). Biochim Biophys Acta, 2011. 1811(11): p. 976-90.

49. Amstalden van Hove, E.R., et al., Multimodal mass spectrometric imaging of small molecules reveals distinct spatio-molecular signatures in differentially metastatic breast tumor models. Cancer Res, 2010. 70(22): p. 9012-21.

50. Eberlin, L.S., DESI-MS imaging of lipids and metabolites from biological samples. Methods Mol Biol, 2014. 1198: p. 299-311.

51. Murphy, R.C., J.A. Hankin, and R.M. Barkley, Imaging of lipid species by MALDI mass spectrometry. J Lipid Res, 2009. 50 Suppl: p. S317-22.

52. Berry, K.A., et al., MALDI imaging of lipid biochemistry in tissues by mass spectrometry. Chem Rev, 2011. 111(10): p. 6491-512.

53. Dekker, T.J., et al., Towards imaging metabolic pathways in tissues. Anal Bioanal Chem, 2015. 407(8): p. 2167-76.

54. Spraggins, J.M., et al., MALDI FTICR IMS of Intact Proteins: Using Mass Accuracy to Link Protein Images with Proteomics Data. J Am Soc Mass Spectrom, 2015. 26(6): p. 974-85.

55. Muller, L., et al., Lipid imaging within the normal rat kidney using silver nanoparticles by matrix-assisted laser desorption/ionization mass spectrometry. Kidney Int, 2015. 88(1): p. 186-92.

56. Chan, K., et al., MALDI mass spectrometry imaging of gangliosides in mouse brain using ionic liquid matrix. Analytica Chimica Acta, 2009. 639(1-2): p. 57-61.

57. Angel, P.M., et al., Enhanced sensitivity for high spatial resolution lipid analysis by negative ion mode MALDI imaging mass spectrometry. Anal. Chem., 2012. 84(3): p. 1557-1564.

58. Kettling, H., et al., MALDI mass spectrometry imaging of bioactive lipids in mouse brain with a Synapt G2-S mass spectrometer operated at elevated pressure: improving the analytical sensitivity and the lateral resolution to ten micrometers. Anal Chem, 2014. 86(15): p. 7798-805.

59. Hong, J.H., et al., Global changes of phospholipids identified by MALDI imaging mass spectrometry in a mouse model of Alzheimer's disease. J Lipid Res, 2015.

60. Sole-Domenech, S., et al., Localization of cholesterol, amyloid and glia in Alzheimer's disease transgenic mouse brain tissue using time-of-flight secondary ion mass spectrometry (ToF-SIMS) and immunofluorescence imaging. Acta Neuropathol, 2013. 125(1): p. 145-57. 
61. Matsumoto, J., et al., Abnormal phospholipids distribution in the prefrontal cortex from a patient with schizophrenia revealed by matrix-assisted laser desorption/ionization imaging mass spectrometry. Anal Bioanal Chem, 2011. 400(7): p. 1933-43.

62. Chughtai, K. and R.M. Heeren, Mass spectrometric imaging for biomedical tissue analysis. Chem Rev, 2010. 110(5): p. 3237-77.

63. Aichler, M. and A. Walch, MALDI Imaging mass spectrometry: current frontiers and perspectives in pathology research and practice. Lab Invest, 2015. 95(4): p. 422-31.

64. Hanrieder, J., et al., Imaging Mass Spectrometry in Neuroscience. ACS Chem. Neurosci., 2013. 4(5): p. 666-679.

65. Skraskova, K., et al., Precise Anatomic Localization of Accumulated Lipids in Mfp2 Deficient Murine Brains Through Automated Registration of SIMS Images to the Allen Brain Atlas. J Am Soc Mass Spectrom, 2015. 26(6): p. 948-57.

66. Thomas, A., et al., Histology-driven data mining of lipid signatures from multiple imaging mass spectrometry analyses: application to human colorectal cancer liver metastasis biopsies. Anal Chem, 2013. 85(5): p. 2860-6.

67. Longuespee, R., et al., Proteomic analyses of serous and endometrioid epithelial ovarian cancers - cases studies - molecular insights of a possible histological etiology of serous ovarian cancer. Proteomics Clin Appl, 2013. 7(5-6): p. 337-54.

68. Hillenkamp, F., et al., Matrix-assisted laser desorption/ionization mass spectrometry of biopolymers. Anal. Chem., 1991. 63(24): p. 1193-1203.

69. Dreisewerd, K., The desorption process in MALDI. Chem. Rev., 2003. 103(2): p. 395-426.

70. Karas, M. and R. Kruger, Ion formation in MALDI: the cluster ionization mechanism. Chem. Rev., 2003. 103(2): p. 427-440.

71. Ellis, S.R., et al., Surface analysis of lipids by mass spectrometry: more than just imaging. Prog. Lipid Res., 2013. 24(4): p. 329-353.

72. Knochenmuss, R. and R. Zenobi, MALDI ionization: The role of in-plume processes. Chem. Rev., 2003. 103(2): p. 441-452.

73. Luxembourg, S.L., et al., Effect of Local Matrix Crystal Variations in MatrixAssisted Ionization Techniques for Mass Spectrometry. Anal. Chem., 2003. 75(10): p. 2333-2341.

74. Yang, J. and R.M. Caprioli, Matrix Pre-Coated Targets for Direct Lipid Analysis and Imaging of Tissue. Anal. Chem., 2013. 85(5): p. 2907-2912.

75. Chaurand, P., Imaging mass spectrometry of thin tissue sections: A decade of collective efforts. Journal of Proteomics, 2012. 75(16): p. 4883-4892.

76. Chaurand, P., S.A. Schwartz, and R.M. Caprioli, Imaging mass spectrometry: a new tool to investigate the spatial organization of peptides and proteins in mammalian tissue sections. Curr. Opin. Chem. Biol. , 2002. 6(5): p. 676-681.

77. Amstalden, E.R., D.F. Smith, and R.M.A. Heeren, A concise review of Imaging Mass Spectrometry. J. Chromatogr. A, 2010. 1217(25): p. 3946-3954.

78. Chughtai, K. and R.M.A. Heeren, Mass Spectrometric Imaging for biomedical tissue analysis. Chem. Rev., 2010. 110(5): p. 3237-3277.

79. Wall, D.B., et al., Continuous sample deposition from reversed-phase liquid chromatography to tracks on a matrix-assisted laser desorption/ionization precoated target for the analysis of protein digests. Electrophoresis 2002. 23(18): p. 3193-3204.

80. Zhang, Z., C. Jia, and L. Li, Neuropeptide analysis with liquid chromatographycapillary electrophoresis-mass spectrometric imaging. J. Sep. Sci., 2012. 35(14): p. 1779-1784.

81. Vaezzadeh, A.R., et al., Imaging mass spectrometry using peptide isoelectric focusing. Rapid Commun. Mass Spectrom., 2008. 22(17): p. 2667-2676. 
82. McComb, M.E., et al., Use of a non-porous polyurethane membrane as a sample support for matrix-assisted laser desorption/ionization time-of-flight mass spectrometry of peptides and proteins. Rapid Commun. Mass Spectrom., 1997. 11(15): p. 1716-1722.

83. Strupat, K., et al., Matrix-assisted laser desorption/ionization mass spectrometry of proteins electroblotted after polyacrylamide gel electrophoresis. Anal. Chem. , 1994. 66: p. 464-470.

84. Chaurand, P., M. Stoeckli, and R.M. Caprioli, Direct profiling of proteins in biological tissue sections by MALDI mass spectrometry. Anal. Chem. , 1999. 71(23): p. 5263-5270.

85. Jungblut, P. and B. Thiede, Protein identification from 2-DE gels by MALDI mass spectrometry. Mass Spectrom. Rev., 1997. 16(3): p. 145-162.

86. Blackledge, J.A. and A.J. Alexander, Polyethylene membrane as a sample support for direct matrix-assisted laser desorption/ionization mass spectrometric analysis of high mass proteins. Anal. Chem., 1995. 67(5): p. 843-848.

87. Worrall, T.A., R.J. Cotter, and A.S. Woods, Purification of contaminated peptides and proteins on synthetic membrane surfaces for matrix-assisted laser desorption/ionization mass spectrometry. Anal. Chem., 1998. 70(4): p. 750-756.

88. McComb, M.E., et al., Characterization of hemoglobin variants by MALDI-TOF MS using a polyurethane membrane as the sample support. Anal. Chem. , 1998. 70(24): p. 5142-5149.

89. Zaluzec, E.J., et al., Direct matrix-assisted laser desorption ionization mass spectrometric analysis of proteins immobilized on nylon-based membranes. J. Am. Soc Mass Spectrom. , 1994. 5(4): p. 230-237.

90. Mock, K.K., C.W. Sutton, and J.S. Cottrell, Sample immobilization protocols for matrix-assisted laser-desorption mass spectrometry. Rapid Commun. Mass Spectrom., 1992. 9(6): p. 233-238.

91. Zhang, H. and R.M. Caprioli, Direct analysis of aqueous samples by matrix-assisted laser desorption ionization mass spectrometry using membrane targets precoated with matrix. J. Mass Spectrom., 1996. 31(6): p. 690-692.

92. Scherl, A., et al., Gold coating of non-conductive membranes before matrix-assisted laser desorption/ionization tandem mass spectrometric analysis prevents charging effect. Rapid Commun. Mass Spectrom., 2005. 19(5): p. 605-610.

93. Patterson, S.D. and R. Aebersold, Mass spectrometric approaches for the identification of gel-separated proteins. Electrophoresis, 1995. 16(10): p. 1791-1814.

94. Miliotis, T., et al., Ready-made matrix-assisted laser desorption/ionization target plates coated with thin matrix layer for automated sample deposition in high-density array format. Rapid Commun. Mass Spectrom. , 2002. 16(2): p. 117-126.

95. Miliotis, T., et al., Capillary liquid chromatography interfaced to matrix-assisted laser desorption/ionization time-of-flight mass spectrometry using an on-line coupled piezoelectric flow-through microdispenser. J. Mass Spectrom., 2000. 35(3): p. 369377.

96. Schuerenberg, M., et al., Prestructured MALDI-MS sample supports. Anal. Chem. , 2000. 72(15): p. 3436-3442.

97. Goodwin, R.J.A., Sample preparation for mass spectrometry imaging: small mistakes can lead to big consequences. J. Proteom., 2012. 75(16): p. 4893-4911.

98. Shariatgorji, M., et al., Controlled-pH tissue cleanup protocol for signal enhancement of small molecule drugs analyzed by MALDI-MS imaging. Anal. Chem., 2012. 84(10): p. 4603-4607. 
99. Amstalden van Hove, E.R., et al., An alternative paper based tissue washing method for mass spectrometry imaging: localized washing and fragile tissue analysis. J. Am. Soc. Mass Spectrom., 2011. 22(10): p. 1885-1890.

100. Weidner, S., P. Knappe, and U. Panne, MALDI-TOF imaging mass spectrometry of artifacts in "dried droplet" polymer samples. Anal. Bioanal. Chem., 2011. 401(1): p. 127-134.

101. Weidner, S.M. and J. Falkenhagen, Imaging mass spectrometry for examining localization of polymeric composition in matrix-assisted laser desorption/ionization samples. Rapid Commun. Mass Spectrom., 2009. 23(5): p. 653-660.

102. Celis, J.E. and P. Gromov, $2 D$ protein electrophoresis: can it be perfected? Curr. Opin. Biotech., 1999. 10(1): p. 16-21.

103. Walker, A.K., G. Rymar, and P.C. Andrews, Mass spectrometric imaging of immobilized $\mathrm{pH}$ gradient gels and creation of "virtual" two-dimensional gels. Electrophoresis, 2001. 22(5): p. 933-945.

104. Ogorzalek Loo, R.R., et al., Mass spectrometry of proteins directly from polyacrylamide gels. Anal. Chem., 1996. 68(11): p. 1910-1917.

105. Yang, Y., et al., Development of an integrated approach for evaluation of 2-D gel image analysis: impact of multiple proteins in single spots on comparative proteomics in conventional 2-D gel/MALDI workflow. Electrophoresis, 2007. 28(12): p. 2080-2094.

106. Loo, R.R., et al., Visualization and Analysis of the E. coli Proteome by Mass Spectrometry. Anal. Chem. , 2001. 73: p. 4063-4070.

107. Gusev, A.I., Interfacing matrix-assisted laser desorption/ionization mass spectrometry with column and planar separations. Fresenius J. Anal. Chem. , 2000. 366(6-7): p. 691-700.

108. Poole, C.F., Thin-layer chromatography: challenges and opportunities. J. Chromatogr. A, 2003. 1000(1-2): p. 963-984.

109. Tuzimski, T., Application of different modes of thin-layer chromatography and mass spectrometry for the separation and detection of large and small biomolecules. $\mathrm{J}$. Chromatogr. A, 2011. 1218(49): p. 8799-8812.

110. Fuchs, B., R. Sus, and J. Schiller, An update of MALDI-TOF mass spectrometry in lipid research. Prog. Lipid Res., 2010. 49(1): p. 450.

111. Fuchs, B., et al., Lipid analysis by thin-layer chromatography--a review of the current state. J. Chromatogr. A, 2011. 1218(19): p. 2754-2774.

112. Goto-Inoue, N., et al., High-sensitivity analysis of glycosphingolipids by matrixassisted laser desorption/ionization quadrupole ion trap time-of-flight imaging mass spectrometry on transfer membranes. J. Chromatogr. B 2008. 870(1): p. 74-83.

113. Rohlfing, A., et al., IR-MALDI-MS analysis of HPTLC - Separated phospholipid mixtures directly from the TLC plate. Analytical Chemistry, 2007. 79(15): p. 57935808.

114. Van Berkel, G.J. and V. Kertesz, Automated sampling and imaging of analytes separated on thin-layer chromatography plates using desorption electrospray ionization mass spectrometry. Anal. Chem., 2006. 78(14): p. 4938-4944.

115. Pasilis, S.P., et al., HPTLC/DESI-MS imaging of tryptic protein digests separated in two dimensions. J. Mass Spectrom., 2008. 43(12): p. 1627-1635.

116. Chen, Y.C., J. Shiea, and J. Sunner, Thin-layer chromatography-mass spectrometry using activated carbon, surface-assisted laser desorption/ionization. J. Chromatogr., 1998. A 826(1): p. 77-86.

117. Fanibanda, T., J. Milnes, and J. Gormally, Thin layer chromatography-mass spectrometry using infrared lase desorption. Int. J. Mass Spectrom. Ion. Proc, 1994. 140: p. $127-132$. 
118. Kuwayama, K., et al., Rapid, simple, and highly sensitive analysis of drugs in biological samples using thin-layer chromatography coupled with matrix-assisted laser desorption/ionization mass spectrometry. Anal. Bioanal. Chem. , 2012. 402(3): p. 1257-1267.

119. Fuchs, B., et al., Phosphatidylcholines and-ethanolamines can be easily mistaken in phospholipid mixtures: a negative ion MALDI-TOF MS study with 9-aminoacridine as matrix and egg yolk as selected example. Anal. Bioanal. Chem., 2009. 395(8): p. 2479-2487.

120. Ivleva, V.B., et al., Ganglioside Analysis by Thin-Layer Chromatography MatrixAssisted Laser Desorption/Ionization Orthogonal Time-of-Flight Mass Spectrometry. J. Am. Soc. Mass Spectrom., 2005. 16(9): p. 1552-1560.

121. Ivleva, V.B., et al., Coupling Thin-Layer Chromatography with Vibrational Cooling Matrix-Assisted Laser Desorption/Ionization Fourier Transform Mass Spectrometry for the Analysis of Ganglioside Mixtures. Analytical Chemistry, 2004. 76(21): p. 6484-6491.

122. Nimptsch, K., et al., Differently complex oligosaccharides can be easily identified by matrix-assisted laser desorption and ionization time-of-flight mass spectrometry directly from a standard thin-layer chromatography plate. J. Chromatogr. A, 2010. 1217(23): p. 3711-3715.

123. Krutchinsky, A.N., et al., Thin-layer chromatography laser-desorption of peptides followed by multiphoton ionization time-of-flight mass-spectrometry. J. Mass Spectrom., 1995. 30(2): p. 375-379.

124. Gusev, A.I., et al., Improvement of Signal Reproducibility and Matrix/Comatrix Effects in MALDI Analysis Anal. Chem., 1995. 67(6): p. 1034-1041.

125. Mehl, J.T., A.I. Gusev, and D.M. Hercules, Coupling protocol for thin layer chromatography / matrix-assisted laser desorption ionization. Chromatographia, 1997. 46: p. 358-364.

126. Taki, T. and D. Ishikawa, TLC blotting: application to microscale analysis of lipids and as a new approach to lipid-protein interaction. Anal. Biochem., 1997. 251(2): p. 135-143.

127. Towbin, H., et al., Glycosphingolipid-blotting: an immunological detection procedure after separation by thin layer chromatography. J. Immunol. Methods, 1984. 72(2): p. 471-479.

128. Taki, T., S. Handa, and D. Ishikawa, Blotting of glycolipids and phospholipids from a high-performance thin-layer chromatogram to a polyvinylidene difluoride membrane. Anal. Biochem. , 1994. 221(2): p. 312-316.

129. Taki, T., et al., Direct mass spectrometric analysis of glycosphingolipid transferred to a polyvinylidene difluoride membrane by thin-layer chromatography blotting. Anal. Biochem., 1995. 225(1): p. 24-27.

130. Kasama, T., et al., Microscale analysis of glycosphingolipids by TLC blotting/secondary ion mass spectrometry: a novel blood group A-active glycosphingolipid and changes in glycosphingolipid expression in rat mammary tumour cells with different metastatic potentials. Glycoconj. J., 1996. 13(3): p. 461469.

131. Guittard, J., X.L. Hronowski, and C.E. Costello, Direct matrix-assisted laser desorption/ionization mass spectrometric analysis of glycosphingolipids on thin layer chromatographic plates and transfer membranes. Rapid Commun. Mass Spectrom. , 1999. 13(18): p. 1838-1849.

132. Taki, T., An approach to glycobiology from glycolipidomics: ganglioside molecular scanning in the brains of patients with Alzheimer's disease by TLC-blot/matrix 
assisted laser desorption/ionization-time of flight MS. Biol. Pharm. Bull. , 2012. 35(10): p. 1642-1647.

133. Zaima, N., et al., Selective analysis of lipids by thin-layer chromatography blot matrix-assisted laser desorption/ionization imaging mass spectrometry. J. Oleo. Sci., 2011. 60(2): p. 93-98.

134. Valdes-Gonzales, T., et al., New approach for glyco- and lipidomics--molecular scanning of human brain gangliosides by TLC-Blot and MALDI-QIT-TOF MS. J. Neurochem., 2011. 116 (5): p. 678-683.

135. Vestling, M.M. and C. Fenselau, Surfaces for interfacing protein gel electrophoresis directly with mass spectrometry. Mass Spectrom. Rev., 1995. 14: p. 169-178.

136. Vestling, M.M. and C. Fenselau, Polyvinylidene difluoride membranes as the interface between laser desorption mass spectrometry, gel electrophoresis and in situ proteolysis. Anal. Chem., 1994. 66(2): p. 471-477.

137. Eckerskorn, C., et al., Analysis of proteins by direct-scanning infrared-MALDI mass spectrometry after 2D-PAGE separation and electroblotting. Anal. Chem., 1997. 69 (15): p. 2888-2892.

138. Schleuder, D., F. Hillenkamp, and K. Strupat, IR-MALDI-mass analysis of electroblotted proteins directly from the membrane: comparison of different membranes, application to on-membrane digestion, and protein identification by database searching. Anal. Chem., 1999. 71(15): p. 3238-3247.

139. Schreiner, M., et al., Ultraviolet matrix assisted laser desorption ionization-mass spectrometry of electroblotted proteins. Electrophoresis, 1996. 17(5): p. 954-961.

140. Bunch, J., M.R. Clench, and D.S. Richards, Determination of pharmaceutical compounds in skin by imaging matrix-assisted laser desorption/ionisation mass spectrometry. Rapid Commun. Mass Spectrom. , 2004. 18(24): p. 3051-3060.

141. Vidová, V., et al., Laser desorption-ionization of lipid transfers: tissue mass spectrometry imaging without MALDI matrix. Anal. Chem., 2010. 82(12): p. 49944997.

142. Amstalden van Hove, E.R., et al., A concise review of Imaging Mass Spectrometry. J. Am. Soc. Mass Spectrom., 2011. 22(10): p. 1885-1890.

143. Nesvizhskii, A.I., Protein identification by tandem mass spectrometry and sequence database searching. Methods Mol. Biol. , 2007. 367: p. 87-119.

144. Cottrell, J.S., Protein identification by peptide mass fingerprinting. Pept. Res. , 1994. 7(3): p. 115-124.

145. Mascini, N.E. and R.M.A. Heeren, Protein identification in mass-spectrometry imaging. Trends Anal. Chem., 2012. 40: p. 28-37.

146. Luxembourg, S.L., et al., The molecular scanner in microscope mode. Rapid Commun. Mass Spectrom., 2006. 20(22): p. 3435.

147. Rohner, T.C., D. Staab, and M. Stoeckli, MALDI mass spectrometric imaging of biological tissue sections. Mech. Ageing Dev., 2005. 126 (1): p. 177.

148. Shevchenko, A., et al., In-gel digestion for mass spectrometric characterization of proteins and proteomes. Nat. Protoc., 2006. 1(6): p. 2856-2860.

149. Groseclose, M.R., et al., Identification of proteins directly from tissue: in situ tryptic digestions coupled with imaging mass spectrometry. J. Mass Spectrom., 2007. 42 (2): p. 254-262.

150. Hochstrasser, D.F., et al., A clinical molecular scanner: the Melanie project. MD Comput. , 1991. 8: p. 85-91.

151. Bienvenut, W.V., et al., Toward a Cinical Molecular Scanner for Proteome Research: Parallel Protein Chemical Processing Before and During Western Blot. Anal. Chem. , 1999. 71 (21): p. 4800-4807. 
152. Binz, P.A., et al., The molecular scanner: concept and developments. Curr. Opin. Biotech., 2004. 15(1): p. 17-23.

153. Binz, P.A., et al., A Molecular Scanner to Automate Proteomic Research and to Display Proteome Images. Anal. Chem., 1999. 71 (21): p. 4981-4988.

154. Müller, M., et al., Visualization and analysis of molecular scanner peptide mass spectra. J. Am. Soc. Mass Spectrom., 2002. 13 (3): p. 221-231.

155. Müller, M., et al., Molecular scanner experiment with human plasma: improving protein identification by using intensity distributions of matching peptide masses. Proteomics, 2002. 2(10): p. 1413-1425.

156. Jungmann, J.H. and R.M.A. Heeren, Emerging technologies in mass spectrometry imaging. J. Proteomics 2012. 75(16): p. 5077-5092.

157. Jungmann, J.H. and R.M.A. Heeren, Detection systems for mass spectrometry imaging: A perspective on novel developments with a focus on active pixel detectors. Rapid Commun. Mass Spectrom., 2013. 27(1): p. 1-23.

158. Zhang, X., D.A. Narcisse, and K.K. Murray, On-line single droplet deposition for MALDI mass spectrometry. J. Am. Soc. Mass Spectrom. , 2004. 15(10): p. 14711477.

159. Weidner, S.M. and J. Falkenhagen, LC-MALDI-TOF imaging MS: a new approach in combining chromatography and mass spectrometry of copolymers. Anal. Chem. , 2011. 83(23): p. 9153-9158.

160. Zhang, Z., S. Jiang, and L. Li, Semi-automated liquid chromatography-mass spectrometric imaging platform for enhanced detection and improved data analysis of complex peptides. J. Chromatogr. A, 2013. 1293: p. 44-50.

161. Wang, J., et al., Advancing matrix-assisted laser desorption/ionization-mass spectrometric imaging for capillary electrophoresis analysis of peptides. Anal. Chem. , 2011. 83(9): p. 3462-3469.

162. Hamm, G., et al., Quantitative mass spectrometry imaging of propranolol and olanzapine using tissue extinction calculation as normalization factor. Proteomics, 2012. 30(16): p. 4952-4961.

163. Takai, N., et al., Quantitative analysis of pharmaceutical drug distribution in multiple organs by imaging mass spectrometry. Rapid Commun. Mass Spectrom. , 2012. 26(13): p. 1549-1596.

164. Vismeh, R., et al., Localization and quantification of drugs in animal tissues by use of desorption electrospray ionization mass spectrometry imaging. Anal. Chem., 2012. 84(12): p. 5439-5445.

165. Landgraf, R.R., et al., Considerations for quantification of lipids in nerve tissue using matrix-assisted laser desorption/ionization mass spectrometric imaging. Rapid Commun. Mass Spectrom., 2011. 25(20): p. 3178-3184.

166. Pirman, D.A., et al., Identifying Tissue-Specific Signal Variation in MALDI Mass Spectrometric Imaging by Use of an Internal Standard. Analytical Chemistry, 2013. 85(2): p. 1090-1096.

167. Jaeger, R.J.R., et al., HR-MALDI-MS imaging assisted screening of $\beta$-carboline alkaloids discovered from Mycena metata. J. Nat. Prod., 2013. 76(2): p. 127-134.

168. Grüner, B.M., et al., MALDI Imaging Mass Spectrometry for In Situ Proteomic Analysis of Preneoplastic Lesions in Pancreatic Cancer PLoS ONE, 2012. 7(6): p. e39424.

169. Minerva, L., et al., Linking mass spectrometric imaging and traditional peptidomics: a validation in the obese mouse model. Anal. Chem., 2011. 83(20): p. 7682-7691.

170. Wenk, M.R., The emerging field of lipidomics. Nat Rev Drug Discov, 2005. 4(7): p. 594-610. 
171. Blanksby, S.J. and T.W. Mitchell, Advances in mass spectrometry for lipidomics. Annu Rev Anal Chem (Palo Alto Calif), 2010. 3: p. 433-65.

172. Takats, Z., J.M. Wiseman, and R.G. Cooks, Ambient mass spectrometry using desorption electrospray ionization (DESI): instrumentation, mechanisms and applications in forensics, chemistry, and biology. J Mass Spectrom, 2005. 40(10): p. 1261-75.

173. Hankin, J.A., et al., MALDI mass spectrometric imaging of lipids in rat brain injury models. J Am Soc Mass Spectrom, 2011. 22(6): p. 1014-21.

174. Anderson, D.M., et al., High resolution MALDI imaging mass spectrometry of retinal tissue lipids. J Am Soc Mass Spectrom, 2014. 25(8): p. 1394-403.

175. Skraskova, K. and R.M. Heeren, A review of complementary separation methods and matrix assisted laser desorption ionization-mass spectrometry imaging: lowering sample complexity. J Chromatogr A, 2013. 1319: p. 1-13.

176. Petkovic, M., et al., Detection of individual phospholipids in lipid mixtures by matrix-assisted laser desorption/ionization time-of-flight mass spectrometry: phosphatidylcholine prevents the detection of further species. Anal Biochem, 2001. 289(2): p. 202-16.

177. Zamfir, A., et al., Fully-automated chip-based nanoelectrospray tandem mass spectrometry of gangliosides from human cerebellum. J Am Soc Mass Spectrom, 2004. 15(11): p. 1649-1657.

178. Richards, A.L., et al., Localization and imaging of gangliosides in mouse brain tissue sections by laserspray ionization inlet. J Lipid Res, 2012. 53(7): p. 1390-8.

179. O'Connor, P.B., E. Mirgorodskaya, and C.E. Costello, High pressure matrix-assisted laser desorption/ionization Fourier transform mass spectrometry for minimization of ganglioside fragmentation. J Am Soc Mass Spectrom, 2002. 13(4): p. 402-7.

180. Colsch, B. and A.S. Woods, Localization and imaging of sialylated glycosphingolipids in brain tissue sections by MALDI mass spectrometry. Glycobiology, 2010. 20(6): p. 661-7.

181. Whitehead, S.N., et al., Imaging mass spectrometry detection of gangliosides species in the mouse brain following transient focal cerebral ischemia and long-term recovery. PLoS One, 2011. 6(6): p. e20808.

182. Thomas, A. and P. Chaurand, Advances in tissue section preparation for MALDI imaging MS. Bioanalysis, 2014. 6(7): p. 967-82.

183. Takats, Z., et al., Mass spectrometry sampling under ambient conditions with desorption electrospray ionization. Science, 2004. 306(5695): p. 471-3.

184. Kiss, A. and R.M. Heeren, Size, weight and position: ion mobility spectrometry and imaging MS combined. Anal Bioanal Chem, 2011. 399(8): p. 2623-34.

185. Sladkova, K., J. Houska, and J. Havel, Laser desorption ionization of red phosphorus clusters and their use for mass calibration in time-of-flight mass spectrometry. Rapid Commun Mass Spectrom, 2009. 23(19): p. 3114-8.

186. Juo, C.G., et al., Mass accuracy improvement of reversed-phase liquid chromatography/electrospray ionization mass spectrometry based urinary metabolomic analysis by post-run calibration using sodium formate cluster ions. Rapid Commun Mass Spectrom, 2014. 28(16): p. 1813-20.

187. Ritorto, M.S. and J. Borlak, A simple and reliable protocol for mouse serum proteome profiling studies by use of two-dimensional electrophoresis and MALDI TOF/TOF mass spectrometry. Proteome Sci, 2008. 6: p. 25.

188. Neubert, H., et al., MALDI post-source decay and LIFT-TOF/TOF investigation of $\alpha$ cyano-4-hydroxycinnamic acid cluster interferences. Journal of the American Society for Mass Spectrometry, 2004. 15(3): p. 336-343. 
189. Smirnov, I.P., et al., Suppression of alpha-cyano-4-hydroxycinnamic acid matrix clusters and reduction of chemical noise in MALDI-TOF mass spectrometry. Anal Chem, 2004. 76(10): p. 2958-65.

190. Krutchinsky, A.N. and B.T. Chait, On the nature of the chemical noise in MALDI mass spectra. J Am Soc Mass Spectrom, 2002. 13(2): p. 129-34.

191. Fuchs, B., Mass spectrometry and inflammation--MS methods to study oxidation and enzyme-induced changes of phospholipids. Anal Bioanal Chem, 2014. 406(5): $\mathrm{p}$. 1291-306.

192. Wiseman, J.M. and J.B. Li, Elution, partial separation, and identification of lipids directly from tissue slices on planar chromatography media by desorption electrospray ionization mass spectrometry. Anal Chem, 2010. 82(21): p. 8866-74.

193. O'Brien, J.P. and J.S. Brodbelt, Structural characterization of gangliosides and glycolipids via ultraviolet photodissociation mass spectrometry. Anal Chem, 2013. 85(21): p. 10399-407.

194. Rožman, M., et al., Database and data analysis application for structural characterization of gangliosides and sulfated glycosphingolipids by negative ion mass spectrometry. Carbohydrate Research, 2014. 400(0): p. 1-8.

195. Vajn, K., et al., Differential distribution of major brain gangliosides in the adult mouse central nervous system. PLoS One, 2013. 8(9): p. e75720.

196. http://www.brain-map.org/.

197. Walkley, S.U., Secondary accumulation of gangliosides in lysosomal storage disorders. Semin Cell Dev Biol, 2004. 15(4): p. 433-44.

198. Manicke, N.E., et al., Imaging of lipids in atheroma by desorption electrospray ionization mass spectrometry. Anal Chem, 2009. 81(21): p. 8702-7.

199. Ellis, S.R., et al., Imaging of human lens lipids by desorption electrospray ionization mass spectrometry. J Am Soc Mass Spectrom, 2010. 21(12): p. 2095-104.

200. Jackson, S.N., et al., MALDI-Ion Mobility Mass Spectrometry of Lipids in Negative Ion Mode. Anal Methods, 2014. 6(14): p. 5001-5007.

201. Ruh, H., et al., MALDI imaging MS reveals candidate lipid markers of polycystic kidney disease. J Lipid Res, 2013. 54(10): p. 2785-94.

202. Murphy, R.C., et al., MALDI Imaging of Lipids after Matrix Sublimation/Deposition. Biochimica et biophysica acta, 2011. 1811(11): p. 970-975.

203. Pol, J., et al., Molecular mass spectrometry imaging in biomedical and life science research. Histochem Cell Biol, 2010. 134(5): p. 423-43.

204. Chughtai, S., et al., A multimodal mass spectrometry imaging approach for the study of musculoskeletal tissues. Int. J. Mass Spectrom., 2012. 325-327(0): p. 150-160.

205. Walch, A., et al., MALDI imaging mass spectrometry for direct tissue analysis: a new frontier for molecular histology. Histochem Cell Biol, 2008. 130(3): p. 421-34.

206. Fuoco, E.R., et al., Surface analysis studies of yield enhancement in secondary ion mass spectrometry by polyatomic projectiles. J. Phys. Chem. B, 2001. 105: p. 39503956.

207. Sheraz née Rabbani, S., et al., Enhancing secondary ion yields in time of flightsecondary ion mass spectrometry using water cluster primary beams. Anal. Chem., 2013. 85(12): p. 5654-5658.

208. Brunelle, A., D. Touboul, and O. Laprévote, Biological tissue imaging with time-offlight secondary ion mass spectrometry and cluster ion sources. J. Mass Spectrom., 2005. 40(8): p. 985-999.

209. Goto-Inoue, N., et al., Imaging mass spectrometry for lipidomics. Biochim. Biophys. Acta, 2011. 1811(11): p. 961-969.

210. Murphy, R.C., et al., MALDI imaging of lipids after matrix sublimation/deposition. Biochim. Biophys. Acta, Mol. Cell Biol. Lipids, 2011. 1811(11): p. 970-975. 
211. Todd, P.J., et al., Organic ion imaging of biological tissue with secondary ion mass spectrometry and matrix-assisted laser desorption/ionization. J. Mass Spectrom., 2001. 36(4): p. 355-369.

212. van Hove, E.R.A., et al., Multimodal mass spectrometric imaging of small molecules reveals distinct spatio-molecular signatures in differentially metastatic breast tumor models. Cancer Res., 2010. 70(22): p. 9012-9021.

213. Chughtai, K., et al., Fiducial markers for combined 3-dimensional mass spectrometric and optical tissue imaging. Anal. Chem., 2012. 84(4): p. 1817-1823.

214. Parker, C.C., et al., Magnetic resonance imaging in the radiation treatment planning of localized prostate cancer using intra-prostatic fiducial markers for computed tomography co-registration. Radiother. Oncol., 2003. 66(2): p. 217-224.

215. Unlu, M.Z., et al., Computerized method for nonrigid MR-to-PET breast-image registration. Comput. Biol. Med., 2010. 40: p. 37-53.

216. Lim, Y.K., et al., Microscopic gold particle-based fiducial markers for proton therapy of prostate cancer. Int. J. Radiat. Oncol., 2009. 74(5): p. 1609-1616.

217. Klein, O., et al., MALDI imaging mass spectrometry: Discrimination of pathophysiological regions in traumatized skeletal muscle by characteristic peptide signatures. Proteomics, 2014. 14(20): p. 2249-60.

218. Hanrieder, J., et al., High resolution metabolite imaging in the hippocampus following neonatal exposure to the environmental toxin BMAA using ToF-SIMS. ACS Chem. Neurosci., 2014. 5(7): p. 568-75.

219. Jones, E.A., et al., Imaging mass spectrometry to visualize biomolecule distributions in mouse brain tissue following hemispheric cortical spreading depression. $\mathrm{J}$ Proteomics, 2012. 75(16): p. 5027-35.

220. McCombie, G., et al., Spatial and spectral correlations in MALDI mass spectrometry images by clustering and multivariate analysis. Anal Chem, 2005. 77(19): p. 611824.

221. Abdelmoula, W.M., et al., Automatic registration of mass spectrometry imaging data sets to the Allen brain atlas. Anal. Chem., 2014. 86(8): p. 3947-3954.

222. Matusch, A., et al., Combined elemental and biomolecular mass spectrometry imaging for probing the inventory of tissue at a micrometer scale. Anal Chem, 2012. 84(7): p. 3170-8.

223. Abdelmoula, W.M., et al., Automatic Generic Registration of Mass Spectrometry Imaging Data to Histology using Nonlinear Stochastic Embedding. Anal. Chem., 2014. 86(18): p. 9204-9211.

224. van der Maaten, L. and G. Hinton, Visualizing data using t-SNE. J. Mach. Learn. Res., 2008. 85(9): p. 2579-2605.

225. Lein, E.S., et al., Genome-wide atlas of gene expression in the adult mouse brain. Nature, 2007. 445(7124): p. 168-176.

226. Verbeeck, N., et al., Automated anatomical interpretation of ion distributions in tissue: linking imaging mass spectrometry to curated atlases. Anal. Chem., 2014. 86(18): p. 8974-82.

227. Huyghe, S., et al., Peroxisomal multifunctional protein-2: the enzyme, the patients and the knockout mouse model. Biochim Biophys Acta, 2006. 1761(9): p. 973-94.

228. Verheijden, S., et al., Peroxisomal multifunctional protein-2 deficiency causes neuroinflammation and degeneration of Purkinje cells independent of very long chain fatty acid accumulation. Neurobiol Dis, 2013. 58: p. 258-269.

229. Huyghe, S., et al., Peroxisomal multifunctional protein-2 deficiency causes motor deficits and glial lesions in the adult central nervous system. Am J Pathol, 2006. 168(4): p. 1321-34. 
230. Chaurand, P., et al., Integrating histology and imaging mass spectrometry. Anal. Chem., 2004. 76(4): p. 1145-1155.

231. Eijkel, G.B., et al., Correlating MALDI and SIMS imaging mass spectrometric datasets of biological tissue surfaces. Surf Inerface Anal, 2009. 41(8): p. 675-685.

232. Gerber, F., et al., Multiorder correction algorithms to remove image distortions from mass spectrometry imaging data sets. Anal. Chem., 2013. 85(21): p. 10249-54.

233. http://homepage.tudelft.nl/19j49/t-SNE.html.

234. Verheijden, S., et al., Central nervous system pathology in MFP2 deficiency: insights from general and conditional knockout mouse models. Biochimie, 2014. 98: p. 11926.

235. Dinkel, K., et al., Stiff-man syndrome: identification of 17 $\beta$-hydroxysteroid dehydrogenase type 4 as a novel 80-kDa antineuronal antigen. Journal of Neuroimmunology, 2002. 130(1-2): p. 184-193.

236. Borges, L.F., et al., Selective extraction of small and large molecules from the cerebrospinal fluid by Purkinje neurons. Science, 1985. 228(4697): p. 346-348.

237. da Silva, R.J.G., I.C.L. Pereira, and J.R.M. Oliveira, Analysis of Gene Expression Pattern and Neuroanatomical Correlates for SLC20A2 (PiT-2) Shows a Molecular Network with Potential Impact in Idiopathic Basal Ganglia Calcification ("Fahr's Disease”). Journal of Molecular Neuroscience, 2013. 50(2): p. 280-283.

238. http://www.uniprot.org/.

239. Ashibe, B., et al., Dual subcellular localization in the endoplasmic reticulum and peroxisomes and a vital role in protecting against oxidative stress of fatty aldehyde dehydrogenase are achieved by alternative splicing. J. Biol. Chem., 2007. 282(28): p. 20763-73.

240. Postawa, Z., Sputtering simulations of organic overlayers on metal substrates by monoatomic and clusters projectiles. Applied Surface Science, 2004. 231-232: p. 2228.

241. Eberlin, L.S., et al., Nondestructive, histologically compatible tissue imaging by desorption electrospray ionization mass spectrometry. Chembiochem, 2011. 12(14): p. 2129-32.

242. Dill, A.L., et al., Data quality in tissue analysis using desorption electrospray ionization. Anal Bioanal Chem, 2011. 401(6): p. 1949-61.

243. Fischer, C.R., O. Ruebel, and B.P. Bowen, An accessible, scalable ecosystem for enabling and sharing diverse mass spectrometry imaging analyses. Arch Biochem Biophys, 2015.

244. McDonnell, L.A., et al., Discussion point: reporting guidelines for mass spectrometry imaging. Anal Bioanal Chem, 2015. 407(8): p. 2035-45.

245. https://www.humanbrainproject.eu/.

246. http://www.braininitiative.nih.gov/.

247. Sharon, R., et al., The formation of highly soluble oligomers of alpha-synuclein is regulated by fatty acids and enhanced in Parkinson's disease. Neuron, 2003. 37(4): p. 583-95. 


\subsection{Publications}

\section{This thesis is based on the following publications}

Karolina Škrášková and Ron M.A. Heeren: A review of complementary separation methods and Matrix assisted laser desorption ionization-mass spectrometry imaging: lowering sample complexity. J. Chromatogr. A 1319, 1-13 (2013). (Chapter 2)

Karolina Škrášková, Emmanuelle Claude, Emrys A. Jones, Mark Towers, Shane R. Ellis, and Ron M. A. Heeren: Enhanced capabilities for imaging gangliosides in murine brain with desorption electrospray ionization mass spectrometry coupled to ion mobility separation. Manuscript submitted to Methods. (Chapter 3)

Nina Ogrinc Potočnik^, Karolina Škrášková^^, Bryn Flinders, Primož Pelicon, and Ron M. A. Heeren: Gold Sputtered Fiducial Markers for Combined SIMS and MALDI Imaging of Tissue Samples. Anal. Chem. 86, 6781-6785 (2014). ^Equal contribution. (Chapter 4)

Karolina Škrášková, Artem Khmelinskii, Walid M. Abdelmoula, Stephanie De Munter, Jouke Dijkstra, Myriam Baes, and Ron M. A. Heeren: Precise anatomical localization of accumulated lipids in Mfp2 deficient murine brains through automated registration of SIMS images to the Allen Brain Atlas. J. Amer. Soc. Mass Spectrom. 26, 948-957 (2015). (Chapter 5)

\section{Other publications}

Walid M. Abdelmoula, Karolina Škrášková, Benjamin Balluff, Ricardo J. Carreira, Else A. Tolner, Boudewijn F.P. Lelieveldt, Laurens van der Maaten, Hans Morreau, Arn M. J.M. van den Maagdenberg, Ron M. A. Heeren, Liam A. McDonnell, and Jouke Dijkstra: Automatic Generic Registration of Mass Spectrometry Imaging Data to Histology using Nonlinear Stochastic Embedding. Anal. Chem. 86, 9204-9211 (2014).

Anna Bodzon-Kulakowska, Karolina Škrášková, Kamila Chughtai, Gert Eijkel, Marta Marszalek, Jolanta Kotlińska, Ron M. A. Heeren, and Piotr Suder: Lipidomic changes after morphine administration: A mass spectrometry imaging study. Manuscript in preparation.

Karolina Škrášková, Lúcia H.M.L.M. Santos, Dalibor Šatínský, Angelina Peňa, Maria Conceicao B.S.M. Montenegro, Petr Solich, and Lucie Nováková: Fast and sensitive UHPLC methods with fluorescence and tandem mass spectrometry detection for the determination of tetracycline antibiotics in surface waters. J. Chromatogr. B 927 201-208 (2013). 



\section{Acknowledgements}

Finally, my PhD Odyssey reached its end! Starting it in the Czech town of Hradec Králové, continuing through Amsterdam and concluding in Maastricht: this sail was especially demanding. But it was also very enjoyable. Mostly thanks to the sailors I met on the way. Some of them asked me thoughtful questions, others gave me wise answers, but all of them taught me new things and enabled me to reach the harbour eventually. It seems unfair to name only some of them. Yet, to thank to them all is beyond one's comprehension. I feel humbled and grateful for all of you being part of my journey, dear Colleagues, Collaborators, Friends, Family members, Advisors \& Good Fairies. I would like to take the opportunity of this space to particularly acknowledge those people without whose navigation I would have been wrecked on the $\mathrm{PhD}$ sea.

\section{Thank you,}

Ron, for taking me onboard! I have learned much more than what I had hoped for! Thank you for trusting me. Thank you for being my wind \& sail: it would not have been possible to move forward without you. Thank you also, at last, for moving us to Maastricht - my legs have never been so strong, I love cycling in Limburg!

Shane, for making time for me when I needed it the most. Your knowledge of mass spectrometry and of what goes beyond amazes me and I am thankful that you shared some of it with me!

Rosko, for going through the challenges of this adventure with me. Thank you for being my compass: the constant reminder of what really matters in life. Thank you for your patience, kindness, generosity and inspiration. I feel grateful for your decision to elucidate oak aromas using mass spectrometry. A deep bow to your courageous spirit!

Brenda Bakker, Benjamin Balluff, Florian Barré, Anne Bruinen, Ronald Buijs, Jo Cappell, Berta Cillero Pastor, Kamila Chugthai, Bryn Flinders, Marc Dursmaa, Gert Eijkel, Shane Ellis, Frans Giskes, Bob Hommersom, Lennart Huizing, Mark Jansen, Guzel Kireeva, Andras Kiss, Ireen Kleijnen, Ivo Klinkert, Pieter Kooijman, Lieke Lamont, Florian Marty, Nadine Mascini, Tiff Porta, Nina Ogrinc-Potočnik, Keely Pierzchalski, Klára Ščupáková, Livia Smits, Julia \& Don Smith, Sarfaraz Syed. Thank you for being such an amazing team! Thank you for helping me throughout this journey from its very beginning (when I was getting acquainted with MS imaging and was wondering what "PCA" stands for) till its very end (when providing me with feedback during my defence preparations). A special Thank you to all of you who moved from AMOLF to M4I - I am proud of us making this happen! 
Berta \& Felipe, for being my paranypmhs. Not only on the day of the defence!

Emmanuelle, for your help with the DESI project. Thank you for sharing your expertise with me; I have learned a lot from you!

Artem, Walid \& Liam. I had lots of fun working on the Allen Brain Atlas project! Thank you for making it possible.

Myriam \& Stephanie, for adding the taste of biology to my $\mathrm{PhD}$ research.

professor Solich for providing me with maps initially and for letting me change the ships!

Annchen, Janine \& Monica, for your friendship and support. You have dosed me with your feistiness and courage and your love of the ironman world. My life will never be the same!

Bobina for being my moral support. I am grateful for the invention of Skype!

Mum \& Dad, for being my anchor: keeping my boat in place when the $\mathrm{PhD}$ sea became too wild at times. Thank you for supporting me when I do things I love even if they involve the most distant places of the world! // Děkuji z celého srdce svým rodičům, Radce a Jirkovi. Pokud na plavbě skrz PhD Ron byl mým větrem a mou plachtou a Rosko mým kompasem, pak Vy jste byli mojí kotvou. Ta mě držela na místě, když se $\mathrm{PhD}$ moře někdy príliš rozbouřilo. Děkuji Vám také za to, že mě podporujete v dělání věcí, jež miluji - i když je to na těch nejvzdálenějších místech světa!

At last, I would like to remember my dear friend Radek. His life attitude is my light in the times of darkness. I miss you deeply. 
And Thank you, // A Děkuji, ...

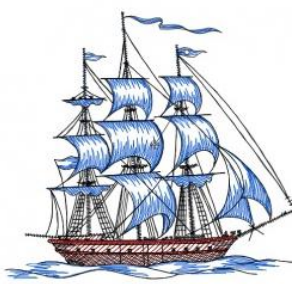

"As you sail thru life, don't avoid rough waters because calm waters won't make a skilful sailor." 



\section{Curriculum Vitae}

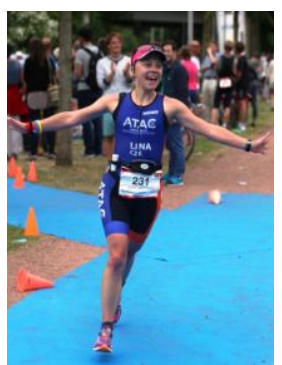

I was born in Plzeň, the Czech Republic on $6^{\text {th }}$ January 1987. graduated from a grammar school in my hometown in 2006 and in the same year started the longed-for study of pharmacy at the Faculty of Pharmacy at the Charles University, Hradec Králové, Czech Republic. In the final year of the undergraduate studies, I spent four months working on my master thesis in the Arrhenius laboratoriet at the Stockholm University. This was my first hand-on experience with mass spectrometry, and the lab work strengthened my passion for research and science.

After working in a community pharmacy for several months following the graduation in 2011, I have entered a doctoral study programme at the Faculty of Pharmacy at the Charles University at the Department of Analytical Chemistry. My $\mathrm{PhD}$ research revolved around liquid chromatography and mass spectrometry (LCMS), which we used for a development of methods for quantification of pharmaceuticals in various matrices, such as surface waters, biological fluids or food. My desire for learning and wanting to know ever more, led me to a 6-month internship at $A M O L F$, Amsterdam, The Netherlands, where I worked under a supervision of professor Ron Heeren on projects using mass spectrometry imaging (MSI). To link the knowledge on pharmacy, LCMS quantification and MSI, I underwent a short internship at the Novartis Institutes for BioMedical Research, Basel, Switzerland at the end of 2012, where I explored quantification approaches for MSI.

By the time my internship at AMOLF was drawing to its end, I was offered a permanent $\mathrm{PhD}$ position ibidem, which I accepted. I have joined Ron Heeren's Biomedical Imaging Mass Spectrometry group as an official $\mathrm{PhD}$ researcher in March 2013 and started the work on lipid imaging. At the beginning of 2015, the whole group relocated to new facilities in Maastricht within the newly found Maastricht Multimodal Molecular Imaging Institute (M4I). My research in both, AMOLF and M4I, was aimed at the development of new approaches for multimodal imaging of lipids in the murine brain. The results my work are summarized in this thesis. 
